- Supporting Information -

\title{
Nucleophilicities of Nitroalkyl Anions
}

\author{
Thorsten Bug, Tadeusz Lemek, and Herbert Mayr ${ }^{*}$ \\ Department Chemie und Biochemie der Ludwig-Maximilians-Universität München, \\ Butenandtstr. 5-13 (Haus F), D-81377 München, Germany
}

Contents:

Page

1 Preparative Reactions $\quad$ S 2

$\begin{array}{ll}1.1 \text { General } & \text { S } 2\end{array}$

1.2 Synthesis of the aryl substituted $\mathrm{CH}$ acids (1d-g)-H $\quad$ S 2

1.3 Reactions of nitroalkyl anions with benzhydrylium salts in water $\quad$ S 5

1.4 Reactions of the nitroethane anion $\left(\mathbf{1 b}-\mathrm{NBu}_{4}{ }^{+}\right)$with quinone methides $\mathbf{2} \mathbf{f}-\mathbf{h} \quad \mathrm{S} 11$

2 Kinetic Investigations $\quad$ S 13

2.1 Kinetic investigations of nitroalkyl anions in water $\quad$ S 13

2.1.1 Remarks $\quad$ S 13

2.1.2 Calculation of the nitroalkyl anion concentration $\left[\mathrm{C}^{-}\right]$in aqueous solutions $\quad \mathrm{S} 14$

2.1.3 Individual kinetic runs in water (Tables S1-S26) $\quad$ S 15

2.2 Kinetic investigations of nitroalkyl anions in DMSO S 41

2.2.1 Remarks $\quad$ S 41

2.2.2 Individual kinetic runs in DMSO (Tables S27-S49) $\quad$ S 42

3 References $\quad$ S 65 


\section{Preparative Reactions}

\subsection{General}

${ }^{1} \mathrm{H}$ NMR and ${ }^{13} \mathrm{C}$ NMR spectra were recorded with a Bruker ARX $300(300 \mathrm{MHz}, 75.5 \mathrm{MHz})$ or Bruker AMX $400(400 \mathrm{MHz}, 100.1 \mathrm{MHz})$. Chemical shifts are reported on the $\delta$ scale relative to tetramethylsilane $\left(\delta_{\mathrm{H}}=0.00\right), \mathrm{CDCl}_{3}\left(\delta_{\mathrm{C}}=77.00\right)$, or $\mathrm{C}_{6} \mathrm{D}_{6}\left(\delta_{\mathrm{C}}=128.00\right)$ as internal standards. Abbreviations used are s (singlet), $\mathrm{d}$ (dublet), $\mathrm{t}$ (triplet), q (quartet) and $\mathrm{m}$ (multiplet). Infrared spectra were recorded with a Perkin-Elmer FT-IR 1000 spectrophotometer. Mass spectra were measured with a Finnigan MAT 95 Q. Microanalyses were carried out by the Mikroanalytisches Labor des Departments Chemie der LMU München. Melting points were determined on a Büchi B-540 and are uncorrected.

\subsection{Synthesis of the aryl substituted $\mathrm{CH}$ acids (1d-g)-H}

During the syntheses of the $\mathrm{CH}$ acids $(\mathbf{1 d}-\mathbf{g})-\mathbf{H}$ from the corresponding aryl bromides, the reaction mixtures have to be protected from sun light to avoid the formation of side products.

Phenylnitromethane (1d-H). Benzyl bromide (10.4 mL, $87.6 \mathrm{mmol})$ was added to a stirred and precooled $\left(-20{ }^{\circ} \mathrm{C}\right)$ solution of $\mathrm{NaNO}_{2}(10.3 \mathrm{~g}, 149 \mathrm{mmol})$ and urea $(11.6 \mathrm{~g}, 193 \mathrm{mmol})$ in DMF $(180 \mathrm{~mL})$. After $5 \mathrm{~h}$ at $-20^{\circ} \mathrm{C}$, the solution was poured into ice-water $(400 \mathrm{~mL})$ and extracted with ether $(3 \times 100 \mathrm{~mL})$. The combined organic extracts were washed with water $(3 \times 100 \mathrm{~mL})$, dried over $\mathrm{MgSO}_{4}$, and the solvent was evaporated. The oily residue was distilled $\left(68-72{ }^{\circ} \mathrm{C} / 0.5 \mathrm{mbar}\right.$; ref S1: $\left.77-79{ }^{\circ} \mathrm{C} / 1 \mathrm{~mm}\right)$ to yield $\mathbf{1 d - H}\left(3.72 \mathrm{~g}, 31 \%\right.$ ) as an yellow oil. $\mathrm{n}_{D}^{20}=1.530$ (ref S1: 1.5315). ${ }^{1} \mathrm{H}$ NMR $\left(\mathrm{CDCl}_{3}, 300 \mathrm{MHz}\right): \delta=5.42\left(\mathrm{~s}, 2 \mathrm{H}, \mathrm{CH}_{2} \mathrm{NO}_{2}\right), 7.43$ (br s, $\left.5 \mathrm{H}\right) ;{ }^{[\mathrm{S} 2]}{ }^{13} \mathrm{C} \mathrm{NMR}$ $\left(\mathrm{CDCl}_{3}, 75.5 \mathrm{MHz}\right): \delta=79.98$ (t, $\left.\mathrm{CH}_{2} \mathrm{NO}_{2}\right), 129.03$ (d), 129.69 (s), 129.92 (d), 129.93 (d) ${ }^{[\mathrm{S} 2]} \mathrm{MS}$ $(\mathrm{EI}, 70 \mathrm{eV}): m / z(\%)=92(10), 91$ (100), 65 (14) ; $^{[\mathrm{S} 2]} \mathrm{IR}(\mathrm{Film}): \widetilde{v}=3068,3036,2916,1555$, $1498,1456,1375,1313,1191,718 \mathrm{~cm}^{-1}{ }^{[\mathrm{S} 2]}$

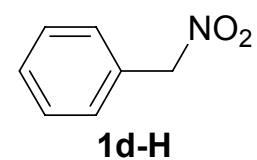

(4-Methylphenyl)nitromethane (1e-H). 4-Methyl-benzyl bromide (15.0 g, $81.0 \mathrm{mmol})$ was added to a stirred and precooled $\left(-20{ }^{\circ} \mathrm{C}\right)$ solution of $\mathrm{NaNO}_{2}(8.40 \mathrm{~g}, 122 \mathrm{mmol})$ and urea $(9.70 \mathrm{~g}, 162$ $\mathrm{mmol})$ in DMF $(150 \mathrm{~mL})$. After $7 \mathrm{~h}$ at $-20^{\circ} \mathrm{C}$, the solution was poured into ice-water $(300 \mathrm{~mL})$ 
and extracted with ether $(3 \times 80 \mathrm{~mL})$. The combined organic extracts were washed with water $(3 \times$ $100 \mathrm{~mL}$ ), dried over $\mathrm{MgSO}_{4}$, and the solvent was evaporated. The oily residue was distilled at 78$82{ }^{\circ} \mathrm{C} / 0.7 \mathrm{mbar}$ (ref S1: $99^{\circ} \mathrm{C} / 3 \mathrm{~mm}$ ) to yield 1e-H $(4.10 \mathrm{~g}, 34 \%)$ as a pale yellow liquid. ${ }^{1} \mathrm{H}$ NMR $\left(\mathrm{CDCl}_{3}, 300 \mathrm{MHz}\right): \delta=2.37(\mathrm{~s}, 3 \mathrm{H}), 5.39\left(\mathrm{~s}, 2 \mathrm{H}, \mathrm{CH}_{2} \mathrm{NO}_{2}\right), 7.22,7.33\left(2 \mathrm{~d}, \mathrm{AA}^{\prime} \mathrm{BB}^{\prime}\right.$ system with $\left.J_{\mathrm{AB}}=8.1 \mathrm{~Hz}, 2 \times 2 \mathrm{H}\right) ;{ }^{[\mathrm{S} 3]}{ }^{13} \mathrm{C} \mathrm{NMR}\left(\mathrm{CDCl}_{3}, 75.5 \mathrm{MHz}\right): \delta=21.2$ (q), $79.8(\mathrm{t}$, $\mathrm{CH}_{2} \mathrm{NO}_{2}$ ), 126.8 (s), 129.7 (d), 129.9 (d), 140.1 (s); MS (EI, $\left.70 \mathrm{eV}\right): m / z$ (\%) = 105 (100), 79 (11), 77 (14); IR (Film): $\widetilde{v}=3032,2923,1617,1554,1518,1432,1372,1312,1186,778,723 \mathrm{~cm}^{-1}$. [S3] Anal. Calcd for $\mathrm{C}_{8} \mathrm{H}_{9} \mathrm{NO}_{2}$ (151.16): C 63.56, H 6.00, N 9.27. Found: C 63.93, H 6.35, N 8.97.

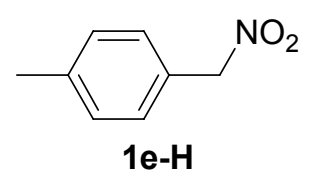

(4-Nitrophenyl)nitromethane (1f-H). 4-Nitro-benzyl bromide (25.0 g, $116 \mathrm{mmol})$ was added to a stirred and precooled $\left(-20^{\circ} \mathrm{C}\right)$ solution of $\mathrm{NaNO}_{2}(12.0 \mathrm{~g}, 174 \mathrm{mmol})$ and urea $(14.0 \mathrm{~g}, 232 \mathrm{mmol})$ in DMF $(200 \mathrm{~mL})$. After $5 \mathrm{~h}$ at $-20{ }^{\circ} \mathrm{C}$, the solution was poured into ice-water $(200 \mathrm{~mL})$ and extracted with ether $(3 \times 80 \mathrm{~mL})$. The combined organic extracts were washed with water $(3 \times 150$ $\mathrm{mL}$ ), dried over $\mathrm{MgSO}_{4}$, and the solvent was evaporated. The crude product was purified by column chromatography ( $n$-pentane $/ \mathrm{Et}_{2} \mathrm{O}(5: 1), R_{\mathrm{f}}=0.33\left(n\right.$-pentan $\left./ \mathrm{Et}_{2} \mathrm{O}=1: 1\right)$ ) to yield $\mathbf{1 f}-\mathbf{H}$ $(5.20 \mathrm{~g}, 25 \%)$ as a pale yellow solid. M.p. $84-86{ }^{\circ} \mathrm{C}$ (ref S4: $\left.89-91{ }^{\circ} \mathrm{C}\right) .{ }^{1} \mathrm{H}$ NMR $\left(\mathrm{CDCl}_{3}, 300\right.$ MHz): $\delta=5.57\left(\mathrm{~s}, 2 \mathrm{H}, \mathrm{CH}_{2} \mathrm{NO}_{2}\right), 7.67(\mathrm{~d}, J=8.7 \mathrm{~Hz}, 2 \mathrm{H}), 8.31(\mathrm{~d}, J=8.7 \mathrm{~Hz}, 2 \mathrm{H}) ;{ }^{13} \mathrm{C} \mathrm{NMR}$ $\left(\mathrm{CDCl}_{3}, 75.5 \mathrm{MHz}\right): \delta=78.7$ (t, $\left.\mathrm{CH}_{2} \mathrm{NO}_{2}\right), 124.2$ (d), 131.1 (d), 135.7 (s), 148.9 (s); MS (EI, 70 $\mathrm{eV}): m / z(\%)=166(7), 137(9), 136(100), 106(14), 89(13)$; IR (KBr): $\widetilde{v}=1610,1548,1528$, $1352,1313,716 \mathrm{~cm}^{-1}$; UV/Vis $\left(\mathrm{CH}_{3} \mathrm{CN}\right): \lambda_{\max }(\log \varepsilon)=261(4.01)$.

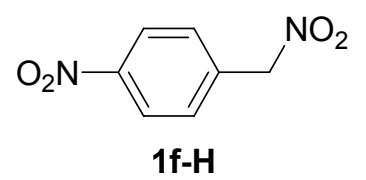

(3-Nitrophenyl)nitromethane (1g-H). $\mathrm{NaNO}_{2}(2.40 \mathrm{~g}, 34.7 \mathrm{mmol})$ and urea (2.80 g, $\left.46.3 \mathrm{mmol}\right)$ were dissolved in DMF $(30 \mathrm{~mL})$ and cooled to $-20^{\circ} \mathrm{C}$. Then a solution of 3-nitro-benzyl bromide $(5.00 \mathrm{~g}, 23.1 \mathrm{mmol})$ in DMF $(20 \mathrm{~mL})$ was slowly added $(15 \mathrm{~min})$ and stirred for $8 \mathrm{~h}$ at $-20^{\circ} \mathrm{C}$. The reaction mixture was added to water $(200 \mathrm{~mL})$, and the aqueous layer was extracted with ether $(3 \times$ $40 \mathrm{~mL})$. The combined organic layers were washed with water $(3 \times 50 \mathrm{~mL})$, dried over $\mathrm{Na}_{2} \mathrm{SO}_{4}$, and the solvent was evaporated. The crude product was stirred with $n$-pentane $(3 \times 15 \mathrm{~mL})$ to dissolve impurities. Recrystallization of the solid residue yielded $\mathbf{1 g}-\mathbf{H}(1.70 \mathrm{~g}, 40 \%)$ as yellow 
crystals. M.p. $91-93{ }^{\circ} \mathrm{C}\left(\right.$ lit. $\left.^{23}: 91-92{ }^{\circ} \mathrm{C}\right) .{ }^{1} \mathrm{H}$ NMR $\left(\mathrm{CDCl}_{3}, 300 \mathrm{MHz}\right): \delta=5.56(\mathrm{~s}, 2 \mathrm{H}$, $\left.\mathrm{CH}_{2} \mathrm{NO}_{2}\right), 7.67(\mathrm{t}, J=7.8 \mathrm{~Hz}, 1 \mathrm{H}), 7.82(\mathrm{~d}, J=7.7 \mathrm{~Hz}, 1 \mathrm{H}), 8.29-8.40(\mathrm{~m}, 2 \mathrm{H}) ;{ }^{13} \mathrm{C} \mathrm{NMR}$ $\left(\mathrm{CDCl}_{3}, 75.5 \mathrm{MHz}\right): \delta=78.6$ (t, $\left.\mathrm{CH}_{2} \mathrm{NO}_{2}\right), 125.0$ (d), 125.2 (d), 130.3 (d), 131.1 (s), 136.0 (d), 148.5 (s); MS (EI, $70 \mathrm{eV}): m / z(\%)=166$ (1), 137 (8), 136 (100), 90 (50), 89 (31); IR (KBr): $\widetilde{v}=$ 3089, 2965, 2915, 1622, 1546, 1523, 1378, 1352, 1318, 1102, 816, $717 \mathrm{~cm}^{-1}$. Anal. Calcd for $\mathrm{C}_{7} \mathrm{H}_{6} \mathrm{~N}_{2} \mathrm{O}_{4}$ (182.13): C 46.16, H 3.32, N 15.38. Found: C 46.50, H 3.18, N 15.20.

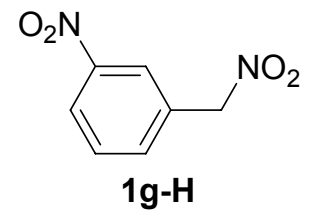




\subsection{Reactions of nitroalkyl anions with benzhydrylium salts in water}

Nitroalkanes $(\mathbf{1 a}-\mathbf{g})-\mathrm{H}(0.5-5.0 \mathrm{mmol})$ were dissolved in $1 \mathrm{~mL}$ of $0.5 \mathrm{M}$ aqeous $\mathrm{KOH}$. Then acetonitrile $(0.5-1.5 \mathrm{~mL})$ and the solid benzhydrylium tetrafluoroborates $(0.5 \mathrm{mmol})$ were added. The reaction mixture was stirred at room temperature (ca. $20{ }^{\circ} \mathrm{C}$ ) until the color of the benzhydrylium ion had faded and the product separated from the reaction mixture (either as a precipitate or as an oily layer).

The conditions of the reactions were not optimized for high yields.

\section{1,1-Bis(4-dimethylaminophenyl)-2-nitroethane (3a)}

$260 \mu \mathrm{L}(4.81 \mathrm{mmol})$ nitromethane (19-H)

$1.00 \mathrm{~mL}(0.50 \mathrm{mmol}) 0.5 \mathrm{M} \mathrm{KOH}$

$170 \mathrm{mg}(0.50 \mathrm{mmol}) \mathbf{2 a}$

$0.50 \mathrm{~mL}$ acetonitrile

After $30 \mathrm{~min}$, a precipitate had formed that was collected by filtration, washed with water $(3 \times 15$ $\mathrm{mL}$ ), and dried in vacuo to yield 3a (135 mg, $86 \%$ ) as a colorless solid. Mp. $124-127{ }^{\circ} \mathrm{C} .{ }^{1} \mathrm{H}$ $\operatorname{NMR}\left(\mathrm{CDCl}_{3}, 300 \mathrm{MHz}\right): \delta=2.90\left(\mathrm{~s}, 12 \mathrm{H}, \mathrm{NMe}_{2}\right), 4.71\left(\mathrm{t}, J=8.2 \mathrm{~Hz}, 1 \mathrm{H}, \mathrm{Ar}_{2} \mathrm{CH}\right), 4.88(\mathrm{~d}, J=$ $\left.8.1 \mathrm{~Hz}, 2 \mathrm{H}, \mathrm{CH}_{2} \mathrm{NO}_{2}\right), 6.66,7.08\left(2 \mathrm{~d}, \mathrm{AA}^{\prime} \mathrm{BB}^{\prime}\right.$ system with $\left.J_{\mathrm{AB}}=8.7 \mathrm{~Hz}, 2 \times 4 \mathrm{H}, \mathrm{ArH}\right) ;{ }^{13} \mathrm{C}$ $\operatorname{NMR}\left(\mathrm{CDCl}_{3}, 75.5 \mathrm{MHz}\right): \delta=40.52$ (q, $\mathrm{NMe}_{2}$ ), 47.45 (d, $\left.\mathrm{Ar}_{2} \mathrm{CH}\right), 80.03$ (t, $\mathrm{CH}_{2} \mathrm{NO}_{2}$ ), 112.79 (d), 127.58 (s), 128.24 (d), 149.70 (s); MS (EI, $70 \mathrm{eV}): m / z(\%)=313\left(\mathrm{M}^{+}, 34\right), 266$ (19), 254 (25), 253 (100), 237 (11); IR (KBr): $\widetilde{v}=2919,2806,1613,1551,1522,1357,1065,946,820 \mathrm{~cm}^{-1}$. Anal. Calcd for $\mathrm{C}_{18} \mathrm{H}_{23} \mathrm{~N}_{3} \mathrm{O}_{2}$ (313.39): C 68.98, H 7.40, N 13.41. Found C 69.21, H 7.63, N 13.38.<smiles>CNc1ccc(C(C[N+](=O)[O-])c2ccc(NC)cc2)cc1</smiles> 


\section{1,1-Bis(lilolidin-8-yl)-2-nitroethane (4a)}

$260 \mu \mathrm{L}(4.81 \mathrm{mmol})$ nitromethane (1a-H)

$1.00 \mathrm{~mL}(0.50 \mathrm{mmol}) 0.5 \mathrm{M} \mathrm{KOH}$

$208 \mathrm{mg}(0.50 \mathrm{mmol}) \mathbf{2 c}$

$0.50 \mathrm{~mL}$ acetonitrile

After $24 \mathrm{~h}$, a precipitate had formed that was collected by filtration and dried in vacuo to yield $4 \mathbf{4 a}$ (170 mg, $87 \%)$ as a pale green solid. Mp. $189-191{ }^{\circ} \mathrm{C} .{ }^{1} \mathrm{H}$ NMR $\left(\mathrm{CDCl}_{3}, 300 \mathrm{MHz}\right): \delta=2.01-$ 2.09 (m, 4 H), 2.63 (t, $J=6.5 \mathrm{~Hz}, 4 \mathrm{H}), 2.84$ (t, $J=7.9 \mathrm{~Hz}, 4 \mathrm{H}), 2.94$ (t, $J=5.5 \mathrm{~Hz}, 4 \mathrm{H}), 3.22$ (t, $J=7.7 \mathrm{~Hz}, 4 \mathrm{H}), 4.60$ (t, $\left.J=8.2 \mathrm{~Hz}, 1 \mathrm{H}, \mathrm{Ar}_{2} \mathrm{CH}\right), 4.84\left(\mathrm{~d}, J=8.2 \mathrm{~Hz}, 2 \mathrm{H}, \mathrm{CH}_{2} \mathrm{NO}_{2}\right), 6.67,6.78$ $(2 \mathrm{~s}, 2 \times 2 \mathrm{H}, \mathrm{ArH}) ;{ }^{13} \mathrm{C} \mathrm{NMR}\left(\mathrm{CDCl}_{3}, 75.5 \mathrm{MHz}\right): \delta=23.14(\mathrm{t}), 23.91(\mathrm{t}), 28.72(\mathrm{t}), 47.31(\mathrm{t})$, 48.85 (d, $\left.\mathrm{Ar}_{2} \mathrm{CH}\right), 55.19$ (t), 80.26 (t, $\mathrm{CH}_{2} \mathrm{NO}_{2}$ ), 119.39 (s), 121.18 (d), 125.44 (d), 129.24 (s), 130.25 (s), 149.21 (s); MS (EI, $70 \mathrm{eV}): m / z(\%)=389\left(\mathrm{M}^{+}, 33\right), 330$ (27), 329 (100); IR (KBr): $\widetilde{v}$ $=2944,2808,1613,1551,1494,1467,1338,1276 \mathrm{~cm}^{-1}$.

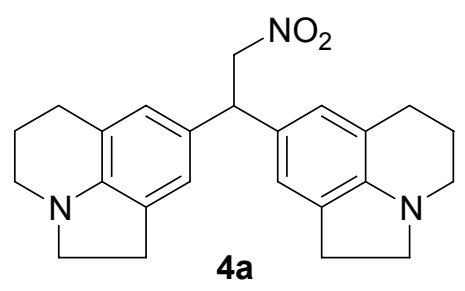

\section{1,1-Bis(4-dimethylaminophenyl)-2-nitropropane (3b)}

$350 \mu \mathrm{l}(4.90 \mathrm{mmol})$ nitroethane $(\mathbf{1 b}-\mathbf{H})$

$1.00 \mathrm{~mL}(0.50 \mathrm{mmol}) 0.5 \mathrm{M} \mathrm{KOH}$

$170 \mathrm{mg}(0.50 \mathrm{mmol}) \mathbf{2 a}$

$0.50 \mathrm{~mL}$ acetonitrile

After 20 min, a blue oil had formed which was separated from the reaction mixture. The crude product solidified upon addition of n-pentane $(30 \mathrm{~mL})$ to yield $\mathbf{3 b}(130 \mathrm{mg}, 80 \%)$ as a pale green solid. Range of melting temperatures: $128-139{ }^{\circ} \mathrm{C} .{ }^{1} \mathrm{H}$ NMR $\left(\mathrm{CDCl}_{3}, 300 \mathrm{MHz}\right): \delta=1.49(\mathrm{~d}, J=$ $\left.6.5 \mathrm{~Hz}, 3 \mathrm{H}, \mathrm{CH}_{3}\right), 2.87,2.90\left(2 \mathrm{~s}, 2 \times 6 \mathrm{H}, \mathrm{NMe}_{2}\right), 4.21\left(\mathrm{~d}, J=11.3 \mathrm{~Hz}, 1 \mathrm{H}, \mathrm{Ar}_{2} \mathrm{CH}\right), 5.23-5.33$ $\left(\mathrm{m}, 1 \mathrm{H}, \mathrm{CHNO}_{2}\right), 6.61$ (d, $\left.J=8.8 \mathrm{~Hz}, 2 \mathrm{H}, \mathrm{ArH}\right), 6.65$ (d, $\left.J=8.7 \mathrm{~Hz}, 2 \mathrm{H}, \mathrm{ArH}\right), 7.10$ (d, $J=8.8$ $\mathrm{Hz}, 2 \mathrm{H}, \mathrm{ArH}), 7.14$ (d, $J=8.7 \mathrm{~Hz}, 2 \mathrm{H}, \mathrm{ArH}) ;{ }^{13} \mathrm{C} \mathrm{NMR}\left(\mathrm{CDCl}_{3}, 75.5 \mathrm{MHz}\right): \delta=19.29\left(\mathrm{q}, \mathrm{CH}_{3}\right)$, 40.52 (q, $\mathrm{NMe}_{2}$ ), 54.69 (d, $\mathrm{Ar}_{2} \mathrm{CH}$ ), 87.39 (d, $\mathrm{CHNO}_{2}$ ), 112.78, 112.90 (2 d), 127.80 (s), 127.88 (d), 128.35 (s), 128.68 (d), 149.57, $149.60(2 \mathrm{~s}) ; \mathrm{MS}(\mathrm{EI}, 70 \mathrm{eV}): m / z(\%)=327\left(\mathrm{M}^{+}, 10\right), 254$ (20), 253 (100), 239 (10), 237 (17); IR (KBr): $\widetilde{v}=2986,2889,2803,1613,1549,1521,1446$, 
1356, 1166, 948, $808 \mathrm{~cm}^{-1}$. Anal. Calcd for $\mathrm{C}_{19} \mathrm{H}_{25} \mathrm{~N}_{3} \mathrm{O}_{2}$ (327.42): C 69.70, H 7.70, N 12.83. Found: C 69.51, H 7.57, N 12.45.<smiles>CC(C(c1ccc(N(C)C)cc1)c1ccc(N(C)C)cc1)[N+](=O)[O-]</smiles>

\section{1,1-Bis(4-dimethylaminophenyl)-2-methyl-2-nitropropane (3c)}

$440 \mu \mathrm{L}(5.00 \mathrm{mmol}) 2$-nitropropane (1c-H)

$1.00 \mathrm{~mL}(0.50 \mathrm{mmol}) 0.5 \mathrm{M} \mathrm{KOH}$

$170 \mathrm{mg}(0.50 \mathrm{mmol}) \mathbf{2 a}$

$0.50 \mathrm{~mL}$ acetonitrile

After $20 \mathrm{~min}$, a blue oil had formed which was separated from the reaction mixture. The crude product solidified upon addition of diethyl ether $(30 \mathrm{~mL})$. Further crystallizations yielded $3 \mathbf{c}(120$ $\mathrm{mg}, 70 \%)$ as pale green crystals. Mp. $140-142{ }^{\circ} \mathrm{C} .{ }^{1} \mathrm{H}$ NMR $\left(\mathrm{CDCl}_{3}, 300 \mathrm{MHz}\right): \delta=1.65(\mathrm{~s}, 6 \mathrm{H}$, $\left.\mathrm{CH}_{3}\right), 2.90\left(\mathrm{~s}, 12 \mathrm{H}, \mathrm{NMe}_{2}\right), 4.57\left(\mathrm{~s}, 1 \mathrm{H}, \mathrm{Ar}_{2} \mathrm{CH}\right), 6.63,7.20\left(2 \mathrm{~d}, \mathrm{AA}^{\prime} \mathrm{BB}^{\prime}\right.$ system with $J_{\mathrm{AB}}=8.8$ $\mathrm{Hz}, 2 \times 4 \mathrm{H}, \mathrm{ArH}) ;{ }^{13} \mathrm{C} \mathrm{NMR}\left(\mathrm{CDCl}_{3}, 75.5 \mathrm{MHz}\right): \delta=25.03\left(\mathrm{q}, \mathrm{CH}_{3}\right), 40.45\left(\mathrm{q}, \mathrm{NMe}_{2}\right), 59.01(\mathrm{~d}$, $\mathrm{Ar}_{2} \mathrm{CH}$ ), 92.91 (s, $\mathrm{CNO}_{2}$ ), 112.38 (d), 127.12 (s), 130.13 (d), 149.48 (s); MS (EI, $\left.70 \mathrm{eV}\right): \mathrm{m} / z$ (\%) $=341\left(\mathrm{M}^{+}, 4\right), 254$ (21), 253 (100), 237 (11), 83 (14); IR (KBr): $\widetilde{v}=2986,2890,2806,1614$, $1523,1348,1167,948,802 \mathrm{~cm}^{-1}$.<smiles>CNc1ccc(C(c2ccc(N(C)C)cc2)C(C)(C)[N+](=O)[O-])cc1</smiles>

\section{2,2-Bis(4-dimethylaminophenyl)-1-nitro-1-phenylethane (3d)}

$68 \mathrm{mg}(0.50 \mathrm{mmol})$ phenylnitromethane $(\mathbf{1 d - H})$

$1.00 \mathrm{~mL}(0.50 \mathrm{mmol}) 0.5 \mathrm{M} \mathrm{KOH}$

$170 \mathrm{mg}(0.50 \mathrm{mmol}) \mathbf{2 a}$

$0.50 \mathrm{~mL}$ acetonitrile

After $24 \mathrm{~h}$, a precipitate had formed which was collected by filtration to yield 3d (180 mg, 93 \%) as a pale blue solid. Mp. $135-137{ }^{\circ} \mathrm{C} .{ }^{1} \mathrm{H}$ NMR $\left(\mathrm{CDCl}_{3}, 300 \mathrm{MHz}\right): \delta=2.79,2.89(2 \mathrm{~s}, 2 \times 6 \mathrm{H}$, $\left.\mathrm{NMe}_{2}\right), 4.89\left(\mathrm{~d}, J=12.2 \mathrm{~Hz}, 1 \mathrm{H}, \mathrm{Ar}_{2} \mathrm{CH}\right), 6.17\left(\mathrm{~d}, J=12.2 \mathrm{~Hz}, 1 \mathrm{H}, \mathrm{CHNO}_{2}\right), 6.46(\mathrm{~d}, J=8.8 \mathrm{~Hz}$, 
$2 \mathrm{H}, \operatorname{ArH}), 6.67$ (d, $J=8.7 \mathrm{~Hz}, 2 \mathrm{H}, \mathrm{ArH}), 6.90$ (d, $J=8.8 \mathrm{~Hz}, 2 \mathrm{H}, \mathrm{ArH}), 7.25-7.28$ (m, $5 \mathrm{H}$, $\mathrm{ArH}), 7.48-7.51$ (m, $2 \mathrm{H}, \mathrm{ArH}) ;{ }^{13} \mathrm{C} \mathrm{NMR}\left(\mathrm{CDCl}_{3}, 75.5 \mathrm{MHz}\right): \delta=40.48,40.63\left(2 \mathrm{q}, \mathrm{NMe}_{2}\right)$, $53.26\left(\mathrm{~d}, \mathrm{Ar}_{2} \mathrm{CH}\right), 95.41$ (d, $\left.\mathrm{CHNO}_{2}\right), 112.66,113.02$ (2 d), 127.29 (s), 128.05 (d), 128.42 (d), 128.62 (s), 128.65 (d), 128.82 (d), 129.46 (d), 133.68 (s), 148.94, 149.49 (2 s); MS (EI, 70 eV): $m / z(\%)=389\left(\mathrm{M}^{+}, 2\right), 342(10), 254$ (93), 253 (100), 237 (12), 210 (19), 134 (11); IR (KBr): $\widetilde{v}=$ $2888,2805,1613,1595,1553,1521,1378,1167,937,719 \mathrm{~cm}^{-1}$.<smiles>CN(C)c1ccc(C(c2ccc(N(C)C)cc2)C(c2ccccc2)[N+](=O)[O-])cc1</smiles>

\section{2,2-Bis(4-dimethylaminophenyl)-1-(4-methylphenyl)-1-nitroethane (3e)}

$75 \mathrm{mg}(0.50 \mathrm{mmol})$ 4-methylphenylnitromethane (1e-H)

$1.00 \mathrm{~mL}(0.50 \mathrm{mmol}) 0.5 \mathrm{M} \mathrm{KOH}$

$170 \mathrm{mg}(0.50 \mathrm{mmol}) \mathbf{2 a}$

$0.50 \mathrm{~mL}$ acetonitrile

After $12 \mathrm{~h}$, a precipitate had formed that was collected by filtration to yield $\mathbf{3 e}$ (180 $\mathrm{mg}, 90 \%)$ as a pale green solid. Mp. $185-187{ }^{\circ} \mathrm{C} .{ }^{1} \mathrm{H}$ NMR $\left(\mathrm{CDCl}_{3}, 300 \mathrm{MHz}\right): \delta=2.27\left(\mathrm{~s}, 3 \mathrm{H}, \mathrm{CH}_{3}\right), 2.79,2.88$ $\left(2 \mathrm{~s}, 2 \times 6 \mathrm{H}, \mathrm{NMe}_{2}\right), 4.88\left(\mathrm{~d}, J=12.2 \mathrm{~Hz}, 1 \mathrm{H}, \mathrm{Ar}_{2} \mathrm{CH}\right), 6.15\left(\mathrm{~d}, J=12.2 \mathrm{~Hz}, 1 \mathrm{H}, \mathrm{CHNO}_{2}\right), 6.46$ $(\mathrm{d}, J=8.7 \mathrm{~Hz}, 2 \mathrm{H}, \mathrm{ArH}), 6.65(\mathrm{~d}, J=8.7 \mathrm{~Hz}, 2 \mathrm{H}, \mathrm{ArH}), 6.92(\mathrm{~d}, J=8.7 \mathrm{~Hz}, 2 \mathrm{H}, \mathrm{ArH}), 7.07$ (d, $J$ $=8.0 \mathrm{~Hz}, 2 \mathrm{H}, \mathrm{ArH}), 7.25(\mathrm{~d}, J=8.7 \mathrm{~Hz}, 2 \mathrm{H}, \operatorname{ArH}), 7.39(\mathrm{~d}, J=8.1 \mathrm{~Hz}, 2 \mathrm{H}, \operatorname{ArH}) ;{ }^{13} \mathrm{C} \mathrm{NMR}$ $\left(\mathrm{CDCl}_{3}, 75.5 \mathrm{MHz}\right): \delta=21.16\left(\mathrm{q}, \mathrm{CH}_{3}\right), 40.39,40.52\left(2 \mathrm{q}, \mathrm{NMe}_{2}\right), 53.03\left(\mathrm{~d}, \mathrm{Ar}_{2} \mathrm{CH}\right), 95.30(\mathrm{~d}$, $\mathrm{CHNO}_{2}$ ), 112.56, 112.89 (2 d), 127.34 (s), 128.05, 128.33 (2 d), 128.57 (s), 128.78 (d), 129.35 (d), 130.81 (s), 139.37 (s), 149.02, 149.61 (2 s); MS (EI, $70 \mathrm{eV}): m / z(\%)=403\left(\mathrm{M}^{+}, 2\right), 356(15), 254$ (28), 253 (100), 237 (13); IR (KBr): $\widetilde{v}=2889,2803,1614,1552,1521,1356,1165,948,807$ $\mathrm{cm}^{-1}$.<smiles>Cc1ccc(C(c2ccc(N(C)C)cc2)C(c2ccc(N(C)C)cc2)[N+](=O)[O-])cc1</smiles> 


\section{2,2-Bis(4-dimethylaminophenyl)-1-nitro-1-(4-nitrophenyl)ethane (3f)}

$91 \mathrm{mg}$ (0.50 mmol) 4-nitrophenylnitromethane (1f-H)

$1.00 \mathrm{~mL}(0.50 \mathrm{mmol}) 0.5 \mathrm{M} \mathrm{KOH}$

$170 \mathrm{mg}(0.50 \mathrm{mmol}) \mathbf{2 a}$

$1.50 \mathrm{~mL}$ acetonitrile

After $12 \mathrm{~h}$, a precipitate had formed that was collected by filtration to yield $\mathbf{3 f}$ ( $200 \mathrm{mg}, 92 \%)$ as a pale green solid. Mp. $143-145{ }^{\circ} \mathrm{C} .{ }^{1} \mathrm{H}$ NMR $\left(\mathrm{CDCl}_{3}, 300 \mathrm{MHz}\right): \delta=2.81,2.90(2 \mathrm{~s}, 2 \times 6 \mathrm{H}$, $\left.\mathrm{NMe}_{2}\right), 4.84\left(\mathrm{~d}, J=12.2 \mathrm{~Hz}, 1 \mathrm{H}, \mathrm{Ar}_{2} \mathrm{CH}\right), 6.26\left(\mathrm{~d}, J=12.2 \mathrm{~Hz}, 1 \mathrm{H}, \mathrm{CHNO}_{2}\right), 6.44(\mathrm{~d}, J=8.8 \mathrm{~Hz}$, $2 \mathrm{H}, \mathrm{ArH}), 6.67(\mathrm{~d}, J=8.8 \mathrm{~Hz}, 2 \mathrm{H}, \mathrm{ArH}), 6.88(\mathrm{~d}, J=8.6 \mathrm{~Hz}, 2 \mathrm{H}, \mathrm{ArH}), 7.25(\mathrm{~d}, J=8.1 \mathrm{~Hz}, 2 \mathrm{H}$, $\mathrm{ArH}), 7.66(\mathrm{~d}, J=8.6 \mathrm{~Hz}, 2 \mathrm{H}), \mathrm{ArH}, 8.12(\mathrm{~d}, J=8.6 \mathrm{~Hz}, 2 \mathrm{H}, \mathrm{ArH}) ;{ }^{13} \mathrm{C} \mathrm{NMR}\left(\mathrm{CDCl}_{3}, 75.5\right.$ MHz): $\delta=40.25,40.44\left(2 \mathrm{q}, \mathrm{NMe}_{2}\right), 53.91$ (d, $\left.\mathrm{Ar}_{2} \mathrm{CH}\right), 94.37\left(\mathrm{~d}, \mathrm{CHNO}_{2}\right), 112.56,112.86,123.78$ (3 d), 125.75, 127.29 (2 s), 127.89, 128.69, 129.45 (3 d), 140.32, 148.37, 149.32, 149.81 (4 s); MS (EI, $70 \mathrm{eV}): m / z(\%)=434\left(\mathrm{M}^{+},<1\right), 357$ (13), 254 (90), 253 (100), 237 (20), 210 (22), 134 (18); IR (KBr): $\widetilde{v}=2889,2804,1612,1557,1523,1348,1166,948,806 \mathrm{~cm}^{-1}$.<smiles>CN(C)c1ccc(C(c2ccc(N(C)C)cc2)C(c2ccc([N+](=O)[O-])cc2)[N+](=O)[O-])cc1</smiles>

\section{2,2-Bis(4-dimethylaminophenyl)-1-nitro-1-(3-nitrophenyl)ethane (3g)}

$91 \mathrm{mg}(0.50 \mathrm{mmol}) 3$-nitrophenylnitromethane (1g-H)

$1.00 \mathrm{~mL}(0.50 \mathrm{mmol}) 0.5 \mathrm{M} \mathrm{KOH}$

$170 \mathrm{mg}(0.50 \mathrm{mmol}) \mathbf{2 a}$

$1.50 \mathrm{~mL}$ acetonitrile

After $48 \mathrm{~h}$, a precipitate had formed that was collected by filtration to yield $\mathbf{3 g}$ ( $170 \mathrm{mg}, 78 \%$ ) as a yellow-green solid. Mp. 142-144 ${ }^{\circ} \mathrm{C} .{ }^{1} \mathrm{H}$ NMR $\left(\mathrm{CDCl}_{3}, 300 \mathrm{MHz}\right): \delta=2.80,2.90(2 \mathrm{~s}, 2 \times 6 \mathrm{H}$, $\mathrm{NMe}_{2}$ ), 4.87 (d, $\left.J=12.2 \mathrm{~Hz}, 1 \mathrm{H}, \mathrm{Ar}_{2} \mathrm{CH}\right), 6.27$ (d, $J=12.2 \mathrm{~Hz}, 1 \mathrm{H}, \mathrm{CHNO}_{2}$ ), 6.45, 6.67, 6.91,

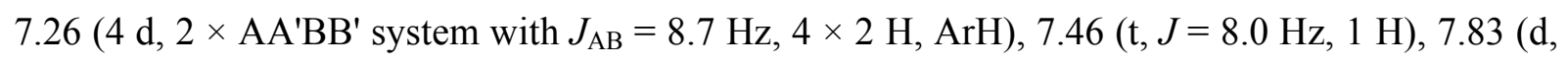
$J=7.7 \mathrm{~Hz}, 1 \mathrm{H}), 8.12-8.16(\mathrm{~m}, 1 \mathrm{H}), 8.38-8.39(\mathrm{~m}, 1 \mathrm{H}) ;{ }^{13} \mathrm{C} \mathrm{NMR}\left(\mathrm{CDCl}_{3}, 75.5 \mathrm{MHz}\right): \delta=$ 40.27, 40.44 (2 q, $\mathrm{NMe}_{2}$ ), 53.67 (d, $\left.\mathrm{Ar}_{2} \mathrm{CH}\right), 94.28$ (d, $\left.\mathrm{CHNO}_{2}\right), 112.65,112.86,123.61,124.44$ (4 d), 125.84, $127.24(2 \mathrm{~s}), 127.93,128.69,129.73,134.41$ (4 d), 135.62, 148.19, 149.32, 149.81 (4 
s); MS (EI, $70 \mathrm{eV}): m / z(\%)=434\left(\mathrm{M}^{+},<1\right), 387$ (9), 254 (79), 253 (100), 237 (20), 210 (17), IR $(\mathrm{KBr}): \widetilde{v}=3083,2888,2803,1613,1557,1527,1351,1166,948,806 \mathrm{~cm}^{-1}$.

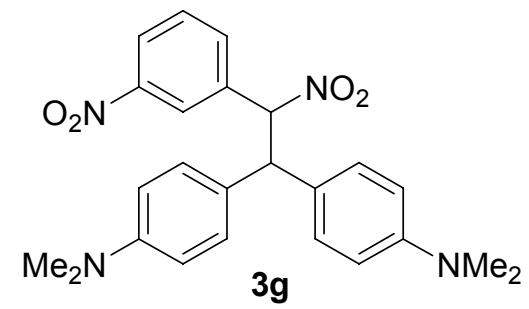




\subsection{Reactions of the nitroethane anion $\left(1 \mathrm{~b}-\mathrm{NBu}_{4}{ }^{+}\right)$with quinone methides $2 \mathrm{f}-\mathrm{h}$}

At room temperature (ca. $\left.20{ }^{\circ} \mathrm{C}\right)$, a solution of $1 \mathbf{b}-\mathrm{NBu}_{4}{ }^{+}(475 \mathrm{mg}, 1.50 \mathrm{mmol})$ in DMSO was mixed with a solution of quinone methide $\mathbf{2} \mathbf{f}-\mathbf{h}(1.00 \mathrm{mmol})$ in dichloromethane. After $30 \mathrm{~min}$, water $(30 \mathrm{~mL})$ was added, and the solution was neutralized with aq. $\mathrm{HCl}(2 \mathrm{M})$. The aqueous layer was extracted with $\mathrm{Et}_{2} \mathrm{O}(4 \times 50 \mathrm{~mL})$, the combined organic layers were washed with water $(4 \times$ $50 \mathrm{~mL}$ ) and dried over $\mathrm{MgSO}_{4}$. After evaporation of the solvent, the products $\mathbf{5 f - h}$ were purified as described in for experiment.

Reaction time and purification as described in each experiment. All reactions were performed at room temperature (ca. $\left.20^{\circ} \mathrm{C}\right)$. The conditions of the reactions were not optimized for high yields.

2,6-Di-tert-butyl-4-(2-nitro-1-(4-methylphenyl)propyl)phenol (5f). The reaction of quinone methide $2 \mathbf{f}\left(308 \mathrm{mg}, 1.00 \mathrm{mmol}\right.$, dissolved in $10 \mathrm{~mL} \mathrm{CH}_{2} \mathrm{Cl}_{2}$ ) and $\mathbf{1 b}-\mathrm{NBu}_{4}{ }^{+}$(dissolved in $5 \mathrm{~mL}$ DMSO) gave rise to the formation of an oily crude product which was dissolved in $n$-pentane and recrystallized at $-20{ }^{\circ} \mathrm{C}$ to yield $\mathbf{5 f}(80 \mathrm{mg}, 19 \%)$ as pale orange crystals. ${ }^{1} \mathrm{H}$ NMR $(300 \mathrm{MHz}$, $\left.\mathrm{CDCl}_{3}\right)$ : Mixture of diastereomers: $\delta=1.38,1.40\left(2 \mathrm{~s}, 18 \mathrm{H}, \mathrm{C}\left(\mathrm{CH}_{3}\right)_{3}\right), 1.48(\mathrm{~d}, J=6.5 \mathrm{~Hz}, 3 \mathrm{H}$, $\left.\mathrm{CH}_{3}\right), 2.27,2.30\left(2 \mathrm{~s}, 3 \mathrm{H}, \mathrm{ArCH}_{3}\right), 4.24,4.27\left(2 \mathrm{~d}, J=11.4\right.$ and $\left.11.3 \mathrm{~Hz}, 1 \mathrm{H}, \mathrm{Ar}_{2} \mathrm{CH}\right), 5.05,5.11$ $(2 \mathrm{~s}, 1 \mathrm{H}, \mathrm{OH}), 5.25-5.35\left(\mathrm{~m}, 1 \mathrm{H}, \mathrm{CHNO}_{2}\right), 7.03-7.23(\mathrm{~m}, 6 \mathrm{H}) ;{ }^{13} \mathrm{C} \mathrm{NMR}\left(75.5 \mathrm{MHz}, \mathrm{CDCl}_{3}\right): \delta$ $=19.32\left(\mathrm{q}, \mathrm{CH}_{3}\right), 20.97$ (q, $\left.\mathrm{ArCH}_{3}\right), 30.24\left(\mathrm{q}, \mathrm{C}\left(\mathrm{CH}_{3}\right)_{3}\right), 34.34\left(\mathrm{~s}, \mathrm{C}\left(\mathrm{CH}_{3}\right)_{3}\right), 56.14\left(\mathrm{~d}, \mathrm{Ar}_{2} \mathrm{CH}\right)$, 87.42 (d, $\mathrm{CHNO}_{2}$ ), 123.84, 124.45 (2 d), 127.18, 127.91 (2 d), 129.67, 129.44 (2 d), 129.93, 130.41 (2 s), 135.82, 136.34 (2 s), 136.76 (s), 137.42 (s), 152.95 (s). Anal. Calcd for $\mathrm{C}_{24} \mathrm{H}_{33} \mathrm{NO}_{3}$ (383.53): C 75.16, H 8.67, N 3.65. Found C 75.06, H 8.60, N 3.60.<smiles>Cc1ccc(C(c2cc(C(C)(C)C)c(O)c(C(C)(C)C)c2)C(C)[N+](=O)[O-])cc1</smiles>

2,6-Di-tert-butyl-4-(1-(4-methoxyphenyl)-2-nitropropyl)phenol (5g). The reaction of quinone methide $\mathbf{2 g}$ (324 mg, $1.00 \mathrm{mmol}$, dissolved in $15 \mathrm{~mL} \mathrm{CH}_{2} \mathrm{Cl}_{2}$ ) and $\mathbf{1 b}-\mathrm{NBu}_{4}{ }^{+}$(dissolved in $30 \mathrm{~mL}$ DMSO) yielded a crude product which was recrystallized from methanol to give $\mathbf{5 g}(220 \mathrm{mg}, 55$ \%) as colorless crystals. M.p. $163-164{ }^{\circ} \mathrm{C} ;{ }^{1} \mathrm{H}$ NMR $\left(300 \mathrm{MHz}, \mathrm{CDCl}_{3}\right)$ : Mixture of diastereomeres (9:1) $\delta=1.38,1.40\left(2 \mathrm{~s}, 18 \mathrm{H}, \mathrm{C}\left(\mathrm{CH}_{3}\right)_{3}\right), 1.48\left(\mathrm{~d}, J=6.5 \mathrm{~Hz}, 3 \mathrm{H}, \mathrm{CH}_{3}\right), 3.73,3.76$ 
$(2 \mathrm{~s}, 3 \mathrm{H}, \mathrm{OMe}), 4.24\left(\mathrm{~d}, J=11.4 \mathrm{~Hz}, 1 \mathrm{H}, \mathrm{Ar}_{2} \mathrm{CH}\right), 5.06,5.12(2 \mathrm{~s}, 1 \mathrm{H}, \mathrm{OH}), 5.33-5.32(\mathrm{~m}, 1 \mathrm{H}$, $\left.\mathrm{CHNO}_{2}\right), 6.78-6.86(\mathrm{~m}, 2 \mathrm{H}), 7.02,7.06(2 \mathrm{~s}, 2 \mathrm{H}), 7.16-7.25(\mathrm{~m}, 2 \mathrm{H}) ;{ }^{13} \mathrm{C}$ NMR $(75.5 \mathrm{MHz}$, $\left.\mathrm{CDCl}_{3}\right): \delta=19.30\left(\mathrm{q}, \mathrm{CH}_{3}\right), 30.24\left(\mathrm{q}, \mathrm{C}\left(\mathrm{CH}_{3}\right)_{3}\right), 34.34\left(\mathrm{~s}, \mathrm{C}\left(\mathrm{CH}_{3}\right)_{3}\right), 55.13,55.53,55.69\left(\mathrm{Ar}_{2} \mathrm{CH}\right.$ and $\mathrm{OMe}), 87.16,87.56$ ( $\left.2 \mathrm{~d}, \mathrm{CHNO}_{2}\right), 114.13,114.36$ (2 d), 123.81, 124.43 (2 d), 128.38, 129.08 (2 d), 130.02, 130.50 (2 s), 132.19, 132.51 (2 s), 135.84, 136.34 (2 s), 152.91 (s), 158.61 (s); MS (EI, $70 \mathrm{eV}): m / z(\%)=399\left(\mathrm{M}^{+}, 8\right), 352(15), 326(22), 325(100)$. Anal. Calcd for $\mathrm{C}_{24} \mathrm{H}_{33} \mathrm{NO}_{4}$ (399.52): C 72.15, H 8.33, N 3.51. Found C 72.29, H 8.43, N 3.37.<smiles>COc1ccc(C(c2cc(C(C)(C)C)c(O)c(C(C)(C)C)c2)C(C)[N+](=O)[O-])cc1</smiles>

$5 \mathrm{~g}$

2,6-Di-tert-butyl-4-[1-(4-dimethylaminophenyl)-2-nitropropyl]-phenol (5h). The reaction of quinone methide $\mathbf{2 h}$ (338 mg, $1.00 \mathrm{mmol}$, dissolved in $10 \mathrm{~mL} \mathrm{CH}_{2} \mathrm{Cl}_{2}$ ) and $\mathbf{1 b}-\mathrm{NBu}_{4}{ }^{+}$(dissolved in $20 \mathrm{~mL}$ DMSO) yielded a crude product which was recrystallized from 2-propanol to yield $\mathbf{5 h}$ (179 mg, $43 \%)$ as a pale red solid. ${ }^{1} \mathrm{H}$ NMR $\left(300 \mathrm{MHz}, \mathrm{CDCl}_{3}\right)$ : Mixture of diastereomeres (6:4): $\delta=$ 1.38, $1.40\left(2 \mathrm{~s}, 18 \mathrm{H}, \mathrm{C}\left(\mathrm{CH}_{3}\right)_{3}\right), 1.46\left(\mathrm{~d}, J=6.5 \mathrm{~Hz}, 3 \mathrm{H}, \mathrm{CH}_{3}\right), 2.87,2.90\left(2 \mathrm{~s}, 6 \mathrm{H}, \mathrm{NMe}_{2}\right), 4.18$, $4.20\left(2 \mathrm{~d}, J=11.3 \mathrm{~Hz}, 1 \mathrm{H}, \mathrm{Ar}_{2} \mathrm{CH}\right), 5.02,5.08,(2 \mathrm{~s}, 1 \mathrm{H}, \mathrm{OH}), 5.22-5.34\left(\mathrm{~m}, 1 \mathrm{H}, \mathrm{CHNO}_{2}\right)$, 6.60-6.68 (m, $2 \mathrm{H}), 7.03,7.07(2 \mathrm{~s}, 2 \mathrm{H}), 7.10-7.19(\mathrm{~m}, 2 \mathrm{H}) ;{ }^{13} \mathrm{C} \mathrm{NMR}\left(75.5 \mathrm{MHz}, \mathrm{CDCl}_{3}\right): \delta=$ 19.30, 19.33 (2 q, $\left.\mathrm{CH}_{3}\right), 30.25$ (q, $\left.\mathrm{C}\left(\mathrm{CH}_{3}\right)_{3}\right), 34.26,34.20\left(2 \mathrm{~s}, \mathrm{C}\left(\mathrm{CH}_{3}\right)_{3}\right), 40.42$ (q, $\left.\mathrm{NMe}_{2}\right), 55.59$, $55.73\left(2 \mathrm{~d}, \mathrm{Ar}_{2} \mathrm{CH}\right), 87.37,87.75\left(2 \mathrm{~d}, \mathrm{CHNO}_{2}\right), 112.70,112.82$ (2 d), 123.79, 124.38 (2 d), 127.74, 128.14 (2 s), 127.99, 128.69 (2 d), 130.52, 130.93 (2 s), 135.68, 136.20 (2 s), 149.59 (s), $152.64,152.74(2 \mathrm{~s})$.<smiles>CC(c1cc(C(C)(C)C)c(O)c(C(C)(C)C)c1)C(c1ccc(N(C)C)cc1)[N+](=O)[O-]</smiles>

$5 \mathrm{~h}$ 


\section{Kinetic Investigations}

- As the reactions of the colored electrophiles $2 \mathbf{a}-\mathbf{i}$ (benzhydrylium ions and the quinone methides) with the nitroalkyl anions gave rise to colorless products, the decay of the electrophile absorptions was followed by UV-Vis-spectroscopy.

- The rates of slow reactions $\left(\tau_{1 / 2}>10 \mathrm{~s}\right)$ were determined by using a J\&M TIDAS diode array spectrophotometer which was controlled by Labcontrol Spectacle software and connected to a Hellma 661.502-QX quartz Suprasil immersion probe (5 mm light path) via fiber optic cables and standard SMA connectors. The temperature of the solutions during all kinetic studies was kept constant $\left(20.0 \pm 0.2{ }^{\circ} \mathrm{C}\right)$ by using a circulating bath thermostat and was monitored with a thermo-couple probe that was inserted into the reaction mixture. $^{47,48}$

- For the evaluation of fast $\left(\tau_{1 / 2}<10 \mathrm{~s}\right)$ stopped-flow kinetics (stopped-flow spectrophotometer system Hi-Tech SF-61DX2 controlled by Hi-Tech KinetAsyst2 software), rate constants $k_{1 \psi, o b s}\left(20.0 \pm 0.2{ }^{\circ} \mathrm{C}\right)$ were obtained by fitting the single exponential $A_{t}=A_{0} \exp \left(-k_{1 \psi, \mathrm{obs}} t\right)+C$ to the observed time-dependent carbocation absorbance (averaged from at least 4 kinetic runs at each nucleophile concentration. ${ }^{47,48}$

\subsection{Kinetic investigations of nitroalkyl anions in water}

\subsubsection{Remarks}

- In the experiments with J\&M TIDAS $\left(\tau_{1 / 2}>10 \mathrm{~s}\right)$ three stock solutions have been used: A solution of the electrophile in $\mathrm{CH}_{3} \mathrm{CN}$, the $\mathrm{CH}$ acid in water (with one drop $\mathrm{CH}_{3} \mathrm{CN}$ ) and the commercially available aqueous $\mathrm{KOH}$ solution. In the experiments with the most reactive electrophile $\mathbf{2 a}$, first the nitroalkyl anion was produced from the $\mathrm{CH}$ acid and $\mathrm{KOH}$. Data acquisition was started after the addition of $\mathbf{2 a}$. In all other cases, the order was: electrophile, $\mathrm{CH}$ acid, $\mathrm{KOH}$.

- For the stopped-flow experiments $\left(\tau_{1 / 2}<10 \mathrm{~s}\right)$ two stock solutions were used: An aqueous solution (with $\mathrm{CH}_{3} \mathrm{CN}$ as a cosolvent) of the electrophile and the second flask with an aqueous solution of the nitroalkyl anions generated from the $\mathrm{CH}$ acid and the aqueous $\mathrm{KOH}$.

- In the experiments with the most reactive electrophile 2a, small quantities of benzenesulfonic acid (BSA) were added to stabilize the aqueous stock solutions. In those 
cases $\left[\mathrm{OH}^{-}\right]_{0}$ is the remaining $\mathrm{OH}^{-}$concentration after neutralization by benzenesulfonic acid (BSA).

- As shown on the following pages, the plots of $k_{1 \psi}\left(k_{1 \psi}=k_{1 \psi, \mathrm{obs}}-k_{1 \psi}, \mathrm{OH}^{-}\right)$increases linearly with the concentration of the nucleophile, and the slopes of the correlations correspond to the second-order rate-constants $\left(k_{2}\right)$.

\subsubsection{Calculation of the nitroalkyl anion concentration $\left[\mathrm{C}^{-}\right]$in aqueous solutions ${ }^{22}$}

$$
\mathrm{CH} \text { acid }+\mathrm{OH}^{-} \rightleftharpoons \mathrm{C}^{-}+\mathrm{H}_{2} \mathrm{O}
$$

1) $K_{\mathrm{B}}=\left([\mathrm{CH}\right.$ acid $\left.]\left[\mathrm{OH}^{-}\right]\right) /\left[\mathrm{C}^{-}\right]$

2) $\left[\mathrm{OH}^{-}\right]_{0}=\left[\mathrm{OH}^{-}\right]+\left[\mathrm{C}^{-}\right]$

$$
\left[\mathrm{C}^{-}\right]=\left[\mathrm{OH}^{-}\right]_{0}-\left[\mathrm{OH}^{-}\right]
$$

3) $[\mathrm{CH} \text { acid }]_{0}=[\mathrm{CH}$ acid $]+\left[\mathrm{C}^{-}\right]$

$$
\begin{aligned}
& {[\mathrm{CH} \text { acid }]=[\mathrm{CH} \text { acid }]_{0}-\left[\mathrm{C}^{-}\right] \quad \text { 2.1) in 3) }} \\
& {[\mathrm{CH} \text { acid }]=[\mathrm{CH} \text { acid }]_{0}-\left[\mathrm{OH}^{-}\right]_{0}+\left[\mathrm{OH}^{-}\right] \quad \text { | 3.1) }}
\end{aligned}
$$

2.1) and 3.1) in 1)

$K_{\mathrm{B}}=\left(\left([\mathrm{CH} \text { acid }]_{0}-\left[\mathrm{OH}^{-}\right]_{0}+\left[\mathrm{OH}^{-}\right]\right)\left[\mathrm{OH}^{-}\right]\right) /\left(\left[\mathrm{OH}^{-}\right]_{0}-\left[\mathrm{OH}^{-}\right]\right)$

$K_{\mathrm{B}}\left[\mathrm{OH}^{-}\right]_{0}-K_{\mathrm{B}}\left[\mathrm{OH}^{-}\right]=\left[\mathrm{OH}^{-}\right]^{2}+\left[\mathrm{OH}^{-}\right]\left([\mathrm{CH} \text { acid }]_{0}-\left[\mathrm{OH}^{-}\right]_{0}\right)$

$0=\left[\mathrm{OH}^{-}\right]^{2}+\left[\mathrm{OH}^{-}\right]\left([\mathrm{CH} \text { acid }]_{0}-\left[\mathrm{OH}^{-}\right]_{0}+K_{\mathrm{B}}\right)-K_{\mathrm{B}}\left[\mathrm{OH}^{-}\right]_{0}$

Solving the quadratic equation leads to one logic solution of $\left[\mathrm{OH}^{-}\right]$(The one with the "+" in the numerator).

$$
\left[\mathrm{OH}^{-}\right]=1 / 2\left(-\left([\mathrm{CH} \text { acid }]_{0}-\left[\mathrm{OH}^{-}\right]_{0}+K_{\mathrm{B}}\right)+\left(\left([\mathrm{CH} \text { acid }]_{0}-\left[\mathrm{OH}^{-}\right]_{0}+K_{\mathrm{B}}\right)^{2}+4 K_{\mathrm{B}}\left[\mathrm{OH}^{-}\right]_{0}\right)^{1 / 2}\right)
$$

$\left[\mathrm{C}^{-}\right]$can then be calculated with 2.1):

2.1) $\left[\mathrm{C}^{-}\right]=\left[\mathrm{OH}^{-}\right]_{0}-\left[\mathrm{OH}^{-}\right]$ 


\subsubsection{Individual kinetic runs in water (Tables S1-S26)}

Benzhydrylium ions $\mathbf{2}$ were used as tetrafluoroborate salts. BSA = benzenesulfonic acid.

Table S1. Potassium salt of nitromethide (1a), 2a, and benzenesulfonic acid (BSA) at $605 \mathrm{~nm}$ at $20 \mathrm{C}$ in water (J\&M).

\begin{tabular}{rccccc}
\hline \multicolumn{1}{c}{ No. } & $\begin{array}{c}{[\mathbf{2 a}]_{0} /} \\
\mathrm{mol} \mathrm{L}^{-1}\end{array}$ & $\begin{array}{c}{[\mathrm{BSA}] /} \\
\mathrm{mol} \mathrm{L}\end{array}$ & $\begin{array}{c}{\left[\mathrm{OH}^{-1}\right]_{0} /} \\
\mathrm{mol} \mathrm{L}^{-1, a}\end{array}$ & $\begin{array}{c}{[\mathbf{1 a - H}]_{0} /} \\
\mathrm{mol} \mathrm{L}^{-1}\end{array}$ & $\begin{array}{c}{\left[\mathrm{OH}^{-}\right] /} \\
\mathrm{mol} \mathrm{L}^{-1}\end{array}$ \\
\hline BUT-194-2 & $3.18 \times 10^{-5}$ & $3.55 \times 10^{-5}$ & $1.62 \times 10^{-4}$ & $2.26 \times 10^{-3}$ & $1.18 \times 10^{-5}$ \\
BUT-194-3 & $3.31 \times 10^{-5}$ & $3.70 \times 10^{-5}$ & $2.71 \times 10^{-4}$ & $3.53 \times 10^{-3}$ & $1.31 \times 10^{-5}$ \\
BUT-194-4 & $3.26 \times 10^{-5}$ & $3.64 \times 10^{-5}$ & $3.68 \times 10^{-4}$ & $4.63 \times 10^{-3}$ & $1.37 \times 10^{-5}$ \\
BUT-194-5 & $3.23 \times 10^{-5}$ & $3.61 \times 10^{-5}$ & $4.65 \times 10^{-4}$ & $5.74 \times 10^{-3}$ & $1.41 \times 10^{-5}$ \\
\hline No. & {$[\mathbf{1 a}] /$} & $k_{1 \Psi, \text { obs }} /$ & Conv. / & $k_{1 \Psi, \mathrm{OH}^{-} /}$ & $k_{1 \Psi} /$ \\
\hline BUT-194-2 & $1.50 \times 10^{-4}$ & $1.02 \times 10^{-1}$ & 80 & $1.54 \times 10^{-3}$ & $1.00 \times 10^{-1}$ \\
BUT-194-3 & $2.58 \times 10^{-4}$ & $1.63 \times 10^{-1}$ & 92 & $1.71 \times 10^{-3}$ & $1.61 \times 10^{-1}$ \\
BUT-194-4 & $3.54 \times 10^{-4}$ & $2.12 \times 10^{-1}$ & 92 & $1.80 \times 10^{-3}$ & $2.10 \times 10^{-1}$ \\
BUT-194-5 & $4.51 \times 10^{-4}$ & $2.57 \times 10^{-1}$ & 95 & $1.85 \times 10^{-3}$ & $2.55 \times 10^{-1}$ \\
\hline
\end{tabular}

${ }^{a}$ Remaining $\left[\mathrm{OH}^{-}\right]_{0}$ after neutralization by benzenesulfonic acid (BSA).

${ }^{b}$ Calculated with $k_{2, \mathrm{OH}^{-}}=131 \times 10^{2} \mathrm{~L} \mathrm{~mol}^{-1} \mathrm{~s}^{-1}$ from ref 11 .

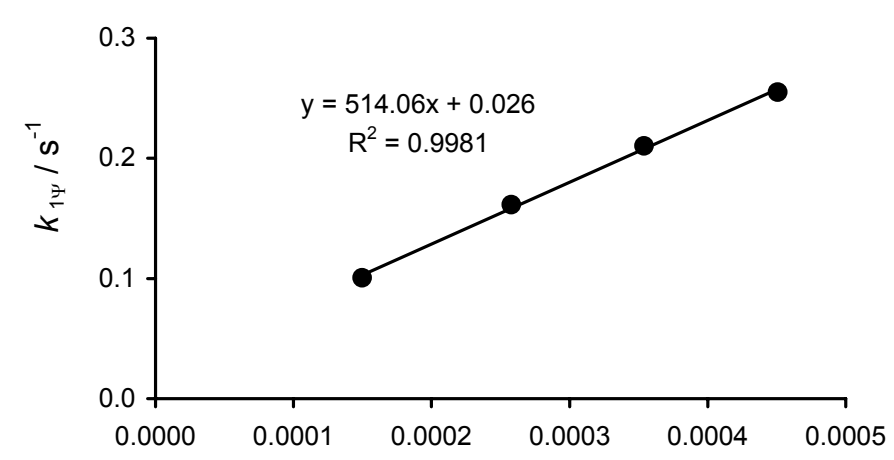

$[\mathrm{C}] / \mathrm{mol} \mathrm{L}^{-1}$

$k_{2, \mathrm{C}^{-}}=5.14 \times 10^{2} \mathrm{~L} \mathrm{~mol}^{-1} \mathrm{~s}^{-1}$ 
Table S2. Potassium salt of nitromethide (1a) and $\mathbf{2 b}$ at $620 \mathrm{~nm}$ at $20{ }^{\circ} \mathrm{C}$ in water (J\&M).

\begin{tabular}{rcccc}
\hline \multicolumn{1}{c}{ No. } & $\begin{array}{c}{[\mathbf{2 b}]_{0} /} \\
\mathrm{mol} \mathrm{L}^{-1}\end{array}$ & $\begin{array}{c}{\left[\mathrm{OH}^{-}\right]_{0} /} \\
\mathrm{mol} \mathrm{L}^{-1}\end{array}$ & $\begin{array}{c}{[\mathbf{1 a - H}]_{0} /} \\
\mathrm{mol} \mathrm{L}^{-1}\end{array}$ & $\begin{array}{c}{[\mathbf{1 a}] /} \\
\mathrm{mol} \mathrm{L}^{-1}\end{array}$ \\
\hline BUT-193-1 & $2.34 \times 10^{-5}$ & $7.76 \times 10^{-5}$ & $8.13 \times 10^{-4}$ & $6.35 \times 10^{-5}$ \\
BUT-193-2 & $2.55 \times 10^{-5}$ & $2.11 \times 10^{-4}$ & $2.21 \times 10^{-3}$ & $1.95 \times 10^{-4}$ \\
BUT-193-3 & $2.53 \times 10^{-5}$ & $4.19 \times 10^{-4}$ & $4.39 \times 10^{-3}$ & $4.02 \times 10^{-4}$ \\
BUT-193-4 & $2.31 \times 10^{-5}$ & $7.66 \times 10^{-4}$ & $8.02 \times 10^{-3}$ & $7.49 \times 10^{-4}$ \\
BUT-193-5 & $2.48 \times 10^{-5}$ & $1.23 \times 10^{-3}$ & $1.29 \times 10^{-2}$ & $1.21 \times 10^{-3}$ \\
BUT-193-6 & $2.20 \times 10^{-5}$ & $1.46 \times 10^{-3}$ & $1.53 \times 10^{-2}$ & $1.44 \times 10^{-3}$ \\
\hline No. & $k_{1 \Psi, \text { obs } /}$ & $\mathrm{Conv} . /$ & $k_{1 \Psi, \mathrm{OH}^{-} /}$ & $k_{1 \Psi} /$ \\
\hline BUT-193-1 & $8.62 \times 10^{-3}$ & 63 & $3.32 \times 10^{-4}$ & $8.29 \times 10^{-3}$ \\
BUT-193-2 & $2.15 \times 10^{-2}$ & 92 & $3.79 \times 10^{-4}$ & $2.11 \times 10^{-2}$ \\
BUT-193-3 & $4.15 \times 10^{-2}$ & 98 & $3.95 \times 10^{-4}$ & $4.11 \times 10^{-2}$ \\
BUT-193-4 & $7.89 \times 10^{-2}$ & 92 & $4.03 \times 10^{-4}$ & $7.85 \times 10^{-2}$ \\
BUT-193-5 & $1.29 \times 10^{-1}$ & 89 & $4.06 \times 10^{-4}$ & $1.29 \times 10^{-1}$ \\
BUT-193-6 & $1.52 \times 10^{-1}$ & 96 & $4.07 \times 10^{-4}$ & $1.52 \times 10^{-1}$ \\
\hline
\end{tabular}

${ }^{b}$ Calculated with $k_{2, \mathrm{OH}^{-}}=2.36 \times 10^{1} \mathrm{~L} \mathrm{~mol}^{-1} \mathrm{~s}^{-1}$ from ref 11 .

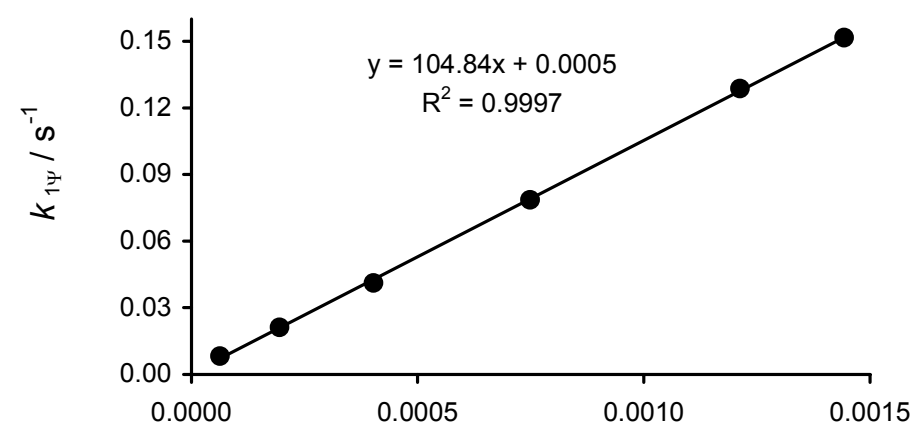

$\left[\mathrm{C}^{-}\right] / \mathrm{mol} \mathrm{L}^{-1}$

$k_{2, \mathrm{C}^{-}}=1.05 \times 10^{2} \mathrm{~L} \mathrm{~mol}^{-1} \mathrm{~s}^{-1}$ 
Table S3. Potassium salt of nitromethide (1a) and $\mathbf{2 b}$ at $620 \mathrm{~nm}$ at $20{ }^{\circ} \mathrm{C}$ in water with $\left[\mathrm{OH}^{-}\right]_{0}=$ constant $=2.54 \times 10^{-3}$ (stopped-flow).

\begin{tabular}{|c|c|c|c|c|}
\hline No. & $\begin{array}{c}{[\mathbf{2} \mathbf{b}]_{0} /} \\
\mathrm{mol} \mathrm{L}^{-1}\end{array}$ & $\begin{array}{l}{\left[\mathrm{OH}^{-}\right]_{0} /} \\
\mathrm{mol} \mathrm{L}^{-1}\end{array}$ & $\begin{array}{c}{[\mathbf{1} \mathbf{a}-\mathbf{H}]_{0} /} \\
\mathrm{mol} \mathrm{L}^{-1} \\
\end{array}$ & $\begin{array}{c}{[\mathbf{1 a}] /} \\
\mathrm{mol} \mathrm{L}^{-1}\end{array}$ \\
\hline BUT-191-7 & $9.79 \times 10^{-6}$ & $2.54 \times 10^{-3}$ & $3.66 \times 10^{-4}$ & $3.40 \times 10^{-4}$ \\
\hline BUT-191-8 & $9.79 \times 10^{-6}$ & $2.54 \times 10^{-3}$ & $5.48 \times 10^{-4}$ & $5.07 \times 10^{-4}$ \\
\hline BUT-191-9 & $9.79 \times 10^{-6}$ & $2.54 \times 10^{-3}$ & $7.31 \times 10^{-4}$ & $6.71 \times 10^{-4}$ \\
\hline BUT-191-10 & $9.79 \times 10^{-6}$ & $2.54 \times 10^{-3}$ & $9.14 \times 10^{-4}$ & $8.33 \times 10^{-4}$ \\
\hline BUT-191-11 & $9.79 \times 10^{-6}$ & $2.54 \times 10^{-3}$ & $1.37 \times 10^{-3}$ & $1.22 \times 10^{-3}$ \\
\hline BUT-191-12 & $9.79 \times 10^{-6}$ & $2.54 \times 10^{-3}$ & $1.83 \times 10^{-3}$ & $1.56 \times 10^{-3}$ \\
\hline No. & $\begin{array}{l}{\left[\mathrm{OH}^{-}\right] /} \\
\mathrm{mol} \mathrm{L}^{-1}\end{array}$ & $\begin{array}{c}k_{1 \Psi, \mathrm{obs}} / \\
\mathrm{s}^{-1} \\
\end{array}$ & $\begin{array}{c}k_{1 \Psi, \mathrm{OH}^{-}} / \\
\mathrm{s}^{-1, a} \\
\end{array}$ & $\begin{array}{c}k_{1 \Psi} / \\
\mathrm{s}^{-1} \\
\end{array}$ \\
\hline BUT-191-7 & $2.20 \times 10^{-3}$ & $8.64 \times 10^{-2}$ & $5.19 \times 10^{-2}$ & $3.44 \times 10^{-2}$ \\
\hline BUT-191-8 & $2.03 \times 10^{-3}$ & $9.73 \times 10^{-2}$ & $4.80 \times 10^{-2}$ & $4.93 \times 10^{-2}$ \\
\hline BUT-191-9 & $1.87 \times 10^{-3}$ & $1.12 \times 10^{-1}$ & $4.41 \times 10^{-2}$ & $6.77 \times 10^{-2}$ \\
\hline BUT-191-10 & $1.71 \times 10^{-3}$ & $1.25 \times 10^{-1}$ & $4.03 \times 10^{-2}$ & $8.49 \times 10^{-2}$ \\
\hline BUT-191-11 & $1.32 \times 10^{-3}$ & $1.54 \times 10^{-1}$ & $3.12 \times 10^{-2}$ & $1.22 \times 10^{-1}$ \\
\hline BUT-191-12 & $9.76 \times 10^{-4}$ & $1.83 \times 10^{-1}$ & $2.30 \times 10^{-2}$ & $1.60 \times 10^{-1}$ \\
\hline
\end{tabular}

${ }^{a}$ Calculated with $k_{2, \mathrm{OH}^{-}}=2.36 \times 10^{1} \mathrm{~L} \mathrm{~mol}^{-1} \mathrm{~s}^{-1}$ from ref 11 .

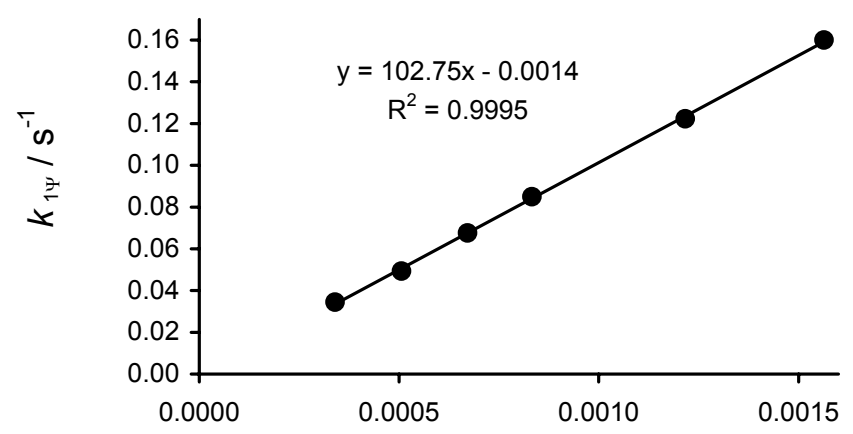

$[\mathrm{C}] / \mathrm{mol} \mathrm{L}^{-1}$

$k_{2, \mathrm{C}^{-}}=1.03 \times 10^{2} \mathrm{~L} \mathrm{~mol}^{-1} \mathrm{~s}^{-1}$ 
Table S4. Potassium salt of nitromethide (1a) and $\mathbf{2 b}$ at $620 \mathrm{~nm}$ at $20{ }^{\circ} \mathrm{C}$ in water with $\left[\mathrm{OH}^{-}\right]_{0}=$ constant $=5.07 \times 10^{-3}$ (stopped-flow).

\begin{tabular}{ccccc}
\hline \multicolumn{1}{c}{ No. } & $\begin{array}{c}{[\mathbf{2 b}]_{0} /} \\
\mathrm{mol} \mathrm{L}^{-1}\end{array}$ & $\begin{array}{c}{\left[\mathrm{OH}^{-}\right]_{0} /} \\
\mathrm{mol} \mathrm{L}^{-1}\end{array}$ & $\begin{array}{c}{[\mathbf{1 a - H}]_{0} /} \\
\mathrm{mol} \mathrm{L}^{-1}\end{array}$ & $\begin{array}{c}{[\mathbf{1 a}] /} \\
\mathrm{mol} \mathrm{L}^{-1}\end{array}$ \\
\hline BUT-191-1 & $9.79 \times 10^{-6}$ & $5.07 \times 10^{-3}$ & $3.66 \times 10^{-4}$ & $3.54 \times 10^{-4}$ \\
BUT-191-2 & $9.79 \times 10^{-6}$ & $5.07 \times 10^{-3}$ & $5.48 \times 10^{-4}$ & $5.29 \times 10^{-4}$ \\
BUT-191-3 & $9.79 \times 10^{-6}$ & $5.07 \times 10^{-3}$ & $7.31 \times 10^{-4}$ & $7.04 \times 10^{-4}$ \\
BUT-191-4 & $9.79 \times 10^{-6}$ & $5.07 \times 10^{-3}$ & $9.14 \times 10^{-4}$ & $8.79 \times 10^{-4}$ \\
BUT-191-5 & $9.79 \times 10^{-6}$ & $5.07 \times 10^{-3}$ & $1.37 \times 10^{-3}$ & $1.31 \times 10^{-3}$ \\
BUT-191-6 & $9.79 \times 10^{-6}$ & $5.07 \times 10^{-3}$ & $1.83 \times 10^{-3}$ & $1.74 \times 10^{-3}$ \\
\hline No. & {$[\mathrm{OH}] /$} & $k_{1 \Psi, \mathrm{obs}} /$ & $k_{1 \Psi, \mathrm{OH}-} /$ & $k_{1 \Psi} /$ \\
\hline BUT-191-1 & $4.72 \times 10^{-3}$ & $1.40 \times 10^{-1}$ & $1.11 \times 10^{-1}$ & $2.90 \times 10^{-2}$ \\
BUT-191-2 & $4.54 \times 10^{-3}$ & $1.53 \times 10^{-1}$ & $1.07 \times 10^{-1}$ & $4.58 \times 10^{-2}$ \\
BUT-191-3 & $4.37 \times 10^{-3}$ & $1.64 \times 10^{-1}$ & $1.03 \times 10^{-1}$ & $6.14 \times 10^{-2}$ \\
BUT-191-4 & $4.19 \times 10^{-3}$ & $1.76 \times 10^{-1}$ & $9.89 \times 10^{-2}$ & $7.73 \times 10^{-2}$ \\
BUT-191-5 & $3.76 \times 10^{-3}$ & $2.11 \times 10^{-1}$ & $8.87 \times 10^{-2}$ & $1.22 \times 10^{-1}$ \\
BUT-191-6 & $3.33 \times 10^{-3}$ & $2.43 \times 10^{-1}$ & $7.85 \times 10^{-2}$ & $1.64 \times 10^{-1}$ \\
\hline
\end{tabular}

${ }^{a}$ Calculated with $k_{2, \mathrm{OH}^{-}}=2.36 \times 10^{1} \mathrm{~L} \mathrm{~mol}^{-1} \mathrm{~s}^{-1}$ from ref 11 .

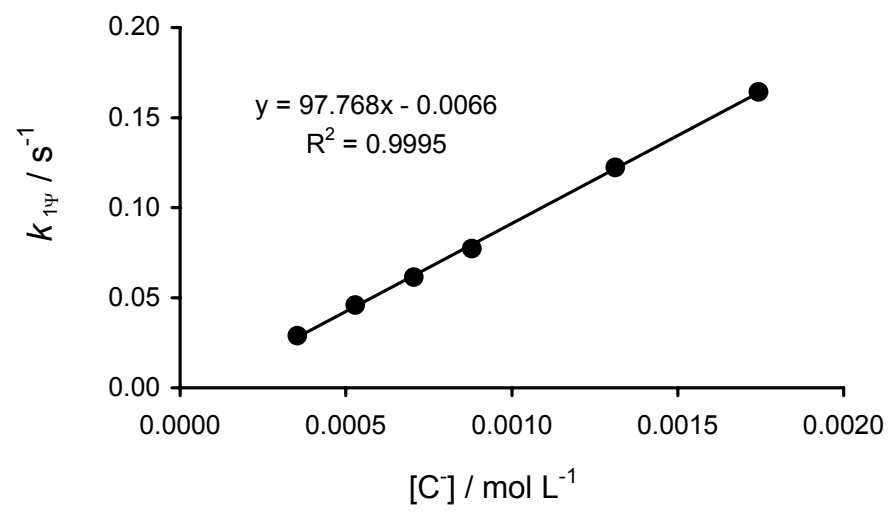

$k_{2, \mathrm{C}^{-}}=9.78 \times 10^{1} \mathrm{~L} \mathrm{~mol}^{-1} \mathrm{~s}^{-1}$ 
Table S5. Potassium salt of nitromethide (1a) and $2 \mathbf{c}$ at $630 \mathrm{~nm}$ at $20{ }^{\circ} \mathrm{C}$ in water (J\&M).

\begin{tabular}{ccccc}
\hline No. & $\begin{array}{c}{[\mathbf{2 c}]_{0} /} \\
\mathrm{mol} \mathrm{L}^{-1}\end{array}$ & $\begin{array}{c}{\left[\mathrm{OH}^{-}\right]_{0} /} \\
\mathrm{mol} \mathrm{L}^{-1}\end{array}$ & $\begin{array}{c}{[\mathbf{1 a - H}]_{0} /} \\
\mathrm{mol} \mathrm{L}^{-1}\end{array}$ & $\begin{array}{c}{[\mathbf{1 a}] /} \\
\mathrm{mol} \mathrm{L}^{-1}\end{array}$ \\
\hline BUT-192-1 & $2.21 \times 10^{-5}$ & $1.65 \times 10^{-4}$ & $8.95 \times 10^{-4}$ & $1.35 \times 10^{-4}$ \\
BUT-192-2 & $2.19 \times 10^{-5}$ & $4.07 \times 10^{-4}$ & $2.21 \times 10^{-3}$ & $3.73 \times 10^{-4}$ \\
BUT-192-3 & $1.86 \times 10^{-5}$ & $6.93 \times 10^{-4}$ & $3.76 \times 10^{-3}$ & $6.58 \times 10^{-4}$ \\
BUT-192-5 & $2.00 \times 10^{-5}$ & $1.87 \times 10^{-3}$ & $1.21 \times 10^{-2}$ & $1.84 \times 10^{-3}$ \\
\hline No. & $k_{1 \Psi, \text { obs }} /$ & Conv./ & $k_{1 \Psi, \mathrm{OH}^{-}} /$ & $k_{1 \Psi} /$ \\
$\mathrm{s}^{-1}$ & $\%$ & $\mathrm{~s}^{-1, a}$ & $\mathrm{~s}^{-1}$ \\
\hline BUT-192-1 & $1.74 \times 10^{-3}$ & 35 & $6.39 \times 10^{-5}$ & $1.68 \times 10^{-3}$ \\
BUT-192-2 & $5.03 \times 10^{-3}$ & 83 & $7.29 \times 10^{-5}$ & $4.96 \times 10^{-3}$ \\
BUT-192-3 & $8.21 \times 10^{-3}$ & 69 & $7.60 \times 10^{-5}$ & $8.13 \times 10^{-3}$ \\
BUT-192-5 & $2.29 \times 10^{-2}$ & 92 & $6.43 \times 10^{-5}$ & $2.28 \times 10^{-2}$ \\
\hline Calculed & & & &
\end{tabular}

${ }^{a}$ Calculated with $k_{2, \mathrm{OH}^{-}}=2.16 \mathrm{~L} \mathrm{~mol}^{-1} \mathrm{~s}^{-1}$ from ref 11 .

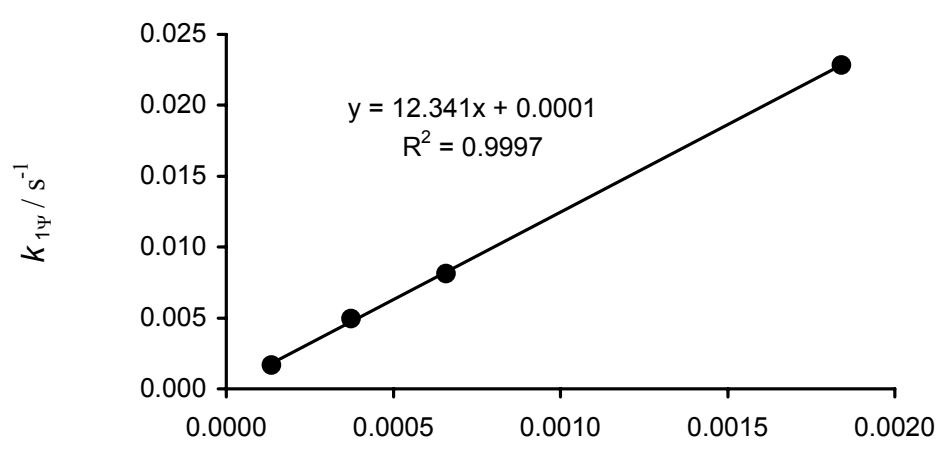

$\left[C^{-}\right] / \mathrm{mol} \mathrm{L}^{-1}$

$k_{2, \mathrm{C}^{-}}=1.23 \times 10^{1} \mathrm{~L} \mathrm{~mol}^{-1} \mathrm{~s}^{-1}$ 
Table S6. Potassium salt of nitroethanide (1b), 2a, and benzenesulfonic acid (BSA) at $605 \mathrm{~nm}$ at $20{ }^{\circ} \mathrm{C}$ in water $(\mathrm{J} \& \mathrm{M})$.

\begin{tabular}{rccccc}
\hline \multicolumn{1}{c}{ No. } & $\begin{array}{c}{[\mathbf{2 a}]_{0} /} \\
\mathrm{mol} \mathrm{L}^{-1}\end{array}$ & $\begin{array}{c}{[\mathrm{BSA}] /} \\
\mathrm{mol} \mathrm{L}^{-1}\end{array}$ & $\begin{array}{c}{\left[\mathrm{OH}^{-}\right]_{0} /} \\
\mathrm{mol} \mathrm{L}^{-1, a}\end{array}$ & $\begin{array}{c}{[\mathbf{1 b}-\mathbf{H}]_{0} /} \\
\mathrm{mol} \mathrm{L}^{-1}\end{array}$ & $\begin{array}{c}{[\mathbf{1 b}] /} \\
\mathrm{mol} \mathrm{L}^{-1}\end{array}$ \\
\hline BUT-203-2 & $2.26 \times 10^{-5}$ & $2.29 \times 10^{-5}$ & $9.21 \times 10^{-5}$ & $1.65 \times 10^{-4}$ & $8.76 \times 10^{-5}$ \\
BUT-203-1 & $2.49 \times 10^{-5}$ & $2.52 \times 10^{-5}$ & $3.97 \times 10^{-4}$ & $6.04 \times 10^{-4}$ & $3.90 \times 10^{-4}$ \\
BUT-203-3 & $2.25 \times 10^{-5}$ & $2.28 \times 10^{-5}$ & $5.50 \times 10^{-4}$ & $1.09 \times 10^{-3}$ & $5.46 \times 10^{-4}$ \\
BUT-203-4 & $2.81 \times 10^{-5}$ & $2.84 \times 10^{-5}$ & $9.25 \times 10^{-4}$ & $2.04 \times 10^{-3}$ & $9.21 \times 10^{-4}$ \\
BUT-203-5 & $2.58 \times 10^{-5}$ & $2.62 \times 10^{-5}$ & $1.07 \times 10^{-3}$ & $2.51 \times 10^{-3}$ & $1.07 \times 10^{-3}$ \\
\hline No. & {$\left[\mathrm{OH}^{-}\right] /$} & $k_{1 \Psi, \mathrm{obs}} /$ & $\mathrm{Conv} . /$ & $k_{1 \Psi, \mathrm{OH}^{-} /}$ & $k_{1 \Psi} /$ \\
\hline BUT-203-2 & $4.51 \times 10^{-6}$ & $3.10 \times 10^{-2}$ & 85 & $3.59 \times 10^{-3}$ & $2.74 \times 10^{-2}$ \\
BUT-203-1 & $7.23 \times 10^{-6}$ & $7.93 \times 10^{-2}$ & 91 & $4.25 \times 10^{-3}$ & $7.51 \times 10^{-2}$ \\
BUT-203-3 & $4.00 \times 10^{-6}$ & $1.05 \times 10^{-1}$ & 94 & $3.51 \times 10^{-3}$ & $1.01 \times 10^{-1}$ \\
BUT-203-4 & $3.28 \times 10^{-6}$ & $1.66 \times 10^{-1}$ & 86 & $4.15 \times 10^{-3}$ & $1.62 \times 10^{-1}$ \\
BUT-203-5 & $2.96 \times 10^{-6}$ & $1.94 \times 10^{-1}$ & 88 & $3.82 \times 10^{-3}$ & $1.90 \times 10^{-1}$ \\
\hline
\end{tabular}

${ }^{a}$ Remaining $\left[\mathrm{OH}^{-}\right]_{0}$ after neutralization by benzenesulfonic acid (BSA).

${ }^{b}$ Calculated with $k_{2, \mathrm{OH}^{-}}=1.31 \times 10^{2} \mathrm{~L} \mathrm{~mol}^{-1} \mathrm{~s}^{-1}$ from ref 11 .

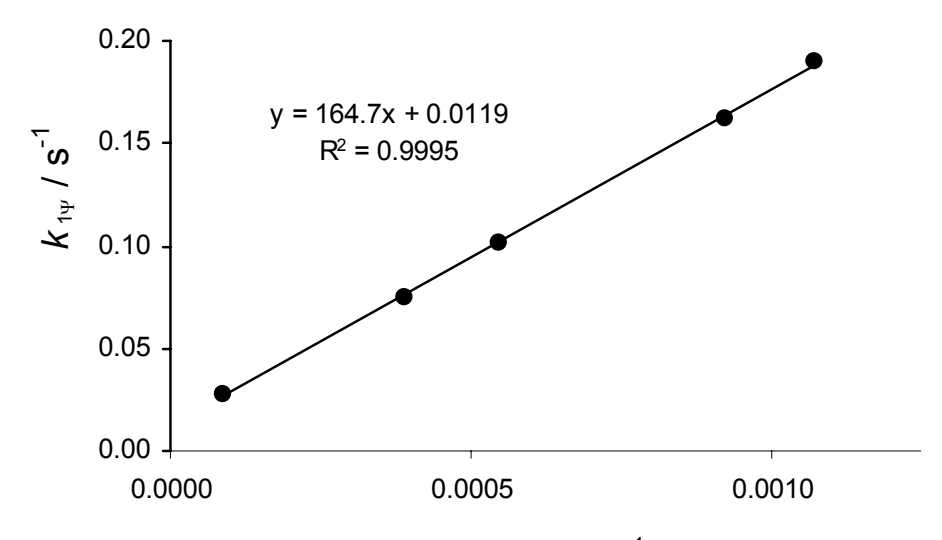

$k_{2, \mathrm{C}^{-}}=1.65 \times 10^{2} \mathrm{~L} \mathrm{~mol}^{-1} \mathrm{~s}^{-1}$ 
Table S7. Potassium salt of nitroethanide (1b) and $\mathbf{2 b}$ at $620 \mathrm{~nm}$ at $20{ }^{\circ} \mathrm{C}$ in water (J\&M).

\begin{tabular}{|c|c|c|c|c|c|}
\hline No. & $\begin{array}{c}{[\mathbf{2} \mathbf{b}]_{0} /} \\
\mathrm{mol} \mathrm{L}^{-1}\end{array}$ & $\begin{array}{l}{\left[\mathrm{OH}^{-}\right]_{0} /} \\
\mathrm{mol} \mathrm{L}^{-1}\end{array}$ & $\begin{array}{c}{[\mathbf{1 b}-\mathbf{H}]_{0} /} \\
\mathrm{mol} \mathrm{L}^{-1}\end{array}$ & $\begin{array}{c}{[\mathbf{1 b}] /} \\
\mathrm{mol} \mathrm{L}^{-1}\end{array}$ & $\begin{array}{l}{\left[\mathrm{OH}^{-}\right] /} \\
\mathrm{mol} \mathrm{L}^{-1}\end{array}$ \\
\hline BUT-202-2 & $1.44 \times 10^{-5}$ & $2.74 \times 10^{-4}$ & $5.43 \times 10^{-4}$ & $2.70 \times 10^{-4}$ & $3.94 \times 10^{-6}$ \\
\hline BUT-202-3 & $1.43 \times 10^{-5}$ & $5.86 \times 10^{-4}$ & $1.16 \times 10^{-3}$ & $5.82 \times 10^{-4}$ & $4.01 \times 10^{-6}$ \\
\hline BUT-202-4 & $1.45 \times 10^{-5}$ & $7.91 \times 10^{-4}$ & $1.57 \times 10^{-3}$ & $7.87 \times 10^{-4}$ & $4.00 \times 10^{-6}$ \\
\hline BUT-202-5 & $1.43 \times 10^{-5}$ & $9.73 \times 10^{-3}$ & $1.93 \times 10^{-3}$ & $9.69 \times 10^{-4}$ & $4.01 \times 10^{-6}$ \\
\hline No. & $\begin{array}{c}k_{1 \Psi, \text { obs }} / \\
\mathrm{s}^{-1} \\
\end{array}$ & $\begin{array}{c}\text { Conv. / } \\
\% \\
\end{array}$ & $\begin{array}{c}k_{1 \Psi, \mathrm{OH}^{-}} / \\
\mathrm{s}^{-1, a} \\
\end{array}$ & $\begin{array}{c}k_{1 \Psi} / \\
\mathrm{s}^{-1} \\
\end{array}$ & \\
\hline BUT-202-2 & $1.09 \times 10^{-2}$ & 66 & $9.30 \times 10^{-5}$ & $1.08 \times 10^{-2}$ & \\
\hline BUT-202-3 & $2.12 \times 10^{-2}$ & 80 & $9.46 \times 10^{-5}$ & $2.11 \times 10^{-2}$ & \\
\hline BUT-202-4 & $2.67 \times 10^{-2}$ & 85 & $9.44 \times 10^{-5}$ & $2.66 \times 10^{-2}$ & \\
\hline BUT-202-5 & $3.34 \times 10^{-2}$ & 91 & $9.47 \times 10^{-5}$ & $3.33 \times 10^{-2}$ & \\
\hline
\end{tabular}

${ }^{a}$ Calculated with $k_{2, \mathrm{OH}^{-}}=2.36 \times 10^{1} \mathrm{~L} \mathrm{~mol}^{-1} \mathrm{~s}^{-1}$ from ref 11 .

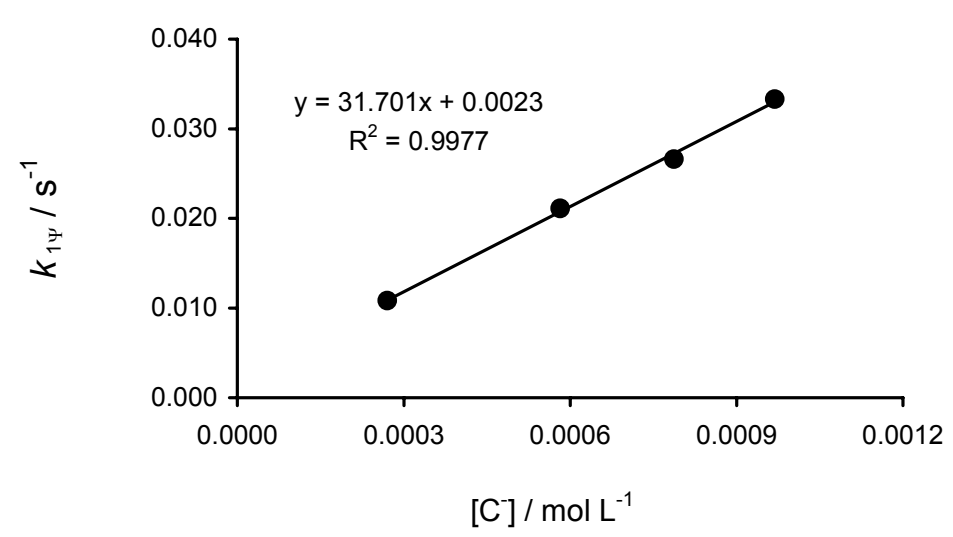

$k_{2, \mathrm{C}^{-}}=3.17 \times 10^{1} \mathrm{~L} \mathrm{~mol}^{-1} \mathrm{~s}^{-1}$ 
Table S8. Potassium salt of nitroethanide (1b) and $\mathbf{2 c}$ at $630 \mathrm{~nm}$ at $20{ }^{\circ} \mathrm{C}$ in water $(\mathrm{J} \& \mathrm{M})$.

\begin{tabular}{rcccc}
\hline \multicolumn{1}{c}{ No. } & $\begin{array}{c}{[\mathbf{2 c}]_{0} /} \\
\mathrm{mol} \mathrm{L}^{-1}\end{array}$ & $\begin{array}{c}{\left[\mathrm{OH}^{-}\right]_{0} /} \\
\mathrm{mol} \mathrm{L}^{-1}\end{array}$ & $\begin{array}{c}{[\mathbf{1 b}-\mathbf{H}]_{0} /} \\
\mathrm{mol} \mathrm{L}^{-1}\end{array}$ & $\begin{array}{c}{[\mathbf{1 b}] /} \\
\mathrm{mol} \mathrm{L}^{-1}\end{array}$ \\
\hline BUT-201-1 & $2.31 \times 10^{-5}$ & $4.25 \times 10^{-4}$ & $6.02 \times 10^{-4}$ & $4.16 \times 10^{-4}$ \\
BUT-201-2 & $1.53 \times 10^{-5}$ & $6.32 \times 10^{-4}$ & $1.19 \times 10^{-3}$ & $6.28 \times 10^{-4}$ \\
BUT-201-3 & $1.56 \times 10^{-5}$ & $1.29 \times 10^{-3}$ & $2.43 \times 10^{-3}$ & $1.29 \times 10^{-3}$ \\
BUT-201-4 & $1.45 \times 10^{-5}$ & $1.60 \times 10^{-3}$ & $3.40 \times 10^{-3}$ & $1.60 \times 10^{-3}$ \\
BUT-201-6 & $1.48 \times 10^{-5}$ & $2.04 \times 10^{-3}$ & $4.63 \times 10^{-3}$ & $2.04 \times 10^{-3}$ \\
\hline No. & $k_{1 \Psi, \text { obs } /}$ & $\mathrm{Conv} . /$ & $k_{1 \Psi, \mathrm{OH}^{-} /}$ & $k_{1 \Psi} /$ \\
\hline BUT-201-1 & $1.48 \times 10^{-3}$ & 62 & $1.92 \times 10^{-5}$ & $1.46 \times 10^{-3}$ \\
BUT-201-2 & $2.73 \times 10^{-3}$ & 73 & $9.59 \times 10^{-6}$ & $2.72 \times 10^{-3}$ \\
BUT-201-3 & $5.55 \times 10^{-3}$ & 83 & $9.66 \times 10^{-6}$ & $5.54 \times 10^{-3}$ \\
BUT-201-4 & $7.09 \times 10^{-3}$ & 80 & $7.61 \times 10^{-6}$ & $7.08 \times 10^{-3}$ \\
BUT-201-6 & $8.66 \times 10^{-3}$ & 71 & $6.75 \times 10^{-6}$ & $8.65 \times 10^{-3}$ \\
\hline
\end{tabular}

${ }^{a}$ Calculated with $k_{2, \mathrm{OH}^{-}}=2.16 \mathrm{~L} \mathrm{~mol}^{-1} \mathrm{~s}^{-1}$ from ref 11 .

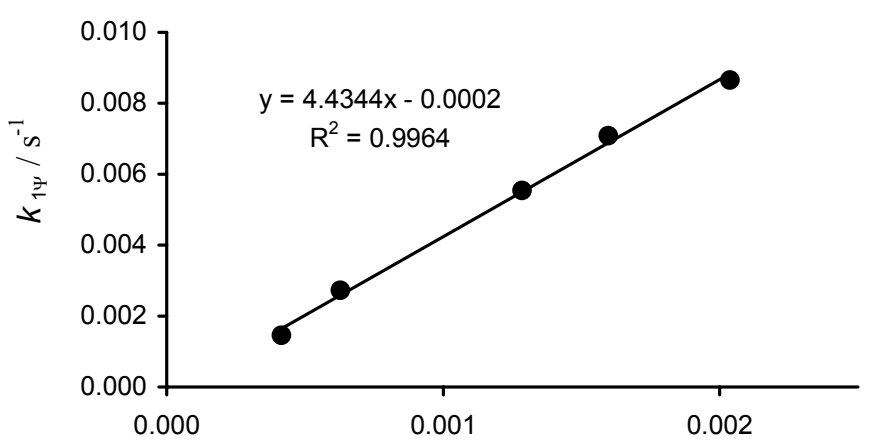

$\left[\mathrm{C}^{-}\right] / \mathrm{mol} \mathrm{L}^{-1}$

$k_{2, \mathrm{C}^{-}}=4.43 \mathrm{~L} \mathrm{~mol}^{-1} \mathrm{~s}^{-1}$ 
Table S9. Potassium salt of 2-nitropropanide (1c), 2a, and benzenesulfonic acid (BSA) at $605 \mathrm{~nm}$ at $20{ }^{\circ} \mathrm{C}$ in water $(\mathrm{J} \& \mathrm{M})$.

\begin{tabular}{cccccc}
\hline \multicolumn{1}{c}{ No. } & $\begin{array}{c}{[\mathbf{2 a}]_{0} /} \\
\mathrm{mol} \mathrm{L}^{-1}\end{array}$ & $\begin{array}{c}{[\mathrm{BSA}] /} \\
\mathrm{mol} \mathrm{L}\end{array}$ & $\begin{array}{c}{\left[\mathrm{OH}^{-1}\right]_{0} /} \\
\mathrm{mol} \mathrm{L}^{-1, a}\end{array}$ & $\begin{array}{c}{[\mathbf{1 c - H}]_{0} /} \\
\mathrm{mol} \mathrm{L}^{-1}\end{array}$ & $\begin{array}{c}{[\mathbf{1 c}] /} \\
\mathrm{mol} \mathrm{L}^{-1}\end{array}$ \\
\hline BUT-205-4 & $1.69 \times 10^{-5}$ & $1.72 \times 10^{-5}$ & $1.55 \times 10^{-4}$ & $2.59 \times 10^{-4}$ & $1.54 \times 10^{-4}$ \\
BUT-205-3 & $1.66 \times 10^{-5}$ & $2.52 \times 10^{-5}$ & $3.12 \times 10^{-4}$ & $4.23 \times 10^{-3}$ & $3.12 \times 10^{-4}$ \\
BUT-205-2 & $3.21 \times 10^{-5}$ & $3.26 \times 10^{-5}$ & $6.61 \times 10^{-4}$ & $8.20 \times 10^{-4}$ & $6.59 \times 10^{-4}$ \\
BUT-205-1 & $2.97 \times 10^{-5}$ & $3.02 \times 10^{-5}$ & $1.29 \times 10^{-3}$ & $1.52 \times 10^{-3}$ & $1.29 \times 10^{-3}$ \\
\hline No. & {$\left[\mathrm{OH}^{-}\right] /$} & $k_{1 \Psi, \mathrm{obs}} /$ & Conv. / & $k_{1 \Psi, \mathrm{OH}^{-} /}$ & $k_{1 \Psi} /$ \\
\hline BUT-205-4 & $8.06 \times 10^{-7}$ & $4.30 \times 10^{-2}$ & 91 & $1.06 \times 10^{-4}$ & $4.29 \times 10^{-2}$ \\
BUT-205-3 & $4.37 \times 10^{-8}$ & $6.44 \times 10^{-2}$ & 92 & $5.73 \times 10^{-6}$ & $6.44 \times 10^{-2}$ \\
BUT-205-2 & $2.25 \times 10^{-6}$ & $1.08 \times 10^{-1}$ & 72 & $2.95 \times 10^{-4}$ & $1.08 \times 10^{-1}$ \\
BUT-205-1 & $3.03 \times 10^{-6}$ & $1.81 \times 10^{-1}$ & 82 & $3.97 \times 10^{-4}$ & $1.81 \times 10^{-1}$ \\
\hline
\end{tabular}

${ }^{a}$ Remaining $\left[\mathrm{OH}^{-}\right]_{0}$ after neutralization by benzenesulfonic acid (BSA).

${ }^{b}$ Calculated with $k_{2, \mathrm{OH}^{-}}=1.31 \times 10^{2} \mathrm{~L} \mathrm{~mol}^{-1} \mathrm{~s}^{-1}$ from ref 11 .

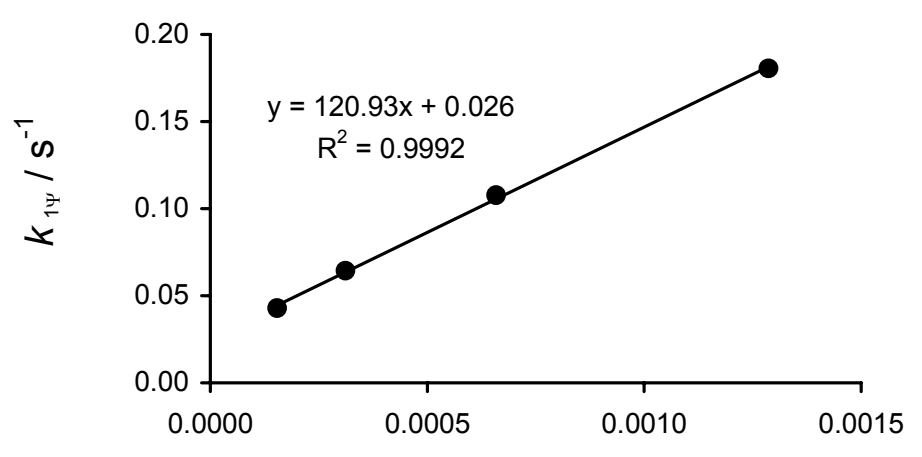

$\left[\mathrm{C}^{-}\right] / \mathrm{mol} \mathrm{L}^{-1}$

$k_{2, \mathrm{C}^{-}}=1.21 \times 10^{2} \mathrm{~L} \mathrm{~mol}^{-1} \mathrm{~s}^{-1}$ 
Table S10. Potassium salt of 2-nitropropanide (1c) and $\mathbf{2 b}$ at $620 \mathrm{~nm}$ at $20{ }^{\circ} \mathrm{C}$ in water (J\&M).

\begin{tabular}{rcccc}
\hline \multicolumn{1}{c}{ No. } & $\begin{array}{c}{[\mathbf{2 b}]_{0} /} \\
\mathrm{mol} \mathrm{L}^{-1}\end{array}$ & $\begin{array}{c}{\left[\mathrm{OH}^{-}\right]_{0} /} \\
\mathrm{mol} \mathrm{L}^{-1}\end{array}$ & $\begin{array}{c}{[\mathbf{1 c - H}]_{0} /} \\
\mathrm{mol} \mathrm{L}^{-1}\end{array}$ & $\begin{array}{c}{[\mathbf{1 c}] /} \\
\mathrm{mol} \mathrm{L}^{-1}\end{array}$ \\
\hline BUT-204-1 & $1.61 \times 10^{-5}$ & $3.07 \times 10^{-4}$ & $3.14 \times 10^{-4}$ & $2.97 \times 10^{-4}$ \\
BUT-204-2 & $1.64 \times 10^{-5}$ & $6.24 \times 10^{-4}$ & $6.38 \times 10^{-4}$ & $6.11 \times 10^{-4}$ \\
BUT-204-3 & $1.76 \times 10^{-5}$ & $1.20 \times 10^{-3}$ & $1.37 \times 10^{-3}$ & $1.20 \times 10^{-3}$ \\
BUT-204-4 & $1.69 \times 10^{-5}$ & $1.84 \times 10^{-3}$ & $1.97 \times 10^{-3}$ & $1.83 \times 10^{-3}$ \\
BUT-204-5 & $1.62 \times 10^{-5}$ & $2.20 \times 10^{-3}$ & $2.36 \times 10^{-3}$ & $2.19 \times 10^{-3}$ \\
\hline No. & $k_{1 \Psi, \text { obs }} /$ & $\mathrm{Conv.} \mathrm{/}$ & $k_{1 \Psi, \mathrm{OH}^{-} /}$ & $k_{1 \Psi} /$ \\
\hline BUT-204-1 & $1.21 \times 10^{-2}$ & 55 & $2.30 \times 10^{-4}$ & $1.19 \times 10^{-2}$ \\
BUT-204-2 & $1.86 \times 10^{-2}$ & 78 & $2.98 \times 10^{-4}$ & $1.83 \times 10^{-2}$ \\
BUT-204-3 & $3.12 \times 10^{-2}$ & 92 & $8.92 \times 10^{-5}$ & $3.11 \times 10^{-2}$ \\
BUT-204-4 & $4.62 \times 10^{-2}$ & 95 & $1.73 \times 10^{-4}$ & $4.60 \times 10^{-2}$ \\
BUT-204-5 & $5.30 \times 10^{-2}$ & 93 & $1.70 \times 10^{-4}$ & $5.28 \times 10^{-2}$ \\
\hline
\end{tabular}

${ }^{a}$ Calculated with $k_{2, \mathrm{OH}^{-}}=2.36 \times 10^{1} \mathrm{~L} \mathrm{~mol}^{-1} \mathrm{~s}^{-1}$ from ref 11 .

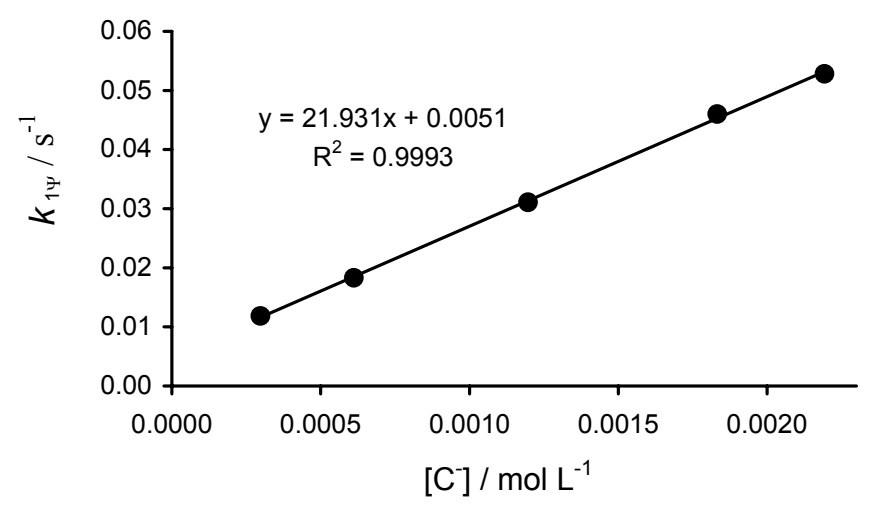

$k_{2, \mathrm{C}^{-}}=2.19 \times 10^{1} \mathrm{~L} \mathrm{~mol}^{-1} \mathrm{~s}^{-1}$ 
Table S11. Potassium salt of 2-nitropropanide (1c) and $\mathbf{2 c}$ at $630 \mathrm{~nm}$ at $20{ }^{\circ} \mathrm{C}$ in water (J\&M).

\begin{tabular}{rcccc}
\hline \multicolumn{1}{c}{ No. } & $\begin{array}{c}{[\mathbf{2 c}]_{0} /} \\
\mathrm{mol} \mathrm{L}^{-1}\end{array}$ & $\begin{array}{c}{\left[\mathrm{OH}^{-}\right]_{0} /} \\
\mathrm{mol} \mathrm{L}^{-1}\end{array}$ & $\begin{array}{c}{[\mathbf{1 c - H}]_{0} /} \\
\mathrm{mol} \mathrm{L}^{-1}\end{array}$ & $\begin{array}{c}{[\mathbf{1 c}] /} \\
\mathrm{mol} \mathrm{L}^{-1}\end{array}$ \\
\hline BUT-200-2 & $1.54 \times 10^{-5}$ & $2.98 \times 10^{-4}$ & $3.71 \times 10^{-4}$ & $2.96 \times 10^{-4}$ \\
BUT-200-3 & $1.49 \times 10^{-5}$ & $6.13 \times 10^{-4}$ & $7.06 \times 10^{-4}$ & $6.10 \times 10^{-4}$ \\
BUT-200-4 & $1.50 \times 10^{-5}$ & $8.25 \times 10^{-4}$ & $1.07 \times 10^{-3}$ & $8.23 \times 10^{-4}$ \\
BUT-200-5 & $1.55 \times 10^{-5}$ & $1.70 \times 10^{-3}$ & $2.20 \times 10^{-3}$ & $1.70 \times 10^{-3}$ \\
BUT-200-6 & $1.51 \times 10^{-5}$ & $2.07 \times 10^{-3}$ & $2.86 \times 10^{-3}$ & $2.07 \times 10^{-3}$ \\
\hline No. & $k_{1 \Psi, \text { obs } /}$ & $\mathrm{Conv} . /$ & $k_{1 \Psi, \mathrm{OH}^{-} /}$ & $k_{1 \Psi} /$ \\
\hline BUT-200-2 & $9.87 \times 10^{-4}$ & 33 & $4.67 \times 10^{-6}$ & $9.82 \times 10^{-4}$ \\
BUT-200-3 & $2.05 \times 10^{-3}$ & 69 & $7.50 \times 10^{-6}$ & $2.04 \times 10^{-3}$ \\
BUT-200-4 & $2.61 \times 10^{-3}$ & 75 & $3.96 \times 10^{-6}$ & $2.61 \times 10^{-3}$ \\
BUT-200-5 & $4.37 \times 10^{-3}$ & 71 & $4.02 \times 10^{-6}$ & $4.37 \times 10^{-3}$ \\
BUT-200-6 & $5.50 \times 10^{-3}$ & 76 & $3.10 \times 10^{-6}$ & $5.50 \times 10^{-3}$ \\
\hline
\end{tabular}

${ }^{a}$ Calculated with $k_{2, \mathrm{OH}^{-}}=2.16 \mathrm{~L} \mathrm{~mol}^{-1} \mathrm{~s}^{-1}$ from ref 11 .

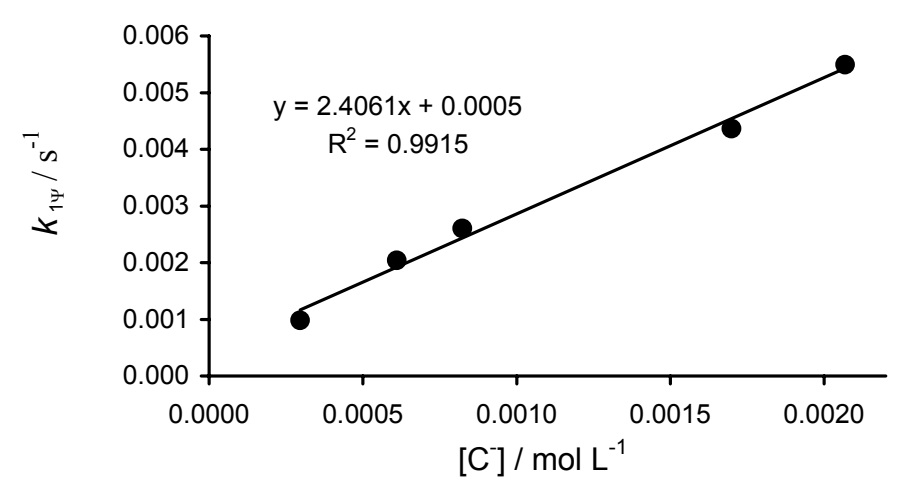

$k_{2, \mathrm{C}^{-}}=2.41 \mathrm{~L} \mathrm{~mol}^{-1} \mathrm{~s}^{-1}$ 
Table S12. Potassium salt of phenylnitromethanide (1d), 2a, and benzenesulfonic acid (BSA) at $605 \mathrm{~nm}$ at $20{ }^{\circ} \mathrm{C}$ in water (stopped-flow).

\begin{tabular}{|c|c|c|c|c|c|}
\hline No. & $\begin{array}{c}{[\mathbf{2 a}]_{0} /} \\
\mathrm{mol} \mathrm{L}^{-1}\end{array}$ & $\begin{array}{l}{[\mathrm{BSA}] /} \\
\mathrm{mol} \mathrm{L}^{-1}\end{array}$ & $\begin{array}{l}{\left[\mathrm{OH}^{-}\right]_{0} /} \\
\mathrm{mol} \mathrm{L}^{-1, a}\end{array}$ & $\begin{array}{c}{[\mathbf{1 d}-\mathbf{H}]_{0} /} \\
\mathrm{mol} \mathrm{L}^{-1}\end{array}$ & $\begin{array}{l}{\left[\mathrm{OH}^{-}\right] /} \\
\mathrm{mol} \mathrm{L}^{-1}\end{array}$ \\
\hline BUT-227-1 & $9.82 \times 10^{-6}$ & $1.04 \times 10^{-5}$ & $4.90 \times 10^{-4}$ & $4.06 \times 10^{-4}$ & $8.39 \times 10^{-5}$ \\
\hline BUT-227-2 & $9.82 \times 10^{-6}$ & $1.04 \times 10^{-5}$ & $9.90 \times 10^{-4}$ & $8.12 \times 10^{-4}$ & $1.78 \times 10^{-4}$ \\
\hline BUT-227-3 & $9.82 \times 10^{-6}$ & $1.04 \times 10^{-5}$ & $1.99 \times 10^{-3}$ & $1.62 \times 10^{-3}$ & $3.70 \times 10^{-4}$ \\
\hline BUT-227-4 & $9.82 \times 10^{-6}$ & $1.04 \times 10^{-5}$ & $3.99 \times 10^{-3}$ & $3.25 \times 10^{-3}$ & $7.40 \times 10^{-4}$ \\
\hline No. & $\begin{array}{c}{[\mathbf{1 d}] /} \\
\mathrm{mol} \mathrm{L}^{-1}\end{array}$ & $\begin{array}{c}k_{1 \Psi, \mathrm{obs}} / \\
\mathrm{s}^{-1}\end{array}$ & $\begin{array}{c}k_{1 \Psi, \mathrm{OH}^{-}} / \\
\mathrm{s}^{-1, b}\end{array}$ & $\begin{array}{c}k_{1 \Psi} / \\
\mathrm{s}^{-1} \\
\end{array}$ & \\
\hline BUT-227-1 & $4.06 \times 10^{-4}$ & $1.94 \times 10^{-1}$ & $1.10 \times 10^{-2}$ & $1.83 \times 10^{-1}$ & \\
\hline BUT-227-2 & $8.12 \times 10^{-4}$ & $3.45 \times 10^{-1}$ & $2.33 \times 10^{-2}$ & $3.21 \times 10^{-1}$ & \\
\hline BUT-227-3 & $1.62 \times 10^{-3}$ & $6.99 \times 10^{-1}$ & $4.85 \times 10^{-2}$ & $6.51 \times 10^{-1}$ & \\
\hline BUT-227-4 & $3.25 \times 10^{-3}$ & 1.39 & $9.69 \times 10^{-2}$ & 1.29 & \\
\hline
\end{tabular}

${ }^{a}$ Remaining $\left[\mathrm{OH}^{-}\right]_{0}$ after neutralization by benzenesulfonic acid (BSA).

${ }^{b}$ Calculated with $k_{2, \mathrm{OH}^{-}}=1.31 \times 10^{2} \mathrm{~L} \mathrm{~mol}^{-1} \mathrm{~s}^{-1}$ from ref 11 .

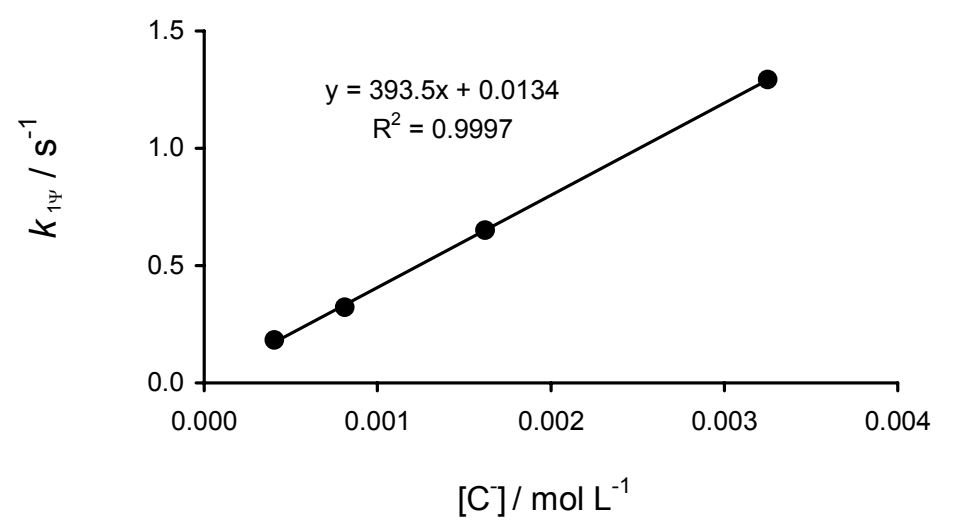

$k_{2, \mathrm{C}^{-}}=3.94 \times 10^{2} \mathrm{~L} \mathrm{~mol}^{-1} \mathrm{~s}^{-1}$ 
Table S13. Potassium salt of phenylnitromethanide (1d) and $\mathbf{2 b}$ at $620 \mathrm{~nm}$ at $20{ }^{\circ} \mathrm{C}$ in water $(\mathrm{J} \& \mathrm{M})$.

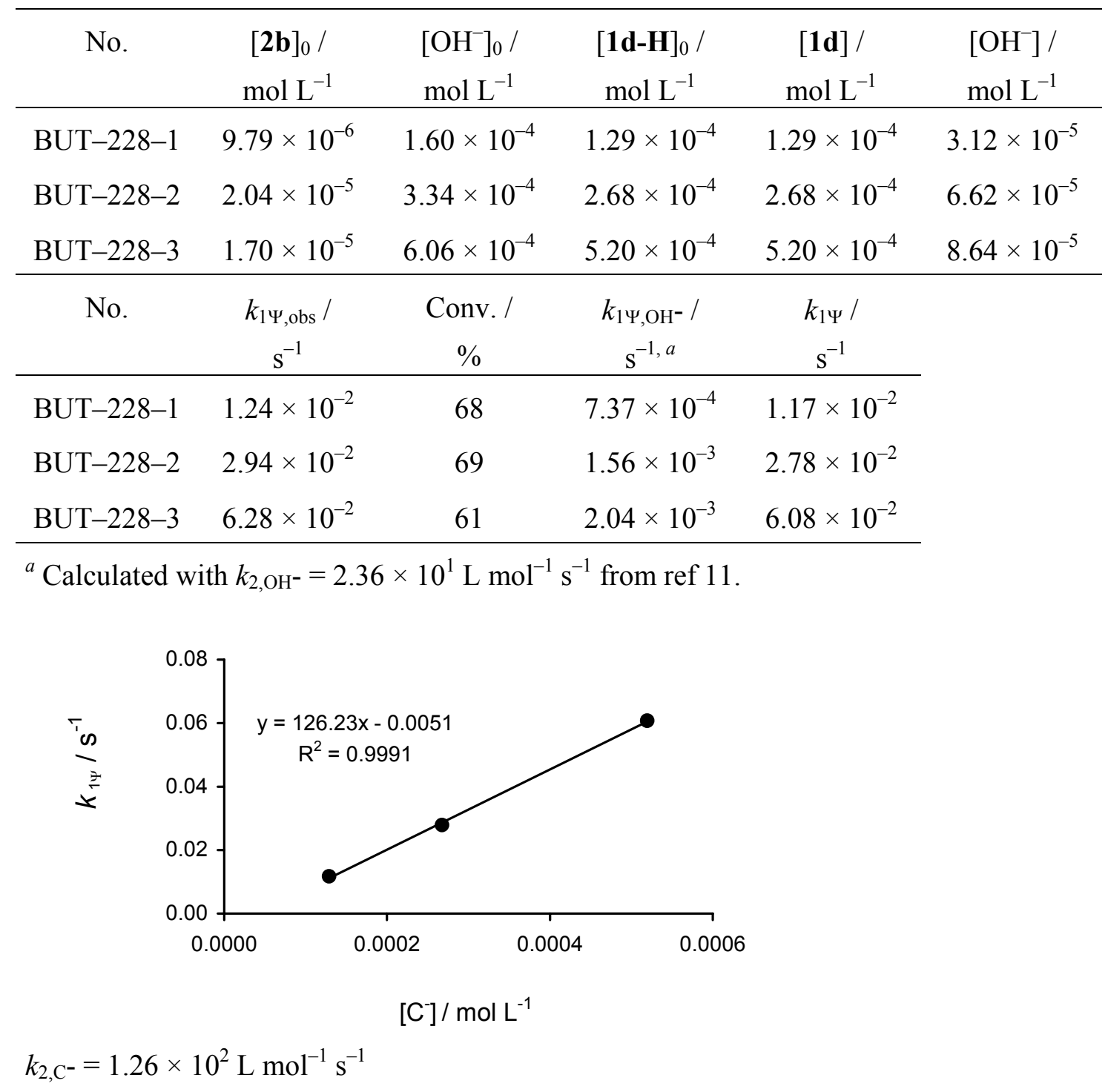


Table S14. Potassium salt of phenylnitromethanide (1d) and $2 \mathrm{c}$ at $630 \mathrm{~nm}$ at $20{ }^{\circ} \mathrm{C}$ in water (J\&M).

\begin{tabular}{|c|c|c|c|c|c|}
\hline No. & $\begin{array}{c}{[\mathbf{2} \mathbf{c}]_{0} /} \\
\mathrm{mol} \mathrm{L}^{-1}\end{array}$ & $\begin{array}{l}{\left[\mathrm{OH}^{-}\right]_{0} /} \\
\mathrm{mol} \mathrm{L}^{-1}\end{array}$ & $\begin{array}{c}{[\mathbf{1 d}-\mathbf{H}]_{0} /} \\
\mathrm{mol} \mathrm{L}^{-1}\end{array}$ & $\begin{array}{c}{[\mathbf{1 d}] /} \\
\mathrm{mol} \mathrm{L}^{-1}\end{array}$ & $\begin{array}{l}{\left[\mathrm{OH}^{-}\right] /} \\
\mathrm{mol} \mathrm{L}^{-1}\end{array}$ \\
\hline BUT-226-4 & $1.52 \times 10^{-5}$ & $1.68 \times 10^{-4}$ & $1.70 \times 10^{-4}$ & $1.65 \times 10^{-4}$ & $2.68 \times 10^{-6}$ \\
\hline BUT-226-1 & $1.47 \times 10^{-5}$ & $3.24 \times 10^{-4}$ & $3.29 \times 10^{-4}$ & $3.21 \times 10^{-4}$ & $3.03 \times 10^{-6}$ \\
\hline BUT-226-2 & $1.28 \times 10^{-5}$ & $5.64 \times 10^{-4}$ & $5.73 \times 10^{-4}$ & $5.61 \times 10^{-4}$ & $3.42 \times 10^{-6}$ \\
\hline BUT-226-3 & $1.35 \times 10^{-5}$ & $1.19 \times 10^{-3}$ & $1.21 \times 10^{-3}$ & $1.19 \times 10^{-3}$ & $3.78 \times 10^{-6}$ \\
\hline No. & $\begin{array}{c}k_{1 \Psi, \mathrm{obs}} / \\
\mathrm{s}^{-1}\end{array}$ & $\begin{array}{c}\text { Conv. / } \\
\% \\
\end{array}$ & $\begin{array}{c}k_{1 \Psi, \mathrm{OH}^{-}} / \\
\mathrm{s}^{-1, a}\end{array}$ & $\begin{array}{c}k_{1 \Psi} / \\
\mathrm{s}^{-1}\end{array}$ & \\
\hline BUT-226-4 & $2.24 \times 10^{-3}$ & 67 & $5.79 \times 10^{-6}$ & $2.23 \times 10^{-3}$ & \\
\hline BUT-226-1 & $3.97 \times 10^{-3}$ & 69 & $6.55 \times 10^{-6}$ & $3.96 \times 10^{-3}$ & \\
\hline BUT-226-2 & $6.13 \times 10^{-3}$ & 82 & $7.39 \times 10^{-6}$ & $6.12 \times 10^{-3}$ & \\
\hline BUT-226-3 & $1.31 \times 10^{-2}$ & 84 & $8.17 \times 10^{-6}$ & $1.31 \times 10^{-2}$ & \\
\hline
\end{tabular}

${ }^{a}$ Calculated with $k_{2, \mathrm{OH}^{-}}=2.16 \mathrm{~L} \mathrm{~mol}^{-1} \mathrm{~s}^{-1}$ from ref 11 .

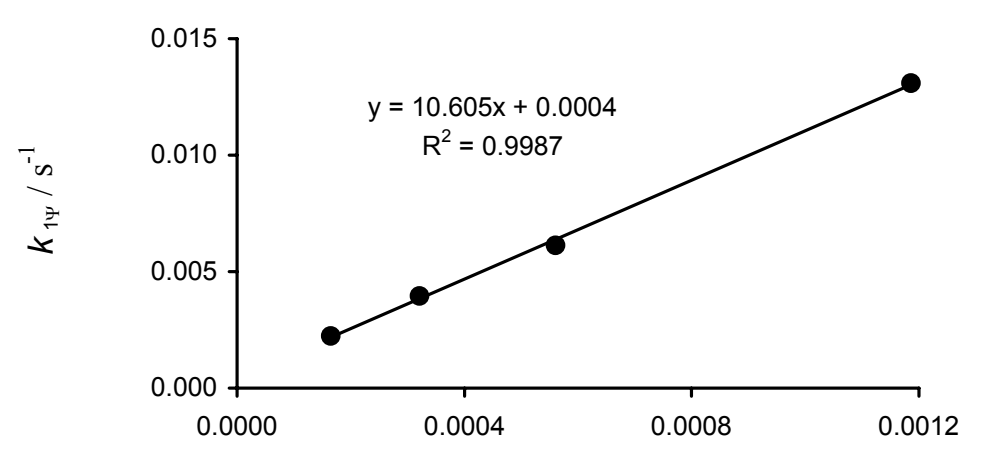

$\left[\mathrm{C}^{-}\right] / \mathrm{mol} \mathrm{L}^{-1}$

$k_{2, \mathrm{C}^{-}}=1.06 \times 10^{1} \mathrm{~L} \mathrm{~mol}^{-1} \mathrm{~s}^{-1}$ 
Table S15. Potassium salt of 4-methylphenylnitromethanide (1e) and $2 \mathbf{a}$ at $605 \mathrm{~nm}$ at $20{ }^{\circ} \mathrm{C}$ in water (J\&M).

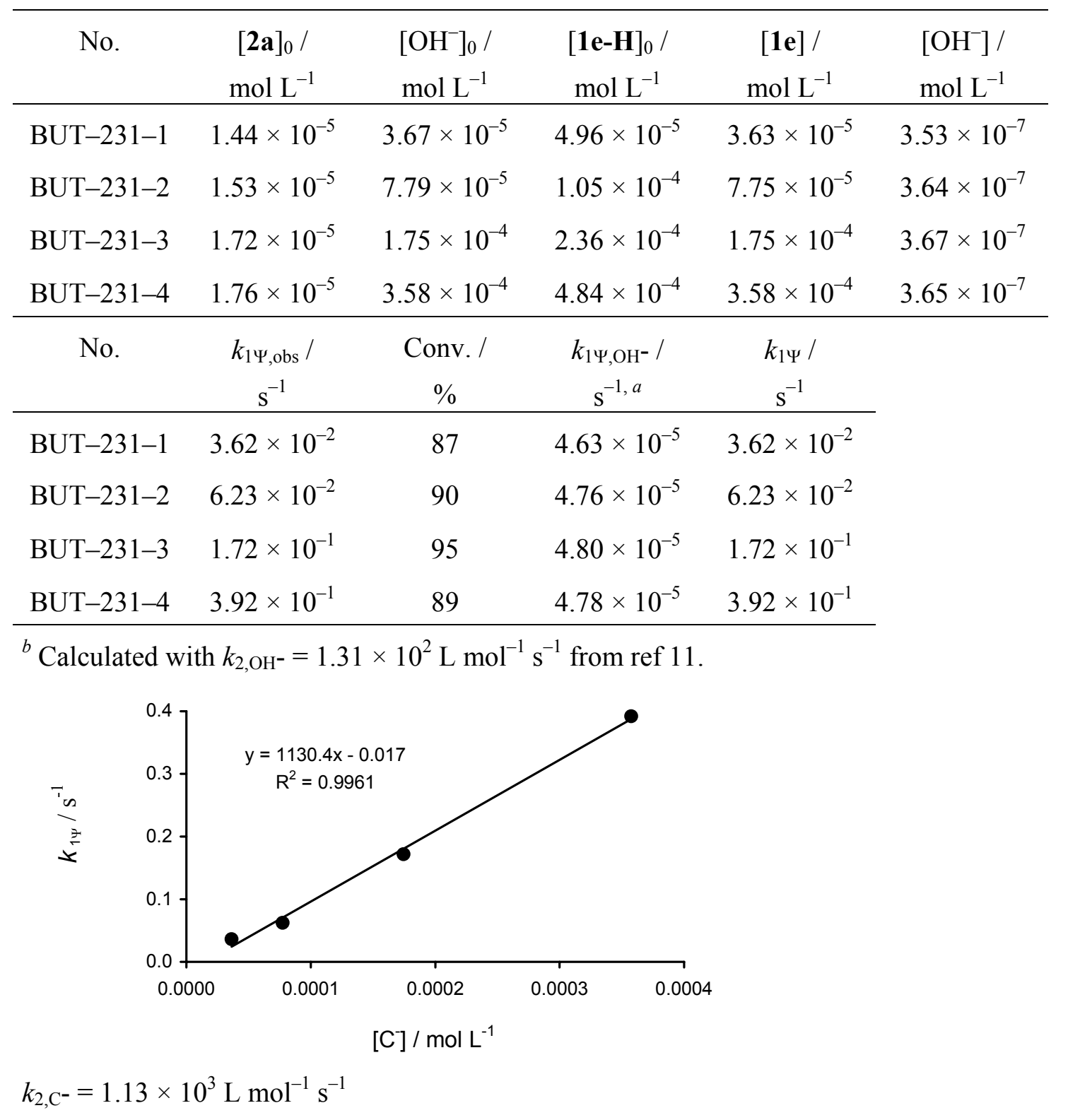


Table S16. Potassium salt of 4-methylphenylnitromethanide (1e) and $\mathbf{2 b}$ at $620 \mathrm{~nm}$ at $20{ }^{\circ} \mathrm{C}$ in water (J\&M).

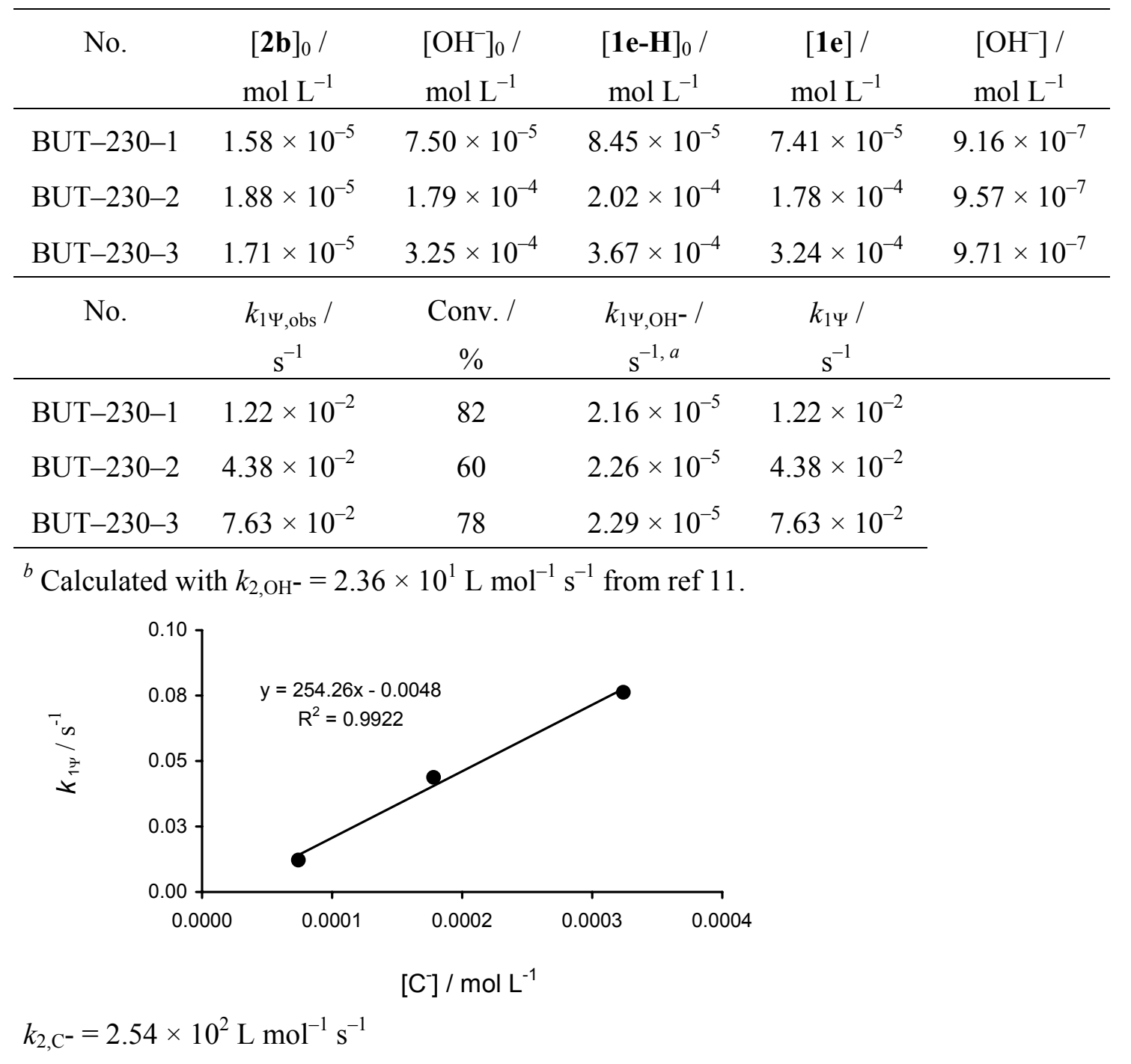


Table S17. Potassium salt of 4-methylphenylnitromethanide (1e) and $2 \mathrm{c}$ at $630 \mathrm{~nm}$ at $20{ }^{\circ} \mathrm{C}$ in water (J\&M).

\begin{tabular}{|c|c|c|c|c|c|}
\hline No. & $\begin{array}{c}{[\mathbf{2 c}]_{0} /} \\
\mathrm{mol} \mathrm{L}^{-1}\end{array}$ & $\begin{array}{l}{\left[\mathrm{OH}^{-}\right]_{0} /} \\
\mathrm{mol} \mathrm{L}^{-1}\end{array}$ & $\begin{array}{c}{[\mathbf{1 e - H}]_{0} /} \\
\mathrm{mol} \mathrm{L}^{-1}\end{array}$ & $\begin{array}{c}{[\mathbf{1 e}] /} \\
\mathrm{mol} \mathrm{L}^{-1}\end{array}$ & $\begin{array}{l}{\left[\mathrm{OH}^{-}\right] /} \\
\mathrm{mol} \mathrm{L}^{-1}\end{array}$ \\
\hline BUT-229-4 & $1.49 \times 10^{-5}$ & $1.16 \times 10^{-4}$ & $1.39 \times 10^{-4}$ & $1.15 \times 10^{-4}$ & $6.29 \times 10^{-7}$ \\
\hline BUT-229-1 & $1.69 \times 10^{-5}$ & $2.63 \times 10^{-4}$ & $2.96 \times 10^{-4}$ & $2.62 \times 10^{-4}$ & $9.93 \times 10^{-7}$ \\
\hline BUT-229-2 & $1.73 \times 10^{-5}$ & $5.38 \times 10^{-4}$ & $6.05 \times 10^{-4}$ & $5.37 \times 10^{-4}$ & $1.02 \times 10^{-6}$ \\
\hline BUT-229-3 & $1.56 \times 10^{-5}$ & $9.66 \times 10^{-4}$ & $1.09 \times 10^{-3}$ & $9.65 \times 10^{-4}$ & $9.95 \times 10^{-7}$ \\
\hline No. & $\begin{array}{c}k_{1 \Psi, \mathrm{obs}} / \\
\mathrm{s}^{-1}\end{array}$ & $\begin{array}{c}\text { Conv. / } \\
\% \\
\end{array}$ & $\begin{array}{c}k_{1 \Psi, \mathrm{OH}^{-}} / \\
\mathrm{s}^{-1, a} \\
\end{array}$ & $\begin{array}{c}k_{1 \Psi} / \\
\mathrm{s}^{-1} \\
\end{array}$ & \\
\hline BUT-229-4 & $2.08 \times 10^{-3}$ & 89 & $1.36 \times 10^{-6}$ & $2.08 \times 10^{-3}$ & \\
\hline BUT-229-1 & $6.36 \times 10^{-3}$ & 78 & $2.14 \times 10^{-6}$ & $6.36 \times 10^{-3}$ & \\
\hline BUT-229-2 & $1.83 \times 10^{-2}$ & 63 & $2.20 \times 10^{-6}$ & $1.83 \times 10^{-2}$ & \\
\hline BUT-229-3 & $3.08 \times 10^{-2}$ & 56 & $2.15 \times 10^{-6}$ & $3.08 \times 10^{-2}$ & \\
\hline
\end{tabular}

${ }^{a}$ Calculated with $k_{2, \mathrm{OH}^{-}}=2.16 \mathrm{~L} \mathrm{~mol}^{-1} \mathrm{~s}^{-1}$ from ref 11 .

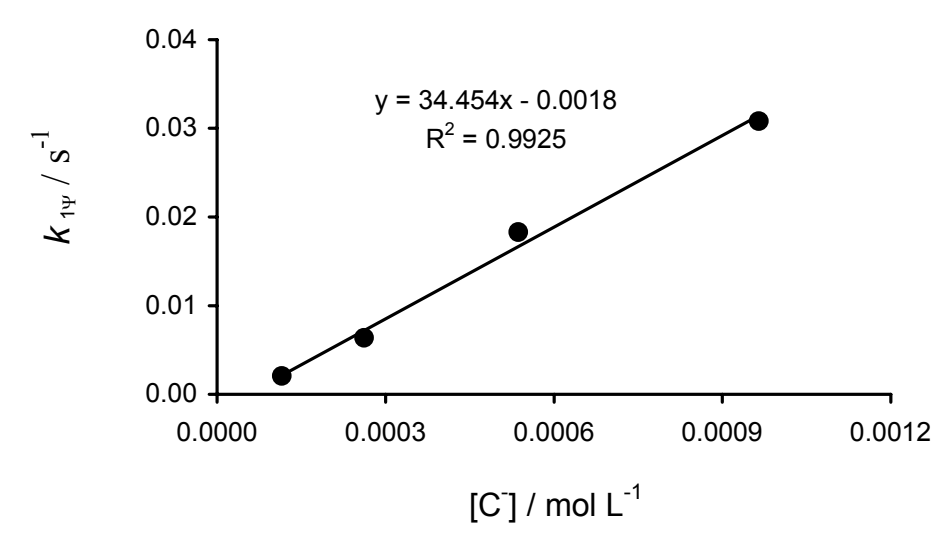

$k_{2, \mathrm{C}^{-}}=3.45 \times 10^{1} \mathrm{~L} \mathrm{~mol}^{-1} \mathrm{~s}^{-1}$ 
Table S18. Potassium salt of 4-nitrophenylnitromethanide (1f), 2a, and benzenesulfonic acid (BSA) at $605 \mathrm{~nm}$ at $20^{\circ} \mathrm{C}$ in water (Stopped flow).

\begin{tabular}{|c|c|c|c|c|c|}
\hline No. & $\begin{array}{c}{[\mathbf{2} \mathbf{a}]_{0} /} \\
\mathrm{mol} \mathrm{L}^{-1}\end{array}$ & $\begin{array}{l}{[\mathrm{BSA}] /} \\
\mathrm{mol} \mathrm{L}^{-1}\end{array}$ & $\begin{array}{l}{\left[\mathrm{OH}^{-}\right]_{0} /} \\
\mathrm{mol} \mathrm{L}^{-1, a}\end{array}$ & $\begin{array}{c}{[\mathbf{1 f}-\mathbf{H}]_{0} /} \\
\mathrm{mol} \mathrm{L}^{-1}\end{array}$ & $\begin{array}{l}{\left[\mathrm{OH}^{-}\right] /} \\
\mathrm{mol} \mathrm{L}^{-1}\end{array}$ \\
\hline BUT-234-4 & $9.82 \times 10^{-6}$ & $2.09 \times 10^{-5}$ & $1.29 \times 10^{-4}$ & $1.65 \times 10^{-4}$ & $2.79 \times 10^{-8}$ \\
\hline BUT-234-1 & $9.82 \times 10^{-6}$ & $2.09 \times 10^{-5}$ & $2.79 \times 10^{-4}$ & $3.30 \times 10^{-4}$ & $4.25 \times 10^{-8}$ \\
\hline BUT-234-2 & $9.82 \times 10^{-6}$ & $2.09 \times 10^{-5}$ & $5.79 \times 10^{-4}$ & $6.61 \times 10^{-4}$ & $5.48 \times 10^{-8}$ \\
\hline BUT-234-3 & $9.82 \times 10^{-6}$ & $2.09 \times 10^{-5}$ & $1.18 \times 10^{-3}$ & $1.32 \times 10^{-3}$ & $6.49 \times 10^{-8}$ \\
\hline No. & $\begin{array}{c}{[\mathbf{1 f}] /} \\
\mathrm{mol} \mathrm{L}^{-1}\end{array}$ & $\begin{array}{c}k_{1 \Psi, \mathrm{obs}} / \\
\mathrm{s}^{-1}\end{array}$ & $\begin{array}{c}k_{1 \Psi, \mathrm{OH}^{-}} / \\
\mathrm{s}^{-1, b}\end{array}$ & $\begin{array}{c}k_{1 \Psi} / \\
\mathrm{s}^{-1}\end{array}$ & \\
\hline BUT-234-4 & $1.29 \times 10^{-4}$ & $2.92 \times 10^{-1}$ & $3.65 \times 10^{-6}$ & $2.92 \times 10^{-1}$ & \\
\hline BUT-234-1 & $2.79 \times 10^{-4}$ & $6.27 \times 10^{-1}$ & $5.57 \times 10^{-6}$ & $6.27 \times 10^{-1}$ & \\
\hline BUT-234-2 & $5.79 \times 10^{-4}$ & 1.45 & $7.18 \times 10^{-6}$ & 1.45 & \\
\hline BUT-234-3 & $1.18 \times 10^{-3}$ & 3.13 & $8.51 \times 10^{-6}$ & 3.13 & \\
\hline
\end{tabular}

${ }^{a}$ Remaining $\left[\mathrm{OH}^{-}\right]_{0}$ after neutralization by benzenesulfonic acid (BSA).

${ }^{b}$ Calculated with $k_{2, \mathrm{OH}^{-}}=1.31 \times 10^{2} \mathrm{~L} \mathrm{~mol}^{-1} \mathrm{~s}^{-1}$ from ref 11 .

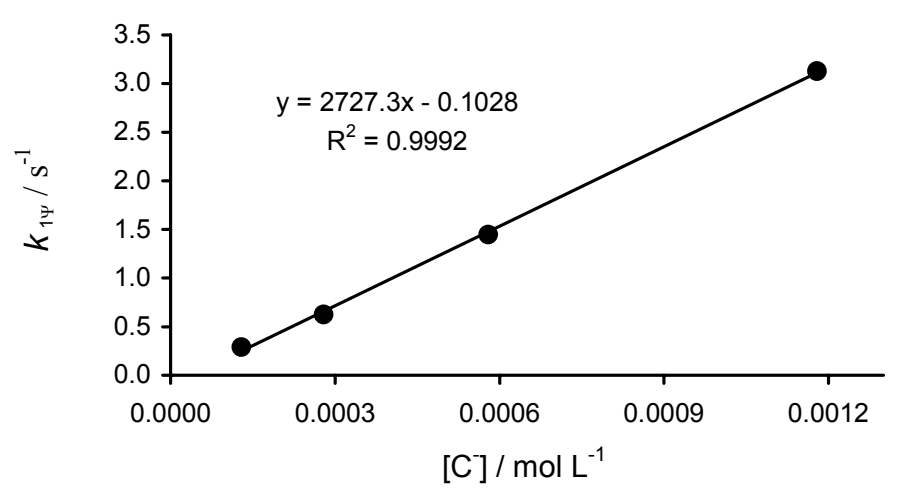

$k_{2, \mathrm{C}^{-}}=2.73 \times 10^{3} \mathrm{~L} \mathrm{~mol}^{-1} \mathrm{~s}^{-1}$ 
Table S19. Potassium salt of 4-nitrophenylnitromethanide (1f) and $\mathbf{2 b}$ at $620 \mathrm{~nm}$ at $20{ }^{\circ} \mathrm{C}$ in water $(\mathrm{J} \& \mathrm{M})$.

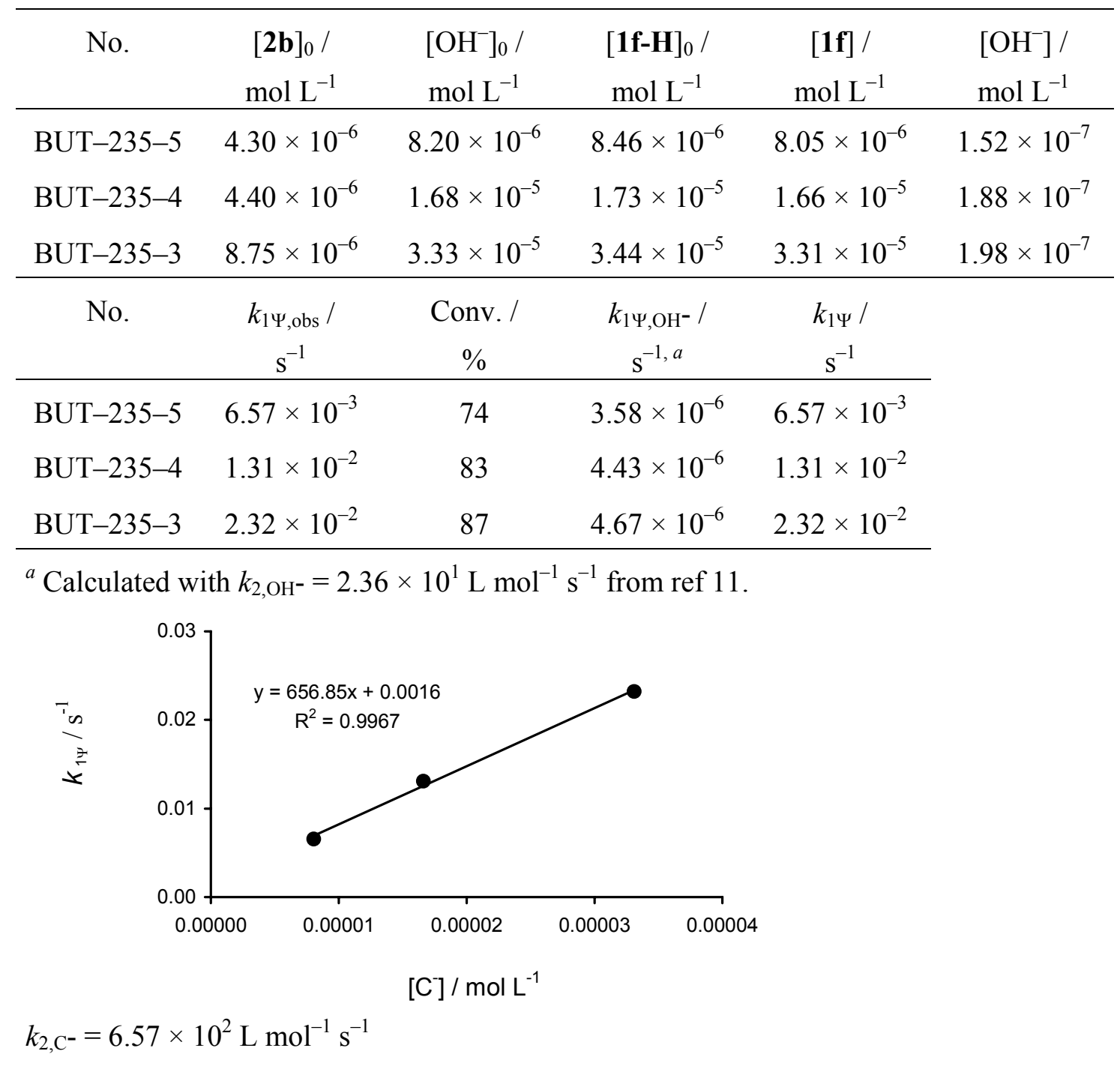


Table S20. Potassium salt of 4-nitrophenylnitromethanide (1f) and $\mathbf{2 c}$ at $630 \mathrm{~nm}$ at $20{ }^{\circ} \mathrm{C}$ in water (J\&M).

\begin{tabular}{|c|c|c|c|c|c|}
\hline No. & $\begin{array}{c}{[\mathbf{2 c}]_{0} /} \\
\mathrm{mol} \mathrm{L}^{-1}\end{array}$ & $\begin{array}{l}{\left[\mathrm{OH}^{-}\right]_{0} /} \\
\mathrm{mol} \mathrm{L}^{-1}\end{array}$ & $\begin{array}{c}{[\mathbf{1} \mathbf{f}-\mathbf{H}]_{0} /} \\
\mathrm{mol} \mathrm{L}^{-1}\end{array}$ & $\begin{array}{c}{[\mathbf{1 f}] /} \\
\mathrm{mol} \mathrm{L}^{-1}\end{array}$ & $\begin{array}{l}{\left[\mathrm{OH}^{-}\right] /} \\
\mathrm{mol} \mathrm{L}^{-1}\end{array}$ \\
\hline BUT-236-4 & $7.85 \times 10^{-6}$ & $1.63 \times 10^{-5}$ & $1.68 \times 10^{-5}$ & $1.61 \times 10^{-5}$ & $1.83 \times 10^{-7}$ \\
\hline BUT-236-3 & $7.77 \times 10^{-6}$ & $4.02 \times 10^{-5}$ & $4.15 \times 10^{-5}$ & $4.00 \times 10^{-5}$ & $2.06 \times 10^{-7}$ \\
\hline BUT-236-1 & $7.92 \times 10^{-6}$ & $8.20 \times 10^{-5}$ & $8.46 \times 10^{-5}$ & $8.18 \times 10^{-5}$ & $2.25 \times 10^{-7}$ \\
\hline BUT-236-2 & $7.46 \times 10^{-6}$ & $1.54 \times 10^{-4}$ & $1.59 \times 10^{-4}$ & $1.54 \times 10^{-4}$ & $2.28 \times 10^{-7}$ \\
\hline No. & $\begin{array}{c}k_{1 \Psi, \mathrm{obs}} / \\
\mathrm{s}^{-1}\end{array}$ & $\begin{array}{c}\text { Conv. / } \\
\%\end{array}$ & $\begin{array}{c}k_{1 \Psi, \mathrm{OH}^{-}} / \\
\mathrm{s}^{-1, a}\end{array}$ & $\begin{array}{c}k_{1 \Psi} / \\
\mathrm{s}^{-1}\end{array}$ & \\
\hline BUT-236-4 & $1.24 \times 10^{-3}$ & 44 & $3.96 \times 10^{-7}$ & $1.24 \times 10^{-3}$ & \\
\hline BUT-236-3 & $2.80 \times 10^{-3}$ & 79 & $4.45 \times 10^{-7}$ & $2.80 \times 10^{-3}$ & \\
\hline BUT-236-1 & $6.28 \times 10^{-3}$ & 71 & $4.85 \times 10^{-7}$ & $6.28 \times 10^{-3}$ & \\
\hline BUT-236-2 & $1.10 \times 10^{-2}$ & 90 & $4.93 \times 10^{-7}$ & $1.10 \times 10^{-2}$ & \\
\hline
\end{tabular}

${ }^{a}$ Calculated with $k_{2, \mathrm{OH}^{-}}=2.16 \mathrm{~L} \mathrm{~mol}^{-1} \mathrm{~s}^{-1}$ from ref 11 .

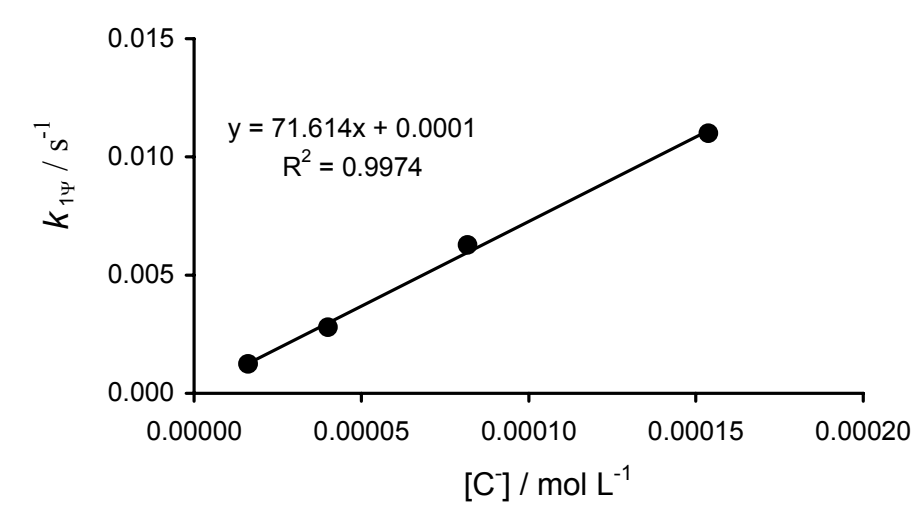

$k_{2, \mathrm{C}^{-}}=7.16 \times 10^{1} \mathrm{~L} \mathrm{~mol}^{-1} \mathrm{~s}^{-1}$ 
Table S21. Potassium salt of 3-nitrophenylnitromethanide (1g), 2a, and benzenesulfonic acid (BSA) at $605 \mathrm{~nm}$ at $20{ }^{\circ} \mathrm{C}$ in water (stopped-flow).

\begin{tabular}{|c|c|c|c|c|c|}
\hline No. & $\begin{array}{c}{[\mathbf{2} \mathbf{a}]_{0} /} \\
\mathrm{mol} \mathrm{L}^{-1}\end{array}$ & $\begin{array}{l}{[\mathrm{BSA}] /} \\
\mathrm{mol} \mathrm{L}^{-1}\end{array}$ & $\begin{array}{l}{\left[\mathrm{OH}^{-}\right]_{0} /} \\
\mathrm{mol} \mathrm{L}^{-1, a}\end{array}$ & $\begin{array}{c}{[\mathbf{1 g}-\mathbf{H}]_{0} /} \\
\mathrm{mol} \mathrm{L}^{-1}\end{array}$ & $\begin{array}{l}{\left[\mathrm{OH}^{-}\right] /} \\
\mathrm{mol} \mathrm{L}^{-1}\end{array}$ \\
\hline BUT-248-1 & $1.96 \times 10^{-5}$ & $2.98 \times 10^{-5}$ & $2.70 \times 10^{-4}$ & $3.23 \times 10^{-4}$ & $1.02 \times 10^{-7}$ \\
\hline BUT-248-2 & $1.96 \times 10^{-5}$ & $2.98 \times 10^{-5}$ & $5.70 \times 10^{-4}$ & $6.46 \times 10^{-4}$ & $1.50 \times 10^{-7}$ \\
\hline BUT-248-3 & $1.96 \times 10^{-5}$ & $2.98 \times 10^{-5}$ & $1.17 \times 10^{-3}$ & $1.29 \times 10^{-3}$ & $1.95 \times 10^{-7}$ \\
\hline BUT-248-4 & $1.96 \times 10^{-5}$ & $2.98 \times 10^{-5}$ & $1.77 \times 10^{-3}$ & $2.02 \times 10^{-3}$ & $1.41 \times 10^{-7}$ \\
\hline No. & $\begin{array}{c}{[\mathbf{1 g}] /} \\
\mathrm{mol} \mathrm{L}^{-1}\end{array}$ & $\begin{array}{c}k_{1 \Psi, \mathrm{obs}} / \\
\mathrm{s}^{-1}\end{array}$ & $\begin{array}{c}k_{1 \Psi, \mathrm{OH}^{-}} / \\
\mathrm{s}^{-1, b}\end{array}$ & $\begin{array}{c}k_{1 \Psi} / \\
\mathrm{s}^{-1}\end{array}$ & \\
\hline BUT-248-1 & $2.70 \times 10^{-4}$ & $5.82 \times 10^{-1}$ & $1.33 \times 10^{-5}$ & $5.82 \times 10^{-1}$ & \\
\hline BUT-248-2 & $5.70 \times 10^{-4}$ & 1.23 & $1.96 \times 10^{-5}$ & 1.23 & \\
\hline BUT-248-3 & $1.17 \times 10^{-3}$ & 2.43 & $2.55 \times 10^{-5}$ & 2.43 & \\
\hline BUT-248-4 & $1.77 \times 10^{-3}$ & 3.87 & $1.85 \times 10^{-5}$ & 3.87 & \\
\hline
\end{tabular}

${ }^{a}$ Remaining $\left[\mathrm{OH}^{-}\right]_{0}$ after neutralization by benzenesulfonic acid (BSA).

${ }^{b}$ Calculated with $k_{2, \mathrm{OH}^{-}}=1.31 \times 10^{2} \mathrm{~L} \mathrm{~mol}^{-1} \mathrm{~s}^{-1}$ from ref 11 .

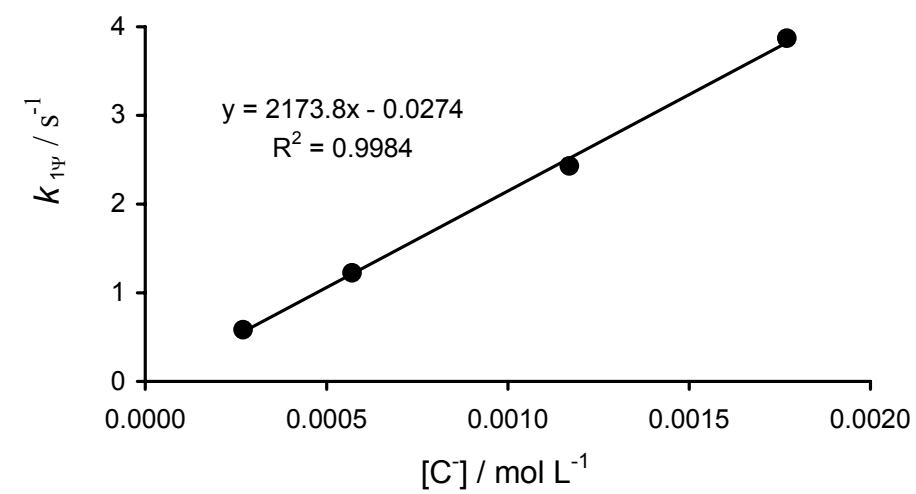

$k_{2, \mathrm{C}^{-}}=2.17 \times 10^{3} \mathrm{~L} \mathrm{~mol}^{-1} \mathrm{~s}^{-1}$ 
Table S22. Potassium salt of 3-nitrophenylnitromethanide (1g) and $\mathbf{2 b}$ at $620 \mathrm{~nm}$ at $20{ }^{\circ} \mathrm{C}$ in water (J\&M).

\begin{tabular}{|c|c|c|c|c|c|}
\hline No. & $\begin{array}{c}{[\mathbf{2} \mathbf{b}]_{0} /} \\
\mathrm{mol} \mathrm{L}^{-1}\end{array}$ & $\begin{array}{l}{\left[\mathrm{OH}^{-}\right]_{0} /} \\
\mathrm{mol} \mathrm{L}^{-1}\end{array}$ & $\begin{array}{c}{[\mathbf{1 g}-\mathbf{H}]_{0} /} \\
\mathrm{mol} \mathrm{L}^{-1}\end{array}$ & $\begin{array}{c}{[\mathbf{1 g}] /} \\
\mathrm{mol} \mathrm{L}^{-1}\end{array}$ & $\begin{array}{l}{\left[\mathrm{OH}^{-}\right] /} \\
\mathrm{mol} \mathrm{L}^{-1}\end{array}$ \\
\hline BUT-247-1 & $1.26 \times 10^{-5}$ & $3.00 \times 10^{-4}$ & $3.23 \times 10^{-4}$ & $3.00 \times 10^{-4}$ & $2.57 \times 10^{-7}$ \\
\hline BUT-247-2 & $1.26 \times 10^{-5}$ & $6.00 \times 10^{-4}$ & $6.46 \times 10^{-4}$ & $6.00 \times 10^{-4}$ & $2.59 \times 10^{-7}$ \\
\hline BUT-247-3 & $1.26 \times 10^{-5}$ & $1.20 \times 10^{-3}$ & $1.29 \times 10^{-3}$ & $1.20 \times 10^{-3}$ & $2.65 \times 10^{-7}$ \\
\hline BUT-247-4 & $1.26 \times 10^{-5}$ & $1.80 \times 10^{-3}$ & $2.02 \times 10^{-3}$ & $1.80 \times 10^{-3}$ & $1.63 \times 10^{-7}$ \\
\hline No. & $\begin{array}{c}k_{1 \Psi, \mathrm{obs}} / \\
\mathrm{s}^{-1}\end{array}$ & $\begin{array}{c}\text { Conv. / } \\
\%\end{array}$ & $\begin{array}{c}k_{1 \Psi, \mathrm{OH}^{-}} / \\
\mathrm{s}^{-1, a}\end{array}$ & $\begin{array}{c}k_{1 \Psi} / \\
\mathrm{s}^{-1}\end{array}$ & \\
\hline BUT-247-1 & $1.53 \times 10^{-1}$ & 74 & $6.07 \times 10^{-6}$ & $1.53 \times 10^{-1}$ & \\
\hline BUT-247-2 & $3.24 \times 10^{-1}$ & 83 & $6.10 \times 10^{-6}$ & $3.24 \times 10^{-1}$ & \\
\hline BUT-247-3 & $6.18 \times 10^{-1}$ & 87 & $6.26 \times 10^{-6}$ & $6.18 \times 10^{-1}$ & \\
\hline BUT-247-4 & 1.02 & 58 & $3.85 \times 10^{-6}$ & 1.02 & \\
\hline
\end{tabular}

${ }^{b}$ Calculated with $k_{2, \mathrm{OH}^{-}}=2.36 \times 10^{1} \mathrm{~L} \mathrm{~mol}^{-1} \mathrm{~s}^{-1}$ from ref 11 .

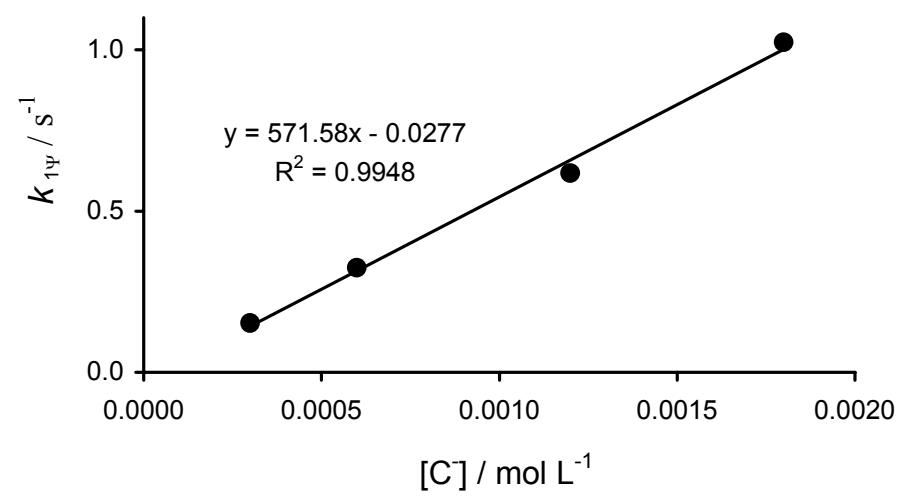

$k_{2, \mathrm{C}^{-}}=5.72 \times 10^{2} \mathrm{~L} \mathrm{~mol}^{-1} \mathrm{~s}^{-1}$ 
Table S23. Potassium salt of 3-nitrophenylnitromethanide (1g) and $\mathbf{2 c}$ at $605 \mathrm{~nm}$ at $20{ }^{\circ} \mathrm{C}$ in water (J\&M).

\begin{tabular}{ccccc}
\hline No. & $\begin{array}{c}{[\mathbf{2 c}]_{0} /} \\
\mathrm{mol} \mathrm{L}^{-1}\end{array}$ & $\begin{array}{c}{\left[\mathrm{OH}^{-}\right]_{0} /} \\
\mathrm{mol} \mathrm{L}^{-1}\end{array}$ & $\begin{array}{c}{[\mathbf{1 g - H}]_{0} /} \\
\mathrm{mol} \mathrm{L}^{-1}\end{array}$ & $\begin{array}{c}{[\mathbf{1 g}] /} \\
\mathrm{mol} \mathrm{L}^{-1}\end{array}$ \\
\hline BUT-246-1 & $1.78 \times 10^{-5}$ & $1.84 \times 10^{-4}$ & $1.86 \times 10^{-4}$ & $1.83 \times 10^{-4}$ \\
BUT-246-2 & $1.70 \times 10^{-5}$ & $3.51 \times 10^{-4}$ & $3.54 \times 10^{-4}$ & $3.49 \times 10^{-4}$ \\
BUT-246-3 & $1.70 \times 10^{-5}$ & $7.06 \times 10^{-4}$ & $7.12 \times 10^{-4}$ & $7.04 \times 10^{-4}$ \\
\hline No. & $k_{1 \Psi, \text { obs }} /$ & Conv. / & $k_{1 \Psi, \mathrm{OH}^{-}} /$ & $k_{1 \Psi} /$ \\
& $\mathrm{s}^{-1}$ & $\%$ & $\mathrm{~s}^{-1, a}$ & $\mathrm{~s}^{-1}$ \\
\hline BUT-246-1 & $2.28 \times 10^{-2}$ & 43 & $2.50 \times 10^{-6}$ & $2.28 \times 10^{-2}$ \\
BUT-246-2 & $3.45 \times 10^{-2}$ & 97 & $3.32 \times 10^{-6}$ & $3.45 \times 10^{-2}$ \\
BUT-246-3 & $6.79 \times 10^{-2}$ & 83 & $3.89 \times 10^{-6}$ & $6.79 \times 10^{-2}$ \\
\hline
\end{tabular}

${ }^{a}$ Calculated with $k_{2, \mathrm{OH}^{-}}=2.16 \mathrm{~L} \mathrm{~mol}^{-1} \mathrm{~s}^{-1}$ from ref 11 .

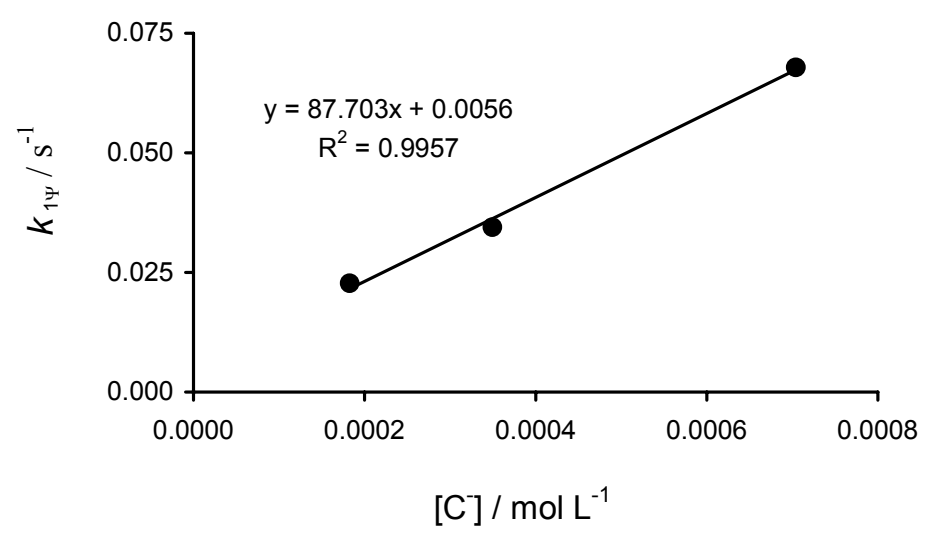

$k_{2, \mathrm{C}^{-}}=8.77 \times 10^{1} \mathrm{~L} \mathrm{~mol}^{-1} \mathrm{~s}^{-1}$ 
Table S24. Potassium salt of 4-cyanophenylnitromethanide (1h), 2a, and benzenesulfonic acid (BSA) at $600 \mathrm{~nm}$ at $20{ }^{\circ} \mathrm{C}$ in water (stopped-flow).

\begin{tabular}{|c|c|c|c|c|c|}
\hline No. & $\begin{array}{c}{[\mathbf{2 a}]_{0} /} \\
\mathrm{mol} \mathrm{L}^{-1}\end{array}$ & $\begin{array}{l}{[\mathrm{BSA}] /} \\
\mathrm{mol} \mathrm{L}^{-1}\end{array}$ & $\begin{array}{c}{\left[\mathrm{OH}^{-}\right]_{0} /} \\
\mathrm{mol} \mathrm{L}^{-1, a}\end{array}$ & $\begin{array}{c}{[\mathbf{1} \mathbf{h}-\mathbf{H}]_{0} /} \\
\mathrm{mol} \mathrm{L}^{-1}\end{array}$ & $\begin{array}{l}{\left[\mathrm{OH}^{-}\right] /} \\
\mathrm{mol} \mathrm{L}^{-1}\end{array}$ \\
\hline BUT-252-5 & $1.83 \times 10^{-5}$ & $2.32 \times 10^{-5}$ & $3.13 \times 10^{-5}$ & $5.61 \times 10^{-5}$ & $1.86 \times 10^{-8}$ \\
\hline BUT-252-4 & $1.83 \times 10^{-5}$ & $2.32 \times 10^{-5}$ & $8.58 \times 10^{-5}$ & $1.12 \times 10^{-4}$ & $4.83 \times 10^{-8}$ \\
\hline BUT-252-2 & $1.83 \times 10^{-5}$ & $2.32 \times 10^{-5}$ & $1.22 \times 10^{-4}$ & $1.46 \times 10^{-4}$ & $7.42 \times 10^{-8}$ \\
\hline BUT-252-3 & $1.83 \times 10^{-5}$ & $2.32 \times 10^{-5}$ & $2.50 \times 10^{-4}$ & $2.93 \times 10^{-4}$ & $8.53 \times 10^{-8}$ \\
\hline No. & $\begin{array}{c}{[\mathbf{1 h}] /} \\
\mathrm{mol} \mathrm{L}^{-1}\end{array}$ & $\begin{array}{c}k_{1 \Psi, \mathrm{obs}} / \\
\mathrm{s}^{-1}\end{array}$ & $\begin{array}{c}k_{1 \Psi, \mathrm{OH}^{-}} / \\
\mathrm{s}^{-1, b}\end{array}$ & $\begin{array}{c}k_{1 \Psi} / \\
\mathrm{s}^{-1}\end{array}$ & \\
\hline BUT-252-5 & $3.13 \times 10^{-5}$ & $1.54 \times 10^{-1}$ & $2.44 \times 10^{-6}$ & $1.54 \times 10^{-1}$ & \\
\hline BUT-252-4 & $8.58 \times 10^{-5}$ & $2.42 \times 10^{-1}$ & $6.33 \times 10^{-6}$ & $2.42 \times 10^{-1}$ & \\
\hline BUT-252-2 & $1.22 \times 10^{-4}$ & $2.80 \times 10^{-1}$ & $9.72 \times 10^{-6}$ & $2.80 \times 10^{-1}$ & \\
\hline BUT $-252-3$ & $2.50 \times 10^{-4}$ & $4.96 \times 10^{-1}$ & $1.12 \times 10^{-5}$ & $4.96 \times 10^{-1}$ & \\
\hline
\end{tabular}

${ }^{a}$ Remaining $\left[\mathrm{OH}^{-}\right]_{0}$ after neutralization by benzenesulfonic acid (BSA).

${ }^{b}$ Calculated with $k_{2, \mathrm{OH}^{-}}=1.31 \times 10^{2} \mathrm{~L} \mathrm{~mol}^{-1} \mathrm{~s}^{-1}$ from ref 11 .

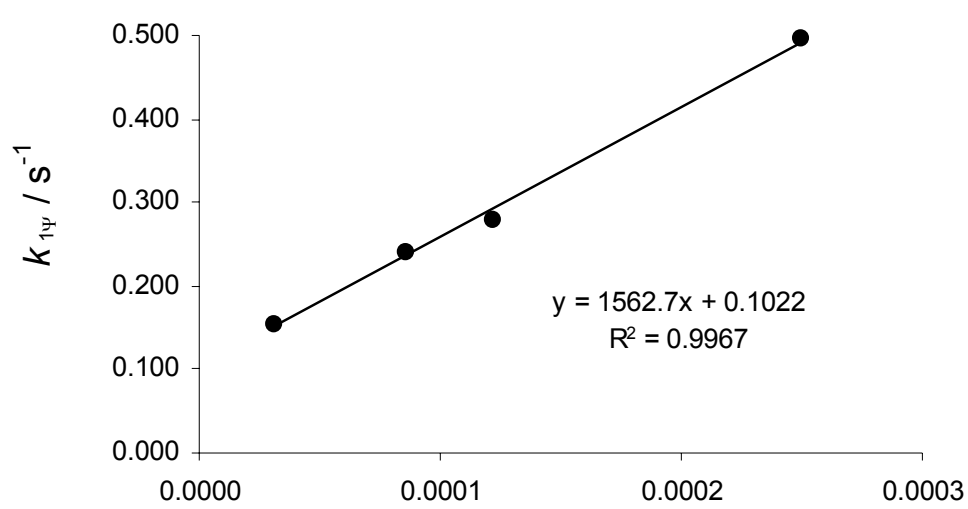

$\left[\mathrm{C}^{-}\right] / \mathrm{mol} \mathrm{L}^{-1}$

$k_{2, \mathrm{C}^{-}}=1.56 \times 10^{3} \mathrm{~L} \mathrm{~mol}^{-1} \mathrm{~s}^{-1}$ 
Table S25. Potassium salt of 4-cyanophenylnitromethanide (1h) and $\mathbf{2 b}$ at $620 \mathrm{~nm}$ at $20{ }^{\circ} \mathrm{C}$ in water (J\&M).

\begin{tabular}{|c|c|c|c|c|c|}
\hline No. & $\begin{array}{c}{[\mathbf{2} \mathbf{b}]_{0} /} \\
\mathrm{mol} \mathrm{L}^{-1}\end{array}$ & $\begin{array}{l}{\left[\mathrm{OH}^{-}\right]_{0} /} \\
\mathrm{mol} \mathrm{L}^{-1}\end{array}$ & $\begin{array}{c}{[\mathbf{1 h}-\mathbf{H}]_{0} /} \\
\mathrm{mol} \mathrm{L}^{-1}\end{array}$ & $\begin{array}{c}{[\mathbf{1 h}] /} \\
\mathrm{mol} \mathrm{L}^{-1}\end{array}$ & $\begin{array}{l}{\left[\mathrm{OH}^{-}\right] /} \\
\mathrm{mol} \mathrm{L}^{-1}\end{array}$ \\
\hline BUT-251-3 & $1.45 \times 10^{-5}$ & $1.07 \times 10^{-4}$ & $8.57 \times 10^{-5}$ & $8.56 \times 10^{-5}$ & $2.14 \times 10^{-5}$ \\
\hline BUT-251-2 & $1.53 \times 10^{-5}$ & $2.25 \times 10^{-4}$ & $1.81 \times 10^{-4}$ & $1.81 \times 10^{-4}$ & $4.41 \times 10^{-5}$ \\
\hline BUT-251-4 & $1.64 \times 10^{-5}$ & $4.03 \times 10^{-4}$ & $3.25 \times 10^{-4}$ & $3.25 \times 10^{-4}$ & $7.81 \times 10^{-5}$ \\
\hline BUT-251-6 & $1.65 \times 10^{-5}$ & $4.86 \times 10^{-4}$ & $4.89 \times 10^{-4}$ & $4.84 \times 10^{-4}$ & $1.57 \times 10^{-6}$ \\
\hline No. & $\begin{array}{c}k_{1 \Psi, \text { obs }} / \\
\mathrm{s}^{-1}\end{array}$ & $\begin{array}{c}\text { Conv. / } \\
\%\end{array}$ & $\begin{array}{c}k_{1 \Psi, \mathrm{OH}^{-}} / \\
\mathrm{s}^{-1, a}\end{array}$ & $\begin{array}{c}k_{1 \Psi} / \\
\mathrm{s}^{-1}\end{array}$ & \\
\hline BUT-251-3 & $3.62 \times 10^{-2}$ & 81 & $5.04 \times 10^{-4}$ & $3.57 \times 10^{-2}$ & \\
\hline BUT-251-2 & $9.05 \times 10^{-2}$ & 94 & $1.04 \times 10^{-3}$ & $8.95 \times 10^{-2}$ & \\
\hline BUT-251-4 & $1.67 \times 10^{-1}$ & 73 & $1.84 \times 10^{-3}$ & $1.65 \times 10^{-1}$ & \\
\hline BUT-251-6 & $2.28 \times 10^{-1}$ & 72 & $3.70 \times 10^{-5}$ & $2.28 \times 10^{-1}$ & \\
\hline
\end{tabular}

${ }^{a}$ Calculated with $k_{2, \mathrm{OH}^{-}}=2.36 \times 10^{1} \mathrm{~L} \mathrm{~mol}^{-1} \mathrm{~s}^{-1}$ from ref 11 .

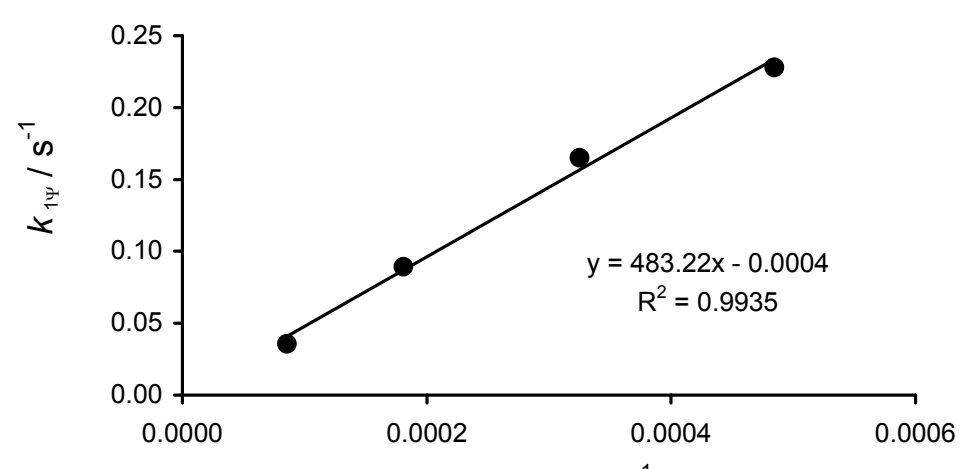

$\left[\mathrm{C}^{-}\right] / \mathrm{mol} \mathrm{L}^{-1}$

$k_{2, \mathrm{C}^{-}}=4.83 \times 10^{2} \mathrm{~L} \mathrm{~mol}^{-1} \mathrm{~s}^{-1}$ 
Table S26. Potassium salt of 4-cyanophenylnitromethanide (1h) and $\mathbf{2 c}$ at $630 \mathrm{~nm}$ at $20{ }^{\circ} \mathrm{C}$ in water (J\&M).

\begin{tabular}{|c|c|c|c|c|c|}
\hline No. & $\begin{array}{c}{[\mathbf{2} \mathbf{c}]_{0} /} \\
\mathrm{mol} \mathrm{L}^{-1}\end{array}$ & $\begin{array}{l}{\left[\mathrm{OH}^{-}\right]_{0} /} \\
\mathrm{mol} \mathrm{L}^{-1}\end{array}$ & $\begin{array}{c}{[\mathbf{1 h}-\mathbf{H}]_{0} /} \\
\mathrm{mol} \mathrm{L}^{-1}\end{array}$ & $\begin{array}{c}{[\mathbf{1 h}] /} \\
\mathrm{mol} \mathrm{L}^{-1}\end{array}$ & $\begin{array}{l}{\left[\mathrm{OH}^{-}\right] /} \\
\mathrm{mol} \mathrm{L}^{-1}\end{array}$ \\
\hline BUT-250-3 & $1.81 \times 10^{-5}$ & $1.75 \times 10^{-4}$ & $1.41 \times 10^{-4}$ & $1.41 \times 10^{-4}$ & $3.41 \times 10^{-5}$ \\
\hline BUT-250-2 & $1.67 \times 10^{-5}$ & $2.02 \times 10^{-4}$ & $2.49 \times 10^{-4}$ & $2.02 \times 10^{-4}$ & $6.35 \times 10^{-8}$ \\
\hline BUT-250-1 & $1.78 \times 10^{-5}$ & $6.48 \times 10^{-4}$ & $6.95 \times 10^{-4}$ & $6.48 \times 10^{-4}$ & $2.03 \times 10^{-7}$ \\
\hline BUT-250-4 & $1.77 \times 10^{-5}$ & $1.07 \times 10^{-3}$ & $1.03 \times 10^{-3}$ & $1.03 \times 10^{-3}$ & $4.04 \times 10^{-5}$ \\
\hline No. & $\begin{array}{c}k_{1 \Psi, \mathrm{obs}} / \\
\mathrm{s}^{-1}\end{array}$ & $\begin{array}{c}\text { Conv. / } \\
\%\end{array}$ & $\begin{array}{c}k_{1 \Psi, \mathrm{OH}^{-}} / \\
\mathrm{s}^{-1, a}\end{array}$ & $\begin{array}{c}k_{1 \Psi} / \\
\mathrm{s}^{-1}\end{array}$ & \\
\hline BUT-250-3 & $6.03 \times 10^{-3}$ & 65 & $7.36 \times 10^{-5}$ & $5.96 \times 10^{-3}$ & \\
\hline BUT-250-2 & $9.60 \times 10^{-3}$ & 77 & $1.37 \times 10^{-7}$ & $9.60 \times 10^{-3}$ & \\
\hline BUT-250-1 & $2.96 \times 10^{-2}$ & 87 & $4.38 \times 10^{-7}$ & $2.96 \times 10^{-2}$ & \\
\hline BUT-250-4 & $4.43 \times 10^{-2}$ & 79 & $8.72 \times 10^{-5}$ & $4.42 \times 10^{-2}$ & \\
\hline
\end{tabular}

${ }^{a}$ Calculated with $k_{2, \mathrm{OH}^{-}}=2.16 \mathrm{~L} \mathrm{~mol}^{-1} \mathrm{~s}^{-1}$ from ref 11 .

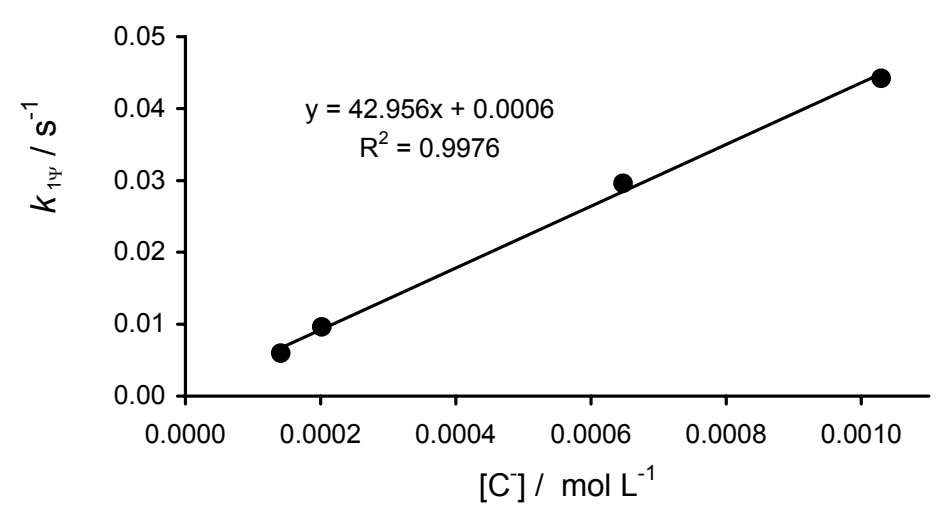

$k_{2, \mathrm{C}^{-}}=4.30 \times 10^{1} \mathrm{~L} \mathrm{~mol}^{-1} \mathrm{~s}^{-1}$ 


\subsection{Kinetic investigations of nitroalkyl anions in DMSO}

\subsubsection{Remarks}

- Nitroalkyl anions 1a-h were generated by deprotonation of the corresponding $\mathrm{CH}$ acids (CH) with the phosphazene bases $\mathrm{P}_{2}-t$-Bu (1-(tert-butylimino)-1,1,3,3,3pentakis(dimethylamino)- $1 \lambda^{5}, 3 \lambda^{5}$-diphosphazene; $\mathrm{p} K_{\mathrm{BH}+}=21.4$; Registry No. 111324-039), BEMP (2-tert-butylimino-1,3-dimethyl-2 $\lambda^{5}$-[1,3,2]-diazaphosphinan-2-yl)-diethylamine; $\mathrm{p} K_{\mathrm{BH}^{+}} \approx 16.5$; Registry No. 98015-45-3), and DBU (1,8-diazabicyclo[5.4.0]undec7-ene; $\mathrm{p} K_{\mathrm{BH}+} \approx 13.9$; Registry No. 6674-22-2). The counterions then were: $\mathrm{P}_{2}-t-\mathrm{Bu}-\mathrm{H}^{+}$, BEMP- $\mathrm{H}^{+}$, and DBU- $\mathrm{H}^{+}$. Benzhydrylium ions 2 were used as tetrafluoroborate salts.

- In the experiments with J\&M TIDAS $\left(\tau_{1 / 2}>10 \mathrm{~s}\right)$ three stock solutions have been used: the solution of the $\mathrm{CH}$ acid, the solution of the base, and the solution of the electrophile. In the first step, the carbanion was generated by addition of the base to the solution of the $\mathrm{CH}$ acid, then the electrophile was added and the data acquisition was started.

- For the stopped flow experiments $\left(\tau_{1 / 2}<10 \mathrm{~s}\right)$ two stock solutions were used: the solution of the electrophile and the solution of the carbanion generated from the $\mathrm{CH}$ acid and the base.

- As shown on the following pages, the plots of $k_{1 \Psi}$ increase with the concentration of the nucleophile, and the slopes of the linear correlations correspond to the second-order rateconstants $\left(k_{2}\right)$. 


\subsubsection{Individual kinetic runs in DMSO (Tables S27-S49)}

Table S27. $\left(\mathrm{P}_{2}-t-\mathrm{Bu}\right)-\mathrm{H}^{+}$salt of nitromethanide (1a) and $\mathbf{2 d}$ in DMSO at $\lambda=423 \mathrm{~nm}$ at $20.0{ }^{\circ} \mathrm{C}$ (stopped-flow).

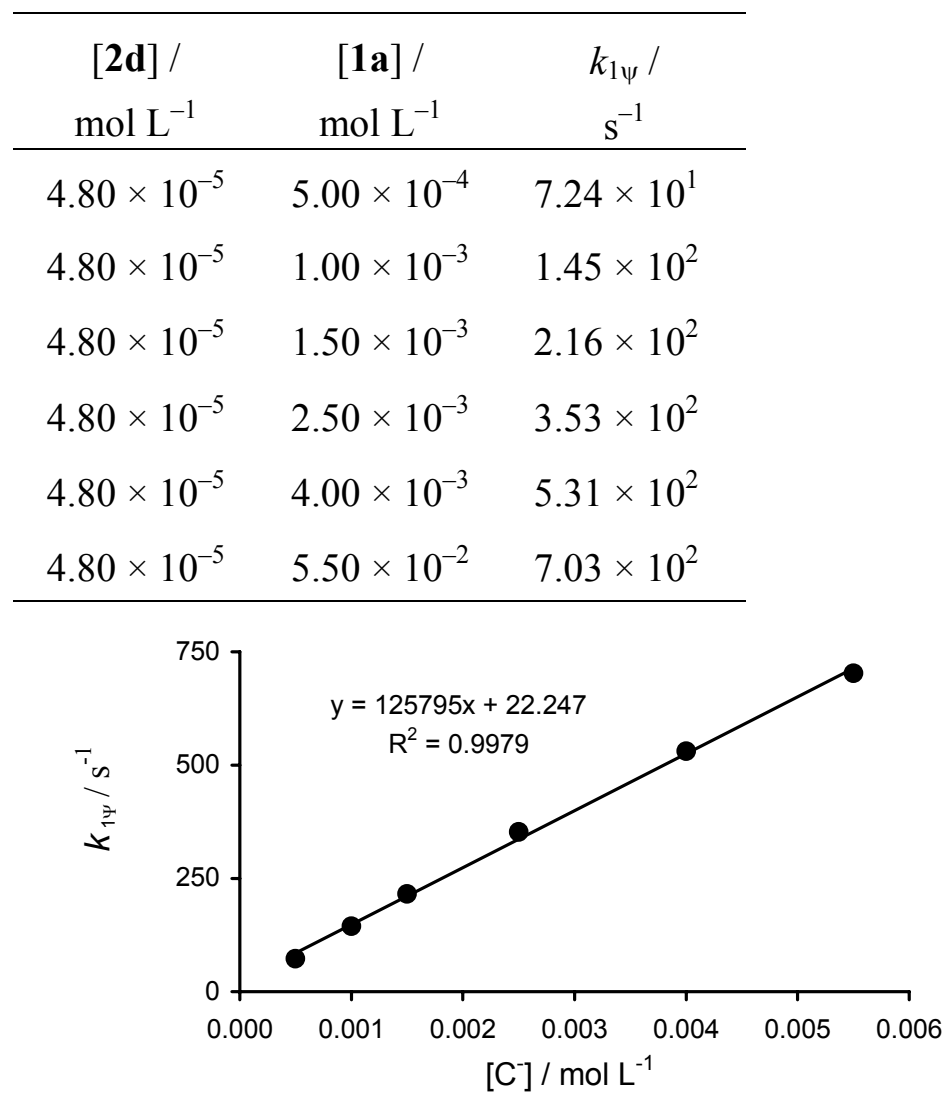

$k_{2, \mathrm{C}^{-}}=1.26 \times 10^{5} \mathrm{~L} \mathrm{~mol}^{-1} \mathrm{~s}^{-1}$ 
Table S28. $\left(\mathrm{P}_{2}-t-\mathrm{Bu}\right)-\mathrm{H}^{+}$salt of nitromethanide (1a) and $2 \mathrm{e}$ in DMSO at $\lambda=533 \mathrm{~nm}$ at $20.0{ }^{\circ} \mathrm{C}$ (stopped-flow).

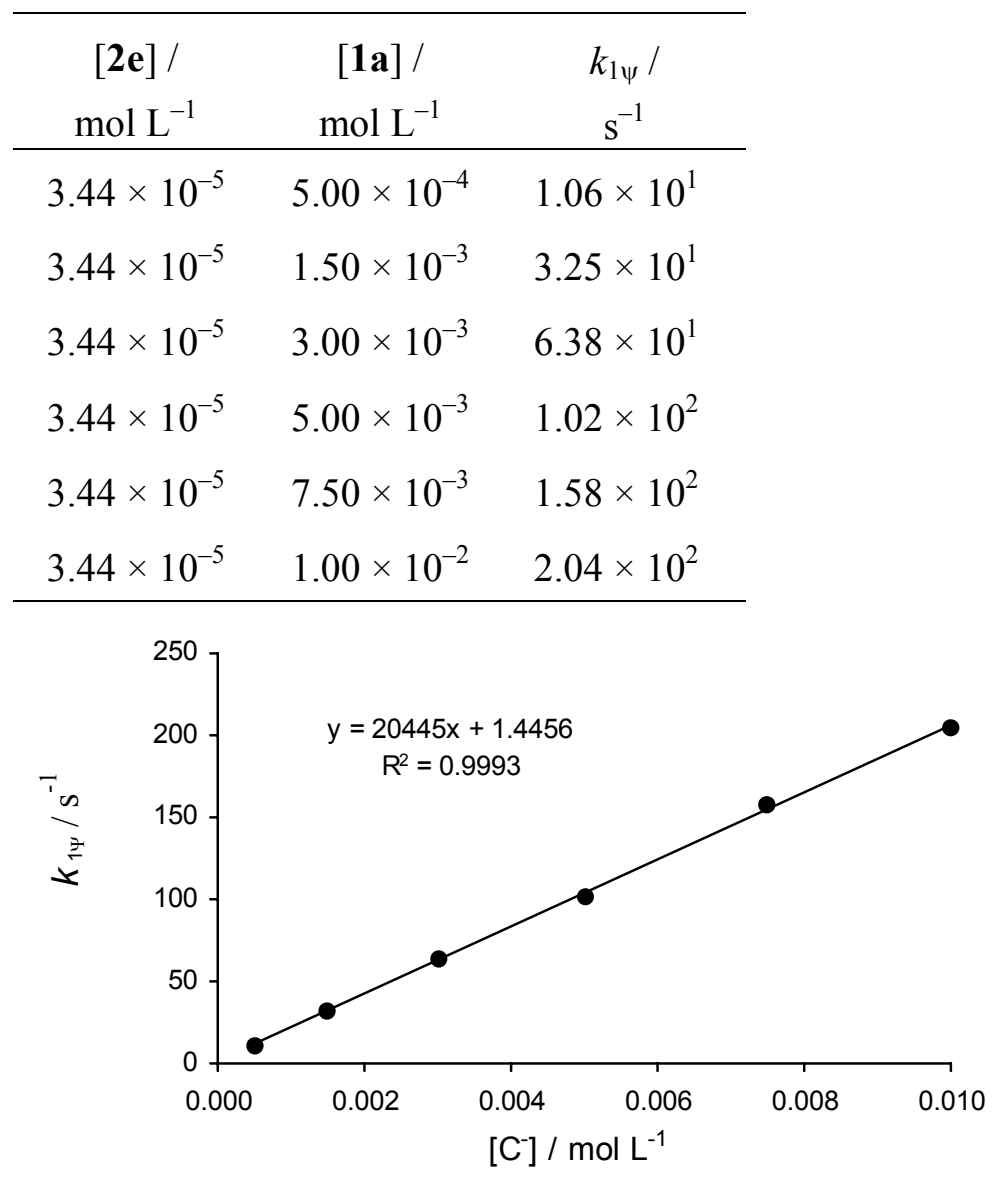

$k_{2, \mathrm{C}^{-}}=2.04 \times 10^{4} \mathrm{~L} \mathrm{~mol}^{-1} \mathrm{~s}^{-1}$ 
Table S29. $\left(\mathrm{P}_{2}-t-\mathrm{Bu}\right)-\mathrm{H}^{+}$salt of nitromethanide (1a) and $\mathbf{2 f}$ in DMSO at $\lambda=371 \mathrm{~nm}$ at $20.0{ }^{\circ} \mathrm{C}$ (stopped-flow).

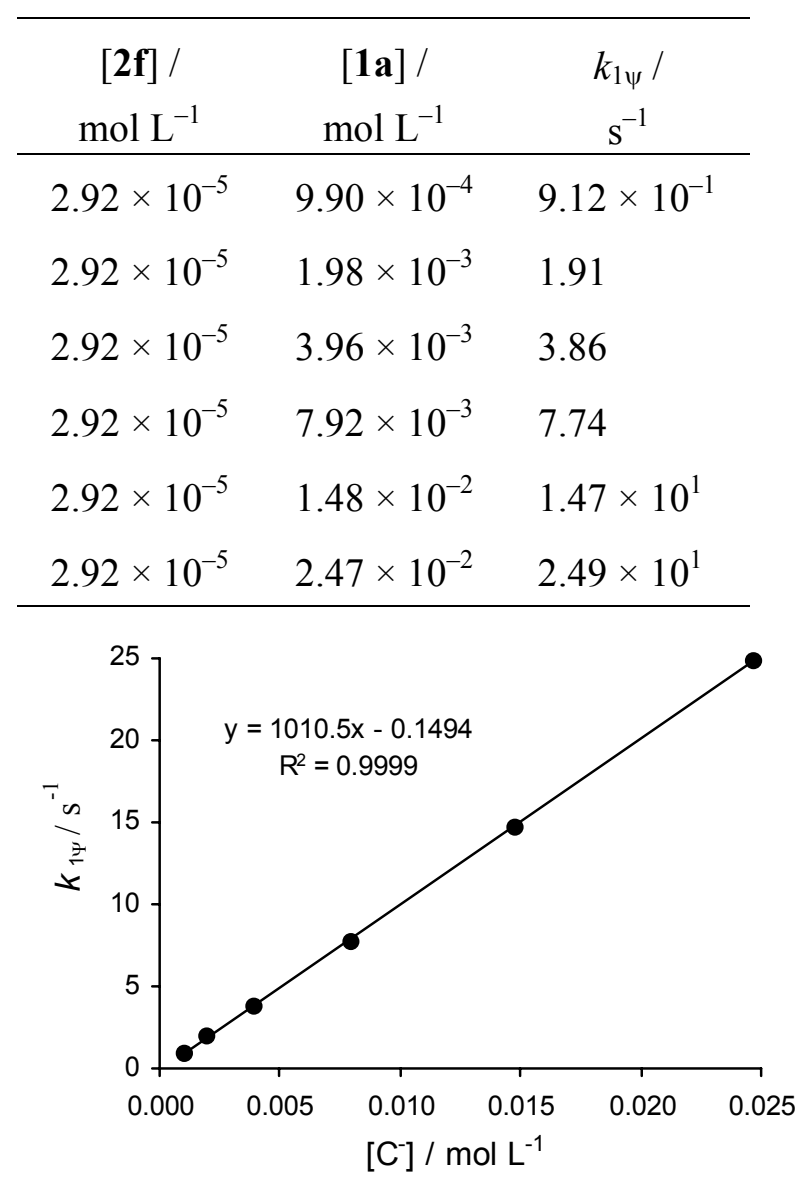

$k_{2, \mathrm{C}^{-}}=1.01 \times 10^{3} \mathrm{~L} \mathrm{~mol}^{-1} \mathrm{~s}^{-1}$ 
Table S30. $\left(\mathrm{P}_{2}-t-\mathrm{Bu}\right)-\mathrm{H}^{+}$salt of nitromethanide (1a) and $2 \mathbf{i}$ in DMSO at $\lambda=524 \mathrm{~nm}$ at $20.0{ }^{\circ} \mathrm{C}$ (J\&M).

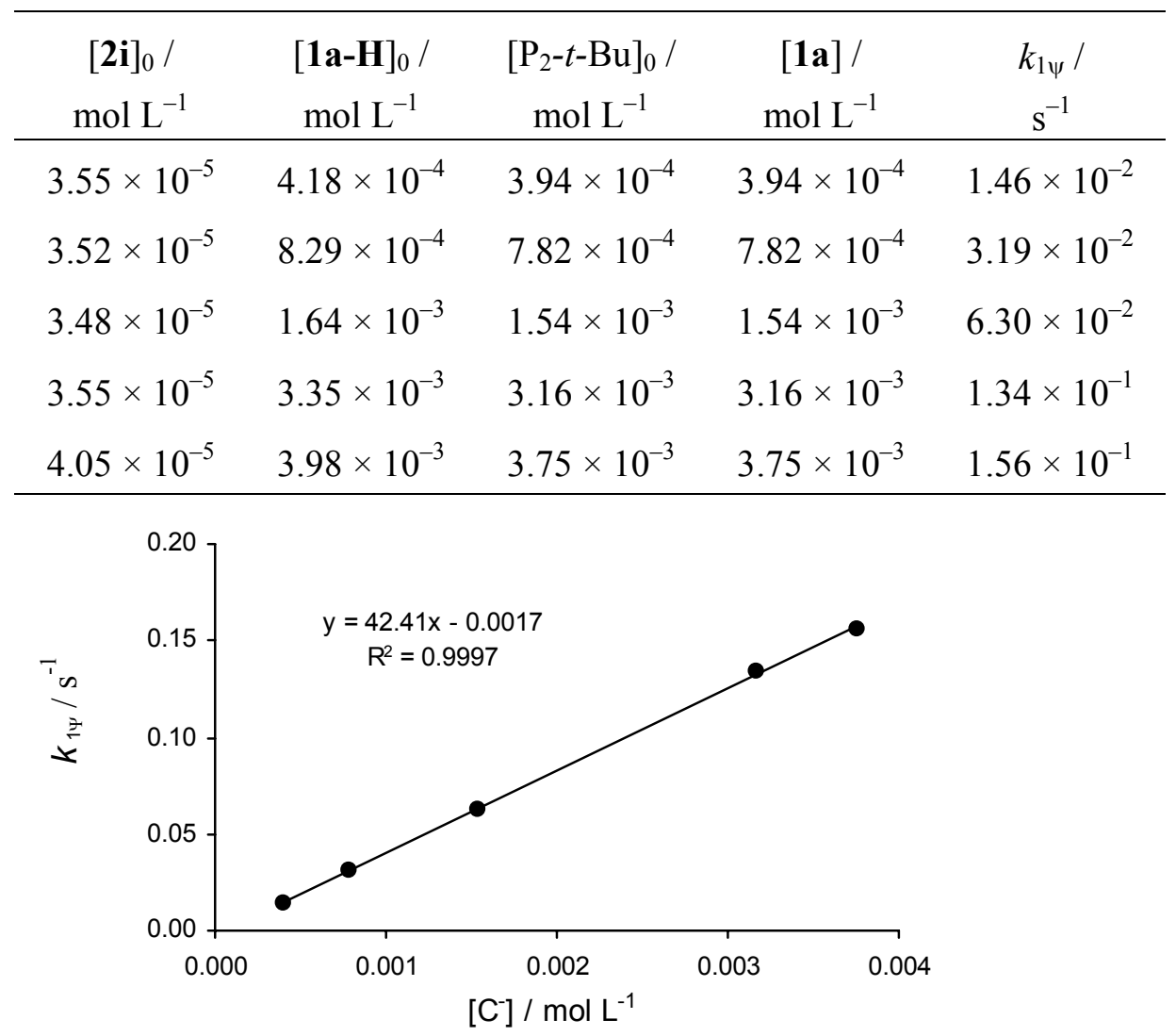

$k_{2, \mathrm{C}^{-}}=4.24 \times 10^{1} \mathrm{~L} \mathrm{~mol}^{-1} \mathrm{~s}^{-1}$ 
Table S31. $\left(\mathrm{P}_{2}-t-\mathrm{Bu}\right)-\mathrm{H}^{+}$salt of 2-nitropropanide (1c) and $2 \mathbf{d}$ in $\mathrm{DMSO}$ at $\lambda=420 \mathrm{~nm}$ at $20.0{ }^{\circ} \mathrm{C}$ (stopped-flow).

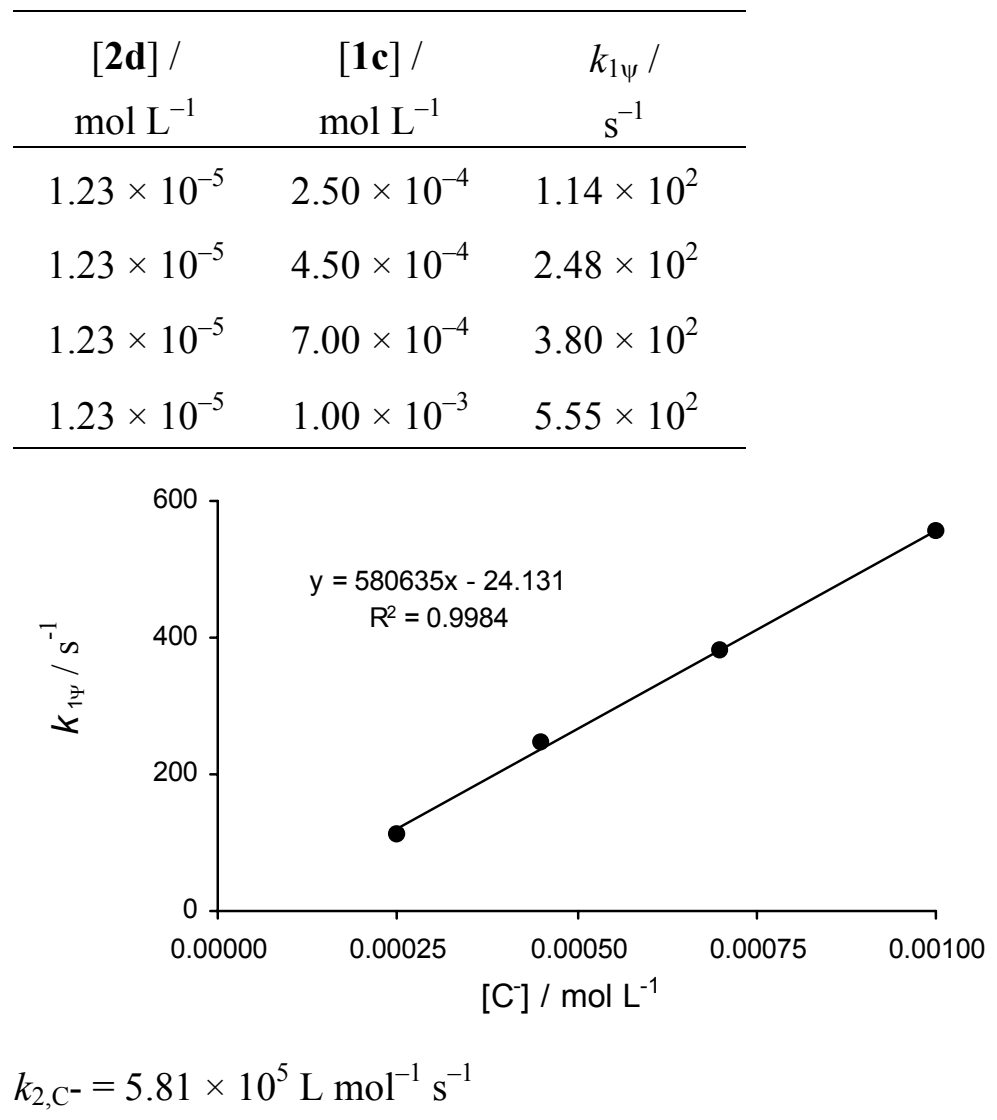


Table S32. $\left(\mathrm{P}_{2}-t-\mathrm{Bu}\right)-\mathrm{H}^{+}$salt of 2-nitropropanide (1c) and $2 \mathrm{e}$ in DMSO at $\lambda=533 \mathrm{~nm}$ at $20.0{ }^{\circ} \mathrm{C}$ (stopped-flow).

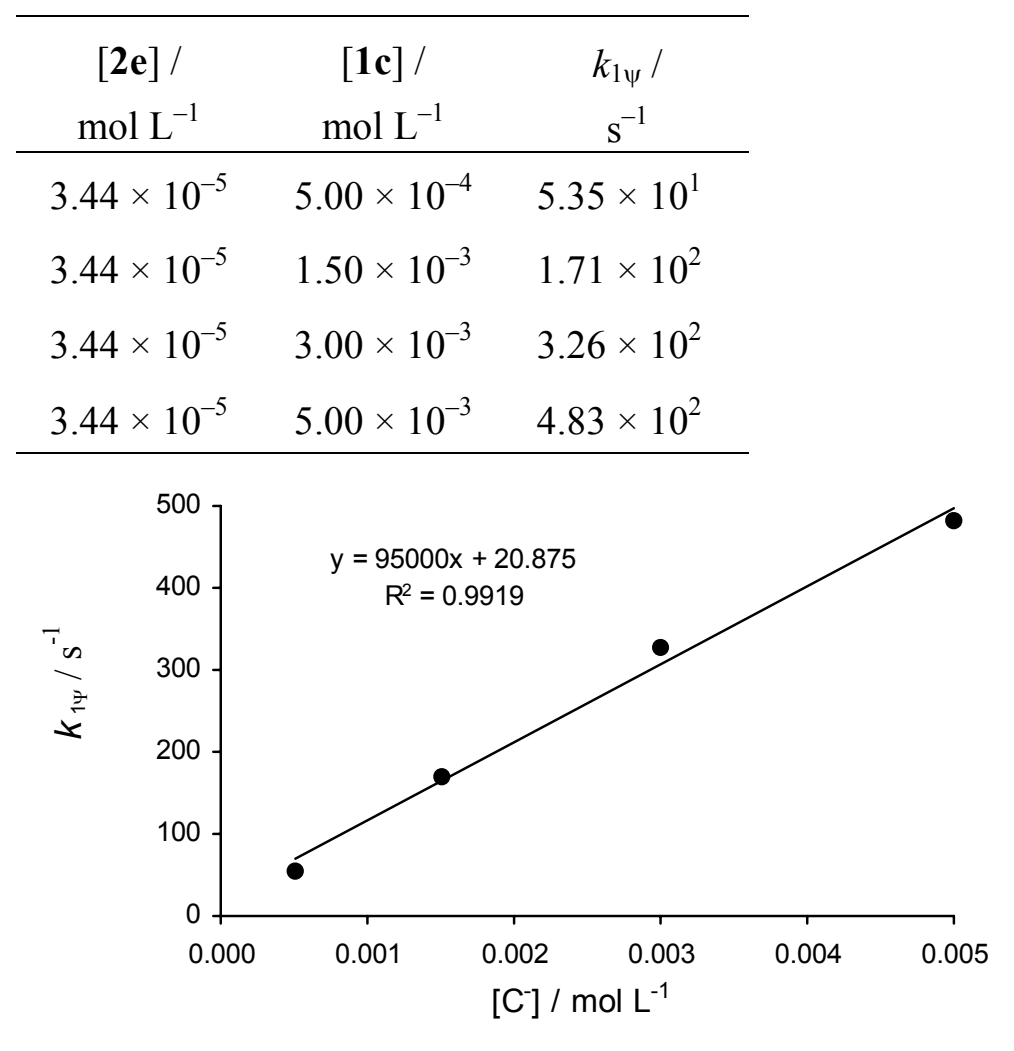

$k_{2, \mathrm{C}^{-}}=9.50 \times 10^{4} \mathrm{~L} \mathrm{~mol}^{-1} \mathrm{~s}^{-1}$ 
Table S33. $\left(\mathrm{P}_{2}-t-\mathrm{Bu}\right)-\mathrm{H}^{+}$salt of 2-nitropropanide (1c) and $\mathbf{2 f}$ in DMSO at $\lambda=371 \mathrm{~nm}$ at $20.0^{\circ} \mathrm{C}$ (stopped-flow).

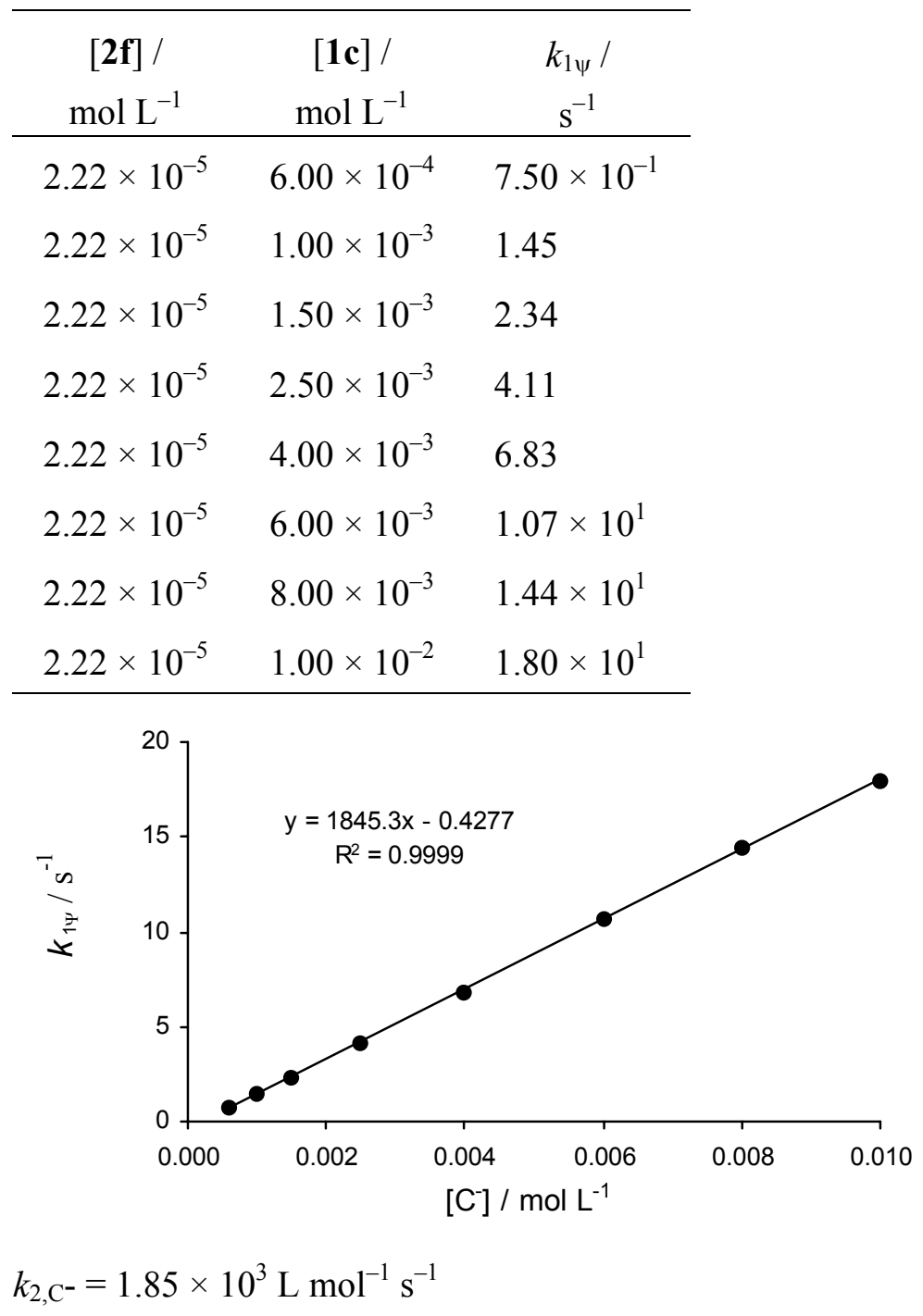


Table S34. $\left(\mathrm{P}_{2}-t-\mathrm{Bu}\right)-\mathrm{H}^{+}$salt of 2-nitropropanide (1c) and $2 \mathbf{i}$ in DMSO at $\lambda=524 \mathrm{~nm}$ at $20.0^{\circ} \mathrm{C}$ (stopped-flow).

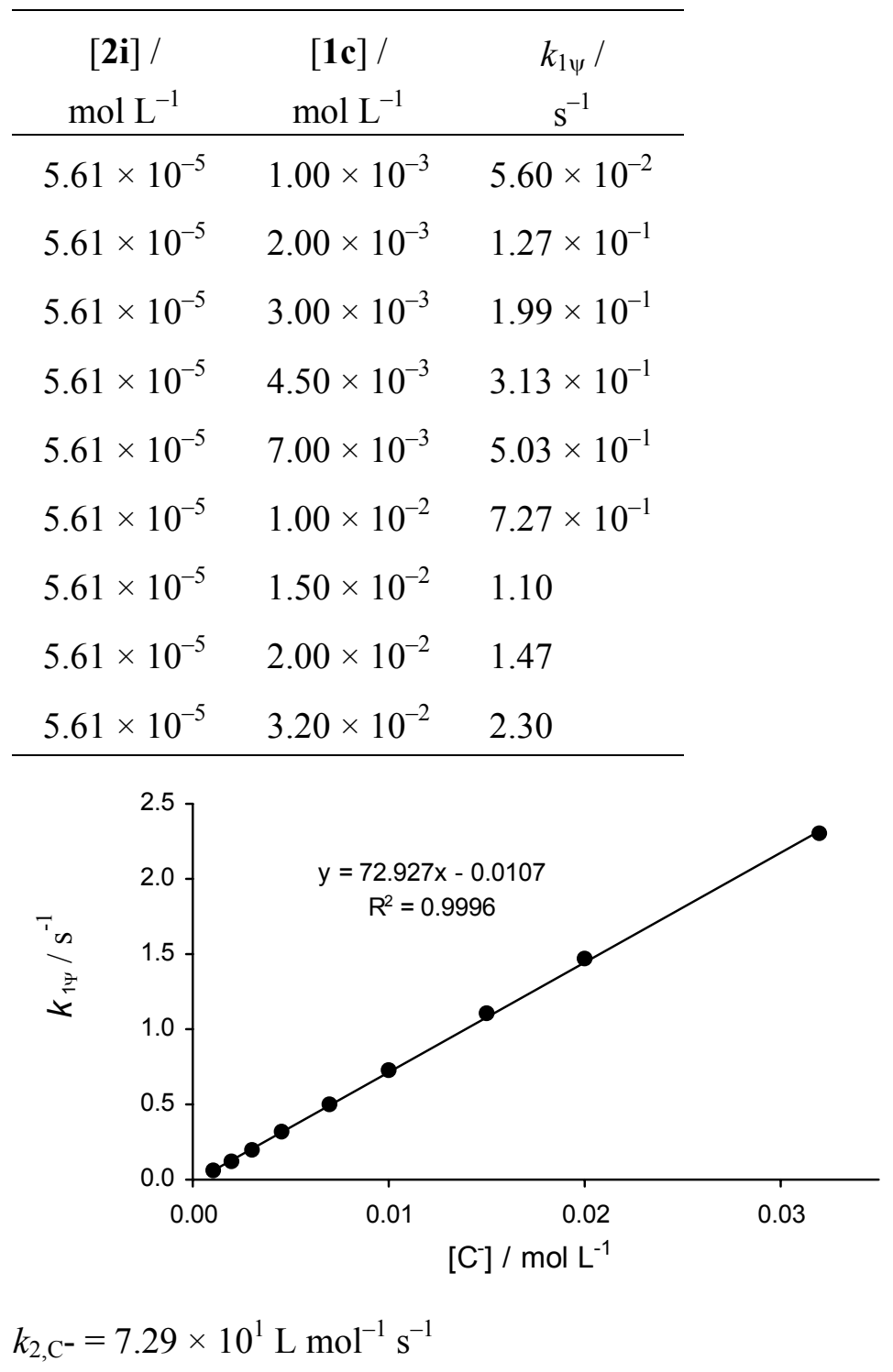


Table S35. (BEMP)- $\mathrm{H}^{+}$salt of phenylnitromethanide (1d) and $\mathbf{2 c}$ in DMSO at $\lambda=640 \mathrm{~nm}$ at 20.0 ${ }^{\circ} \mathrm{C}$ (stopped-flow).

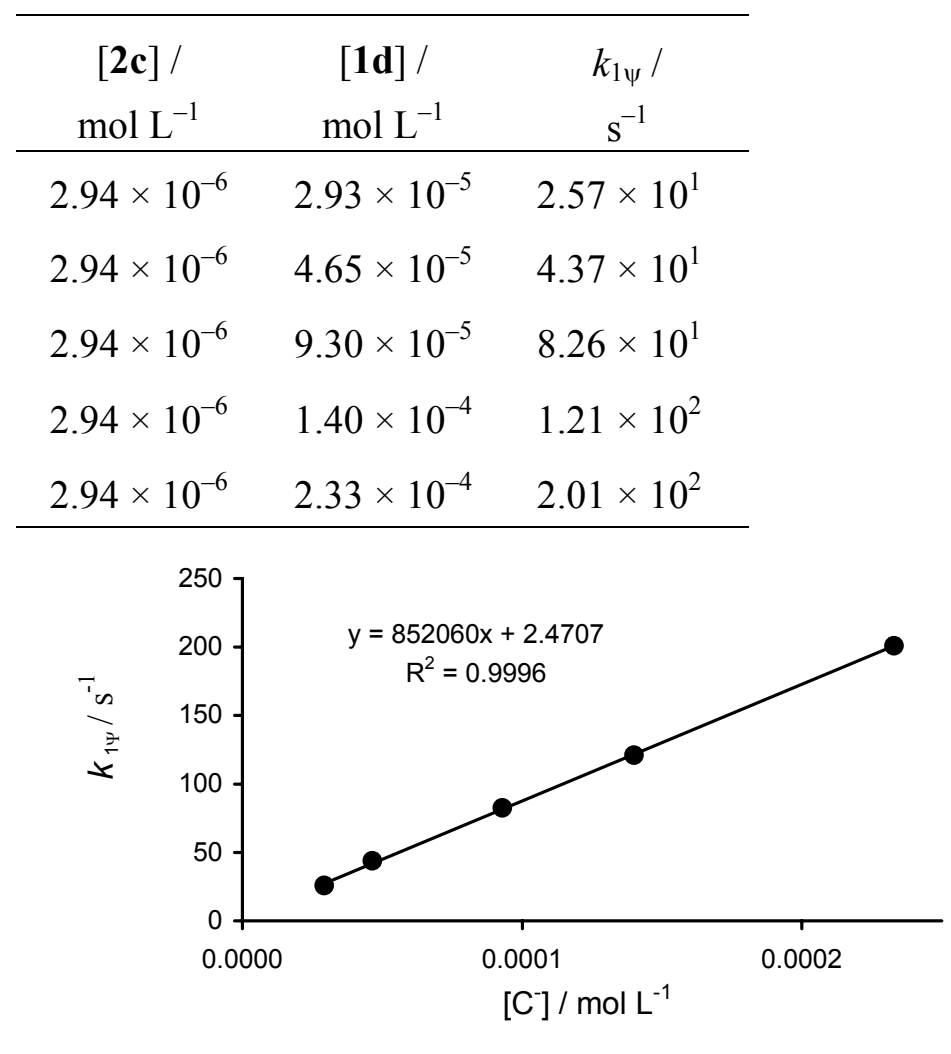

$k_{2, \mathrm{C}^{-}}=8.52 \times 10^{5} \mathrm{~L} \mathrm{~mol}^{-1} \mathrm{~s}^{-1}$ 
Table S36. (BEMP)- $\mathrm{H}^{+}$salt of phenylnitromethanide (1d) and $2 \mathbf{e}$ in DMSO at $\lambda=533 \mathrm{~nm}$ at 20.0 ${ }^{\circ} \mathrm{C}$ (stopped-flow).

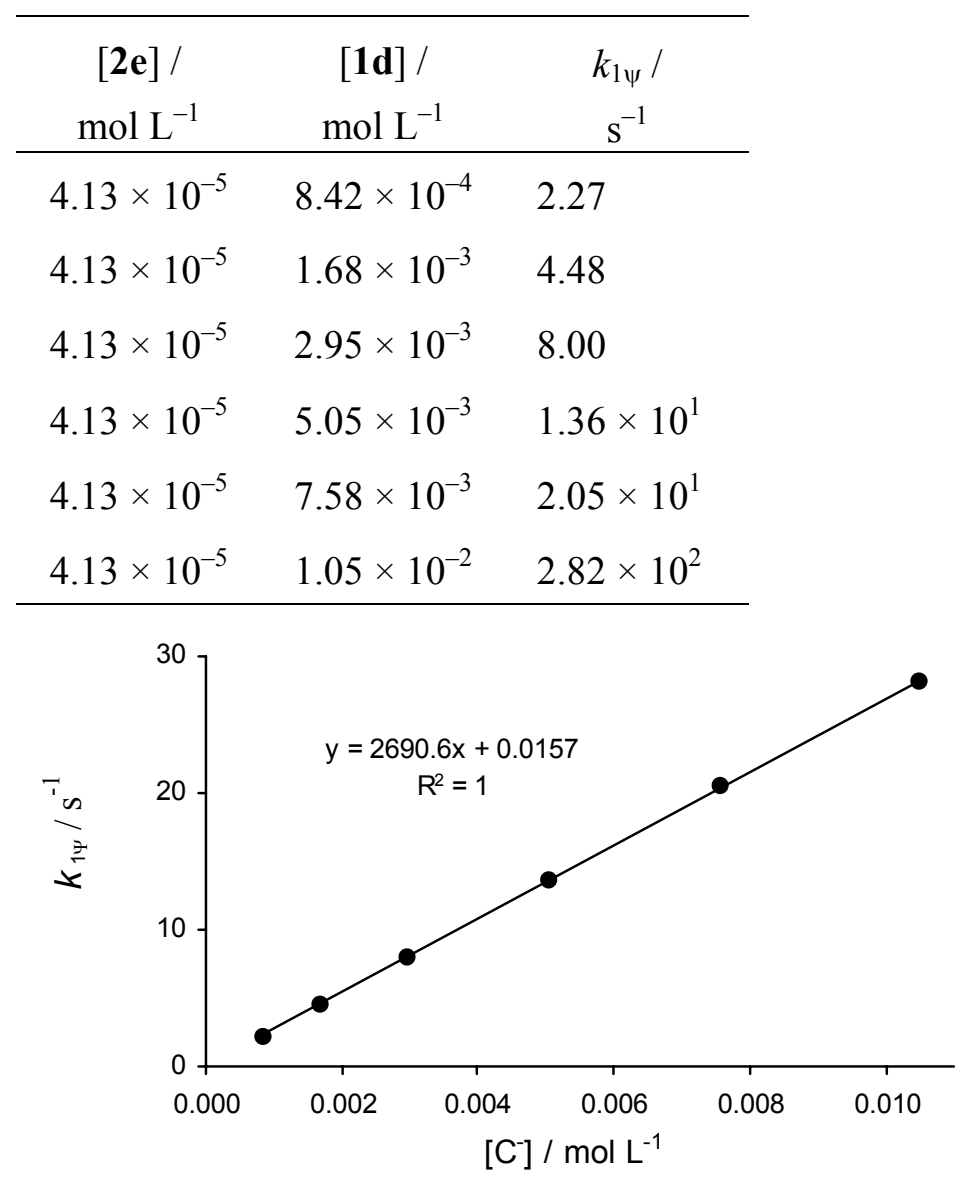

$k_{2, \mathrm{C}^{-}}=2.69 \times 10^{3} \mathrm{~L} \mathrm{~mol}^{-1} \mathrm{~s}^{-1}$ 
Table S37. (BEMP)- $\mathrm{H}^{+}$salt of phenylnitromethanide (1d) and $\mathbf{2 h}$ in DMSO at $\lambda=488 \mathrm{~nm}$ at 20.0 ${ }^{\circ} \mathrm{C}$ (J\&M).

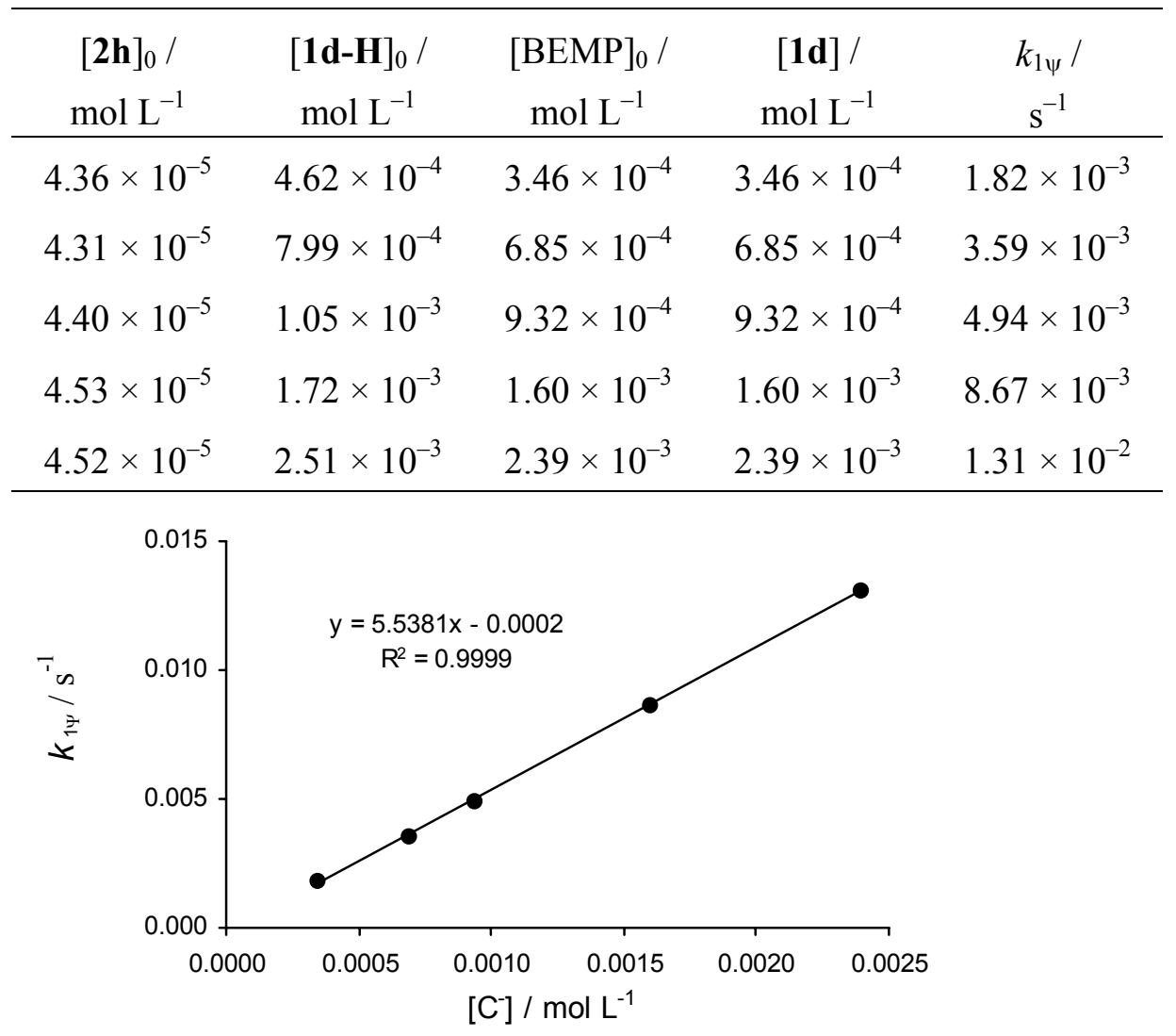

$k_{2, \mathrm{C}^{-}}=5.54 \mathrm{~L} \mathrm{~mol}^{-1} \mathrm{~s}^{-1}$ 
Table S38. (BEMP)- $\mathrm{H}^{+}$salt of 4-methylphenylnitromethanide (1e) and $2 \mathrm{c}$ in DMSO at $\lambda=640$ $\mathrm{nm}$ at $20.0{ }^{\circ} \mathrm{C}$ (stopped-flow).

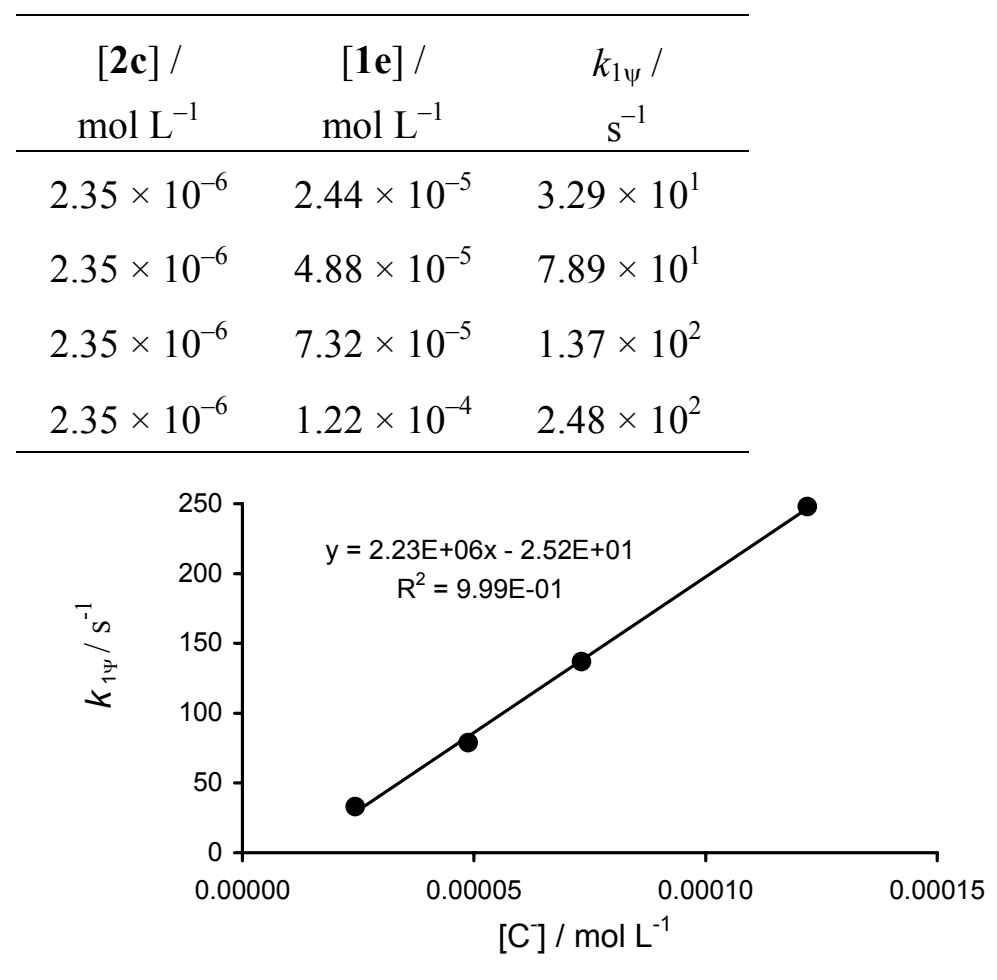

$k_{2, \mathrm{C}^{-}}=2.23 \times 10^{6} \mathrm{~L} \mathrm{~mol}^{-1} \mathrm{~s}^{-1}$ 
Table S39. (BEMP) $-\mathrm{H}^{+}$salt of 4-methylphenylnitromethanide (1e) and $2 \mathbf{e}$ in DMSO at $\lambda=533$ nm at $20.0{ }^{\circ} \mathrm{C}$ (stopped-flow).

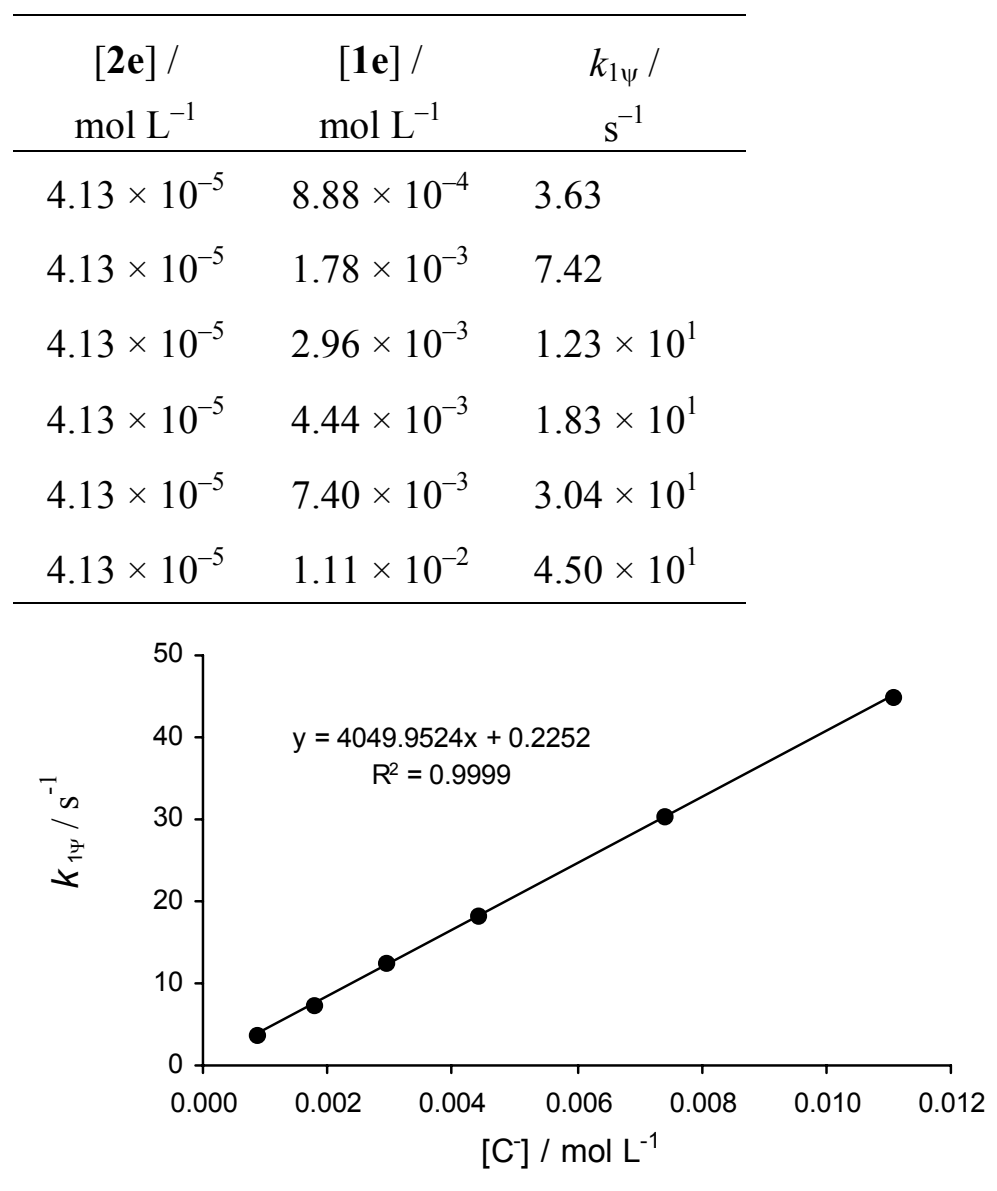

$k_{2, \mathrm{C}^{-}}=4.05 \times 10^{3} \mathrm{~L} \mathrm{~mol}^{-1} \mathrm{~s}^{-1}$ 
Table S40. (BEMP)- $\mathrm{H}^{+}$salt of 4-methylphenylnitromethanide (1e) and $\mathbf{2 h}$ in DMSO at $\lambda=488$ nm at $20.0{ }^{\circ} \mathrm{C}(\mathrm{J} \& \mathrm{M})$.

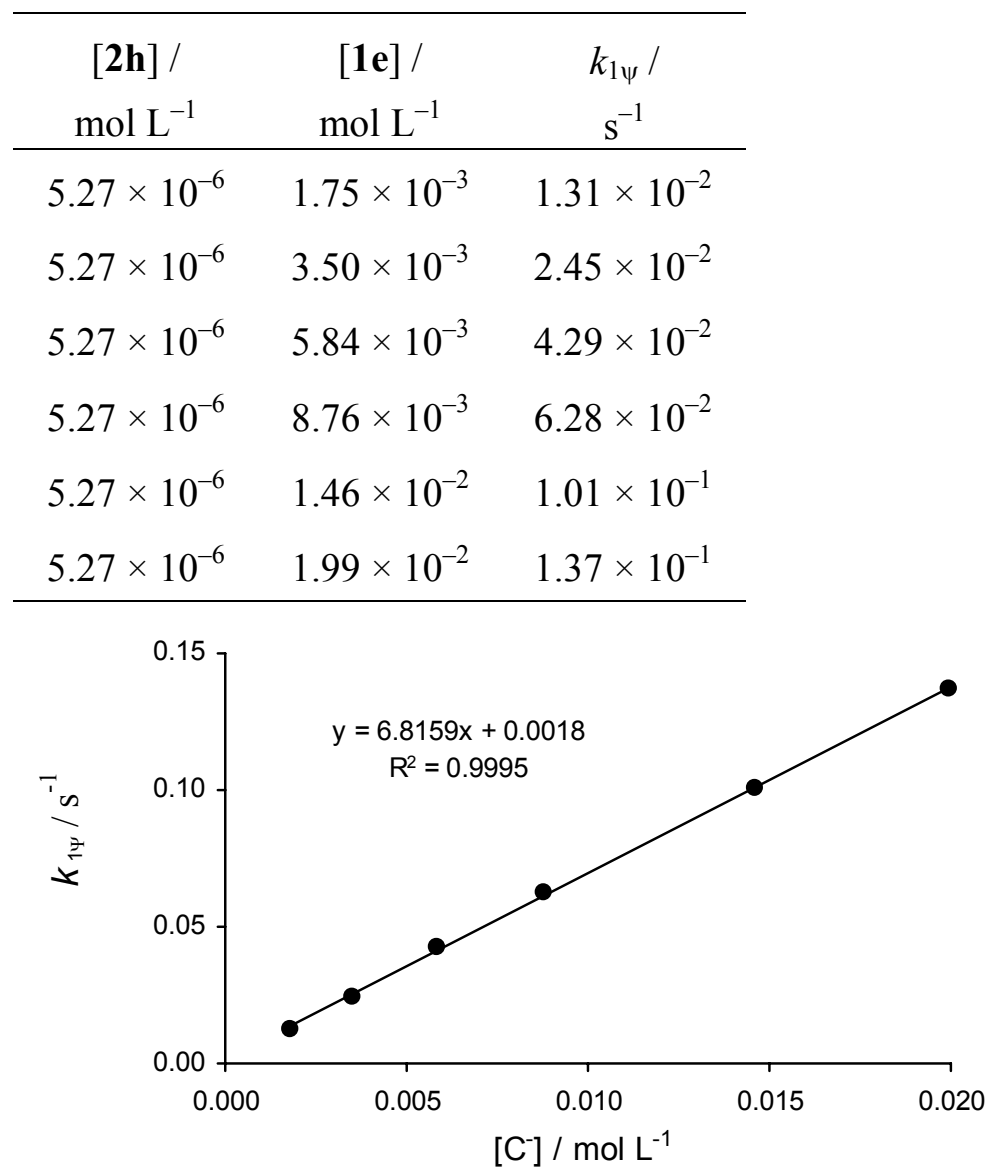

$k_{2, \mathrm{C}^{-}}=6.82 \mathrm{~L} \mathrm{~mol}^{-1} \mathrm{~s}^{-1}$ 
Table S41. (DBU)- $\mathrm{H}^{+}$salt of 4-nitrophenylnitromethanide (1f) and $\mathbf{2 b}$ in DMSO at $\lambda=630 \mathrm{~nm}$ at $20.0^{\circ} \mathrm{C}$ (stopped-flow).

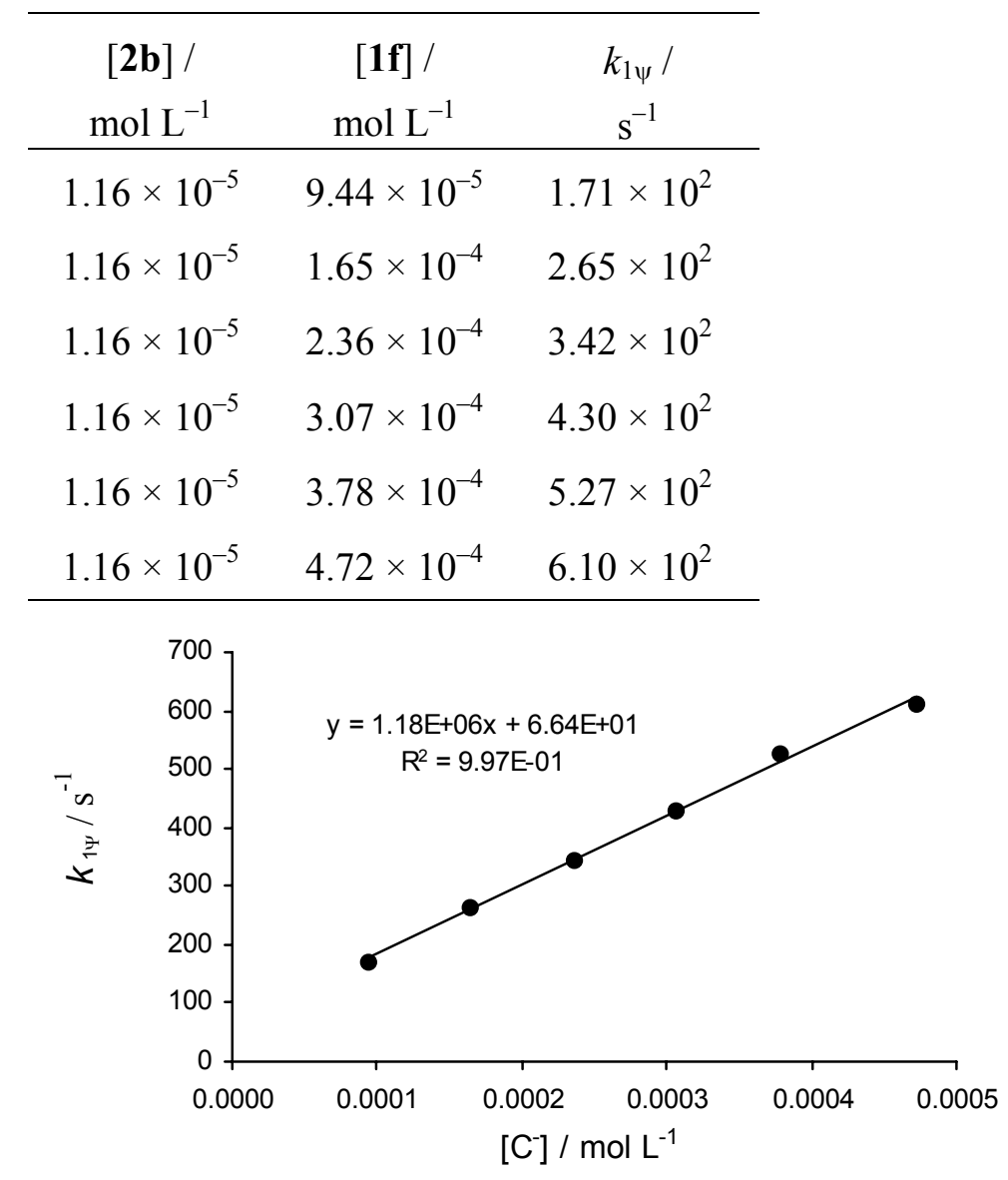

$k_{2, \mathrm{C}^{-}}=1.18 \times 10^{6} \mathrm{~L} \mathrm{~mol}^{-1} \mathrm{~s}^{-1}$ 
Table S42. (DBU)- $\mathrm{H}^{+}$salt of 4-nitrophenylnitromethanide (1f) and $2 \mathrm{c}$ in DMSO at $\lambda=640 \mathrm{~nm}$ at $20.0^{\circ} \mathrm{C}$ (stopped-flow).

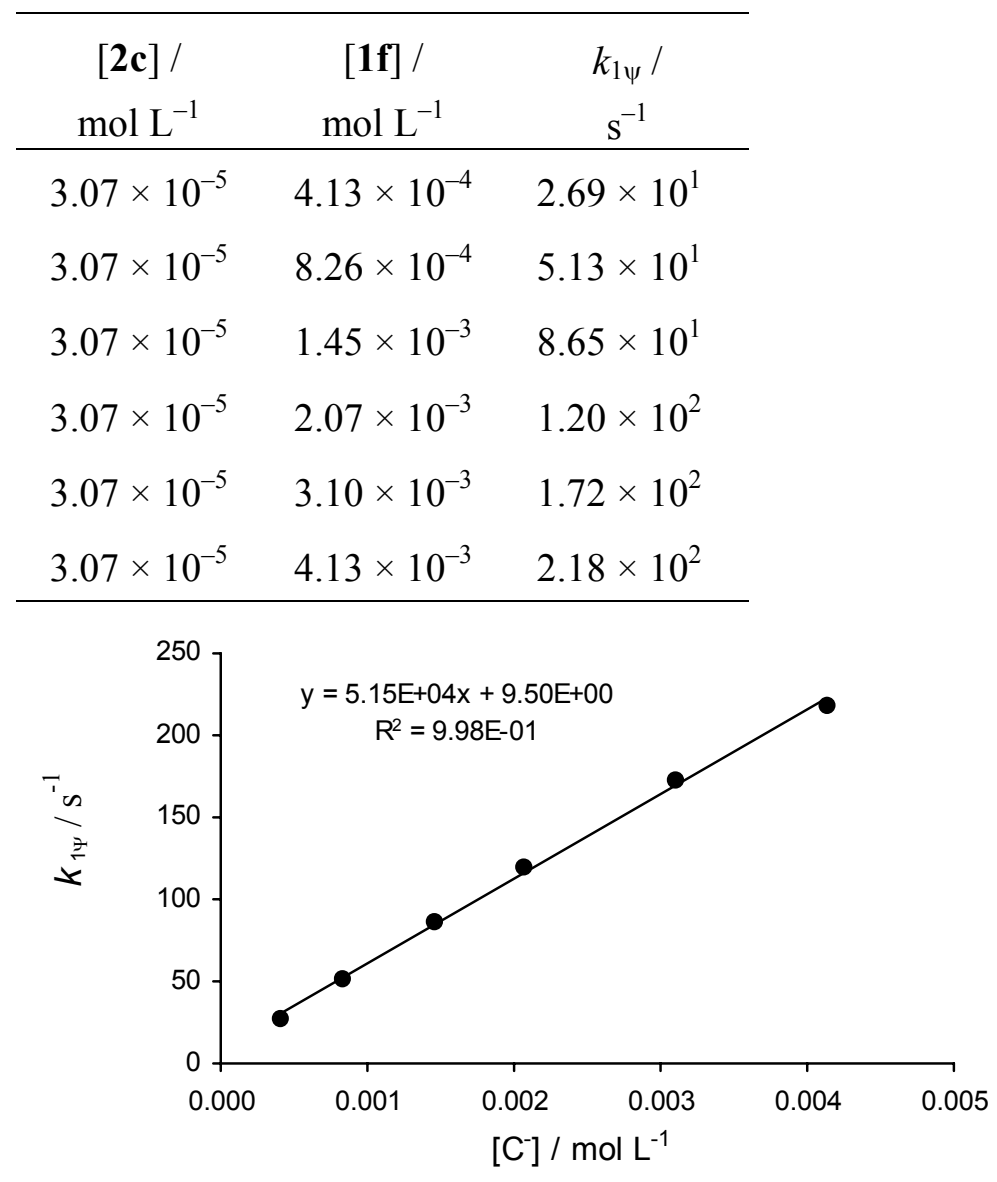

$k_{2, \mathrm{C}^{-}}=5.15 \times 10^{4} \mathrm{~L} \mathrm{~mol}^{-1} \mathrm{~s}^{-1}$ 
Table S43. (DBU)- $\mathrm{H}^{+}$salt of 4-nitrophenylnitromethanide (1f) and $\mathbf{2 f}$ in DMSO at $\lambda=371 \mathrm{~nm}$ at $20.0^{\circ} \mathrm{C}(\mathrm{J} \& \mathrm{M})$.

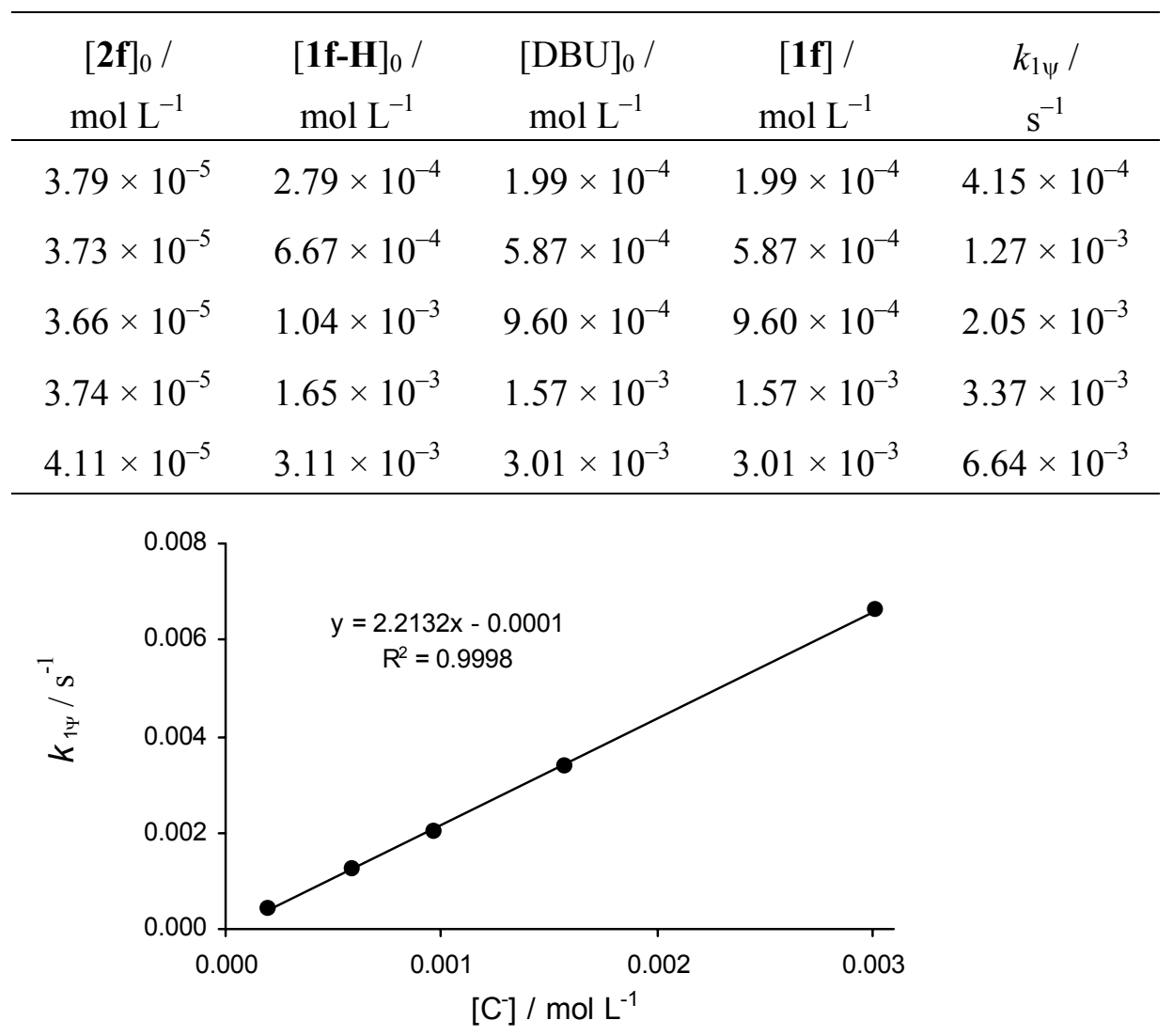

$k_{2, \mathrm{C}^{-}}=2.21 \mathrm{~L} \mathrm{~mol}^{-1} \mathrm{~s}^{-1}$ 
Table S44. (DBU)- $\mathrm{H}^{+}$salt of 3-nitrophenylnitromethanide (1g) and $2 \mathrm{c}$ in DMSO at $\lambda=640 \mathrm{~nm}$ at $20.0^{\circ} \mathrm{C}$ (stopped-flow).

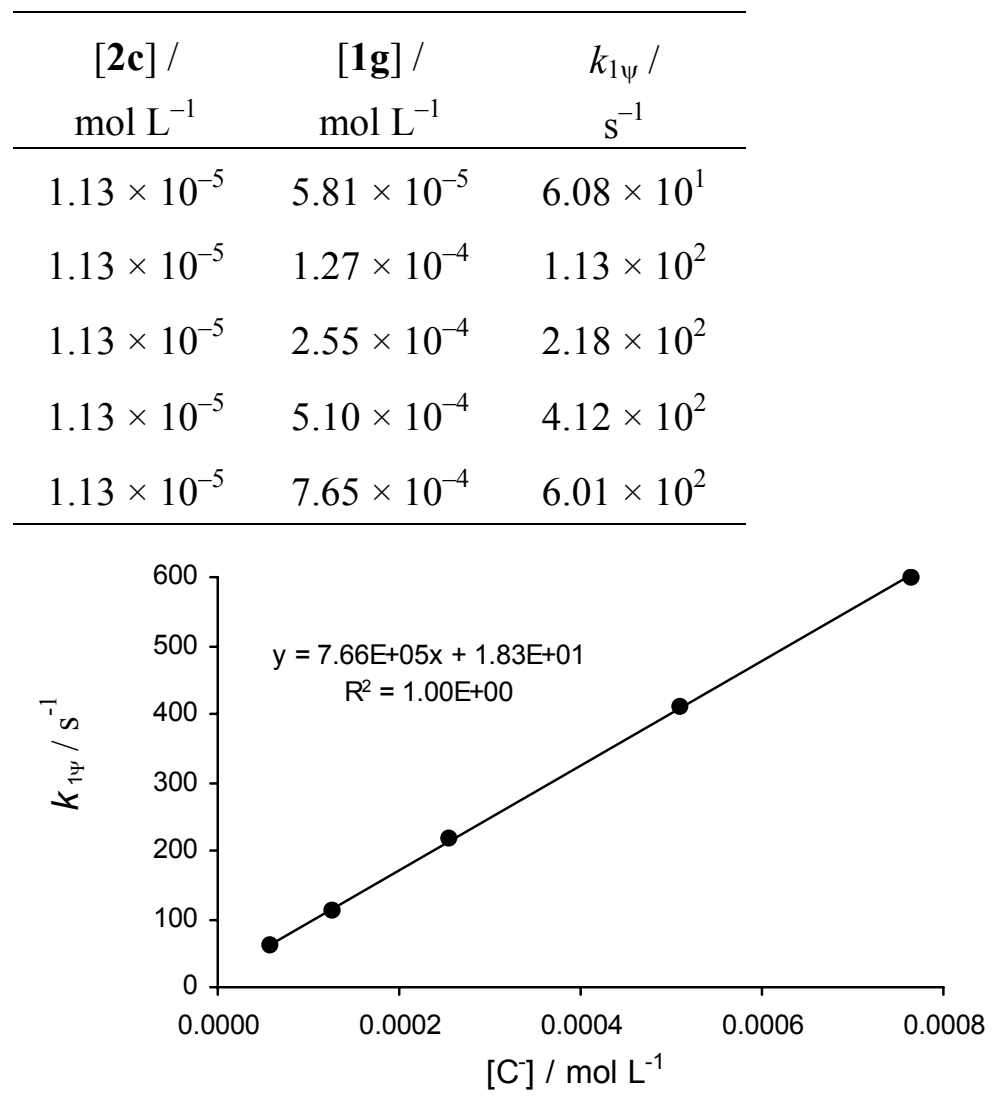

$k_{2, \mathrm{C}^{-}}=7.66 \times 10^{5} \mathrm{~L} \mathrm{~mol}^{-1} \mathrm{~s}^{-1}$ 
Table S45. (DBU)- $\mathrm{H}^{+}$salt of 3-nitrophenylnitromethanide (1g) and $2 \mathbf{e}$ in DMSO at $\lambda=533 \mathrm{~nm}$ at $20.0^{\circ} \mathrm{C}$ (stopped-flow).

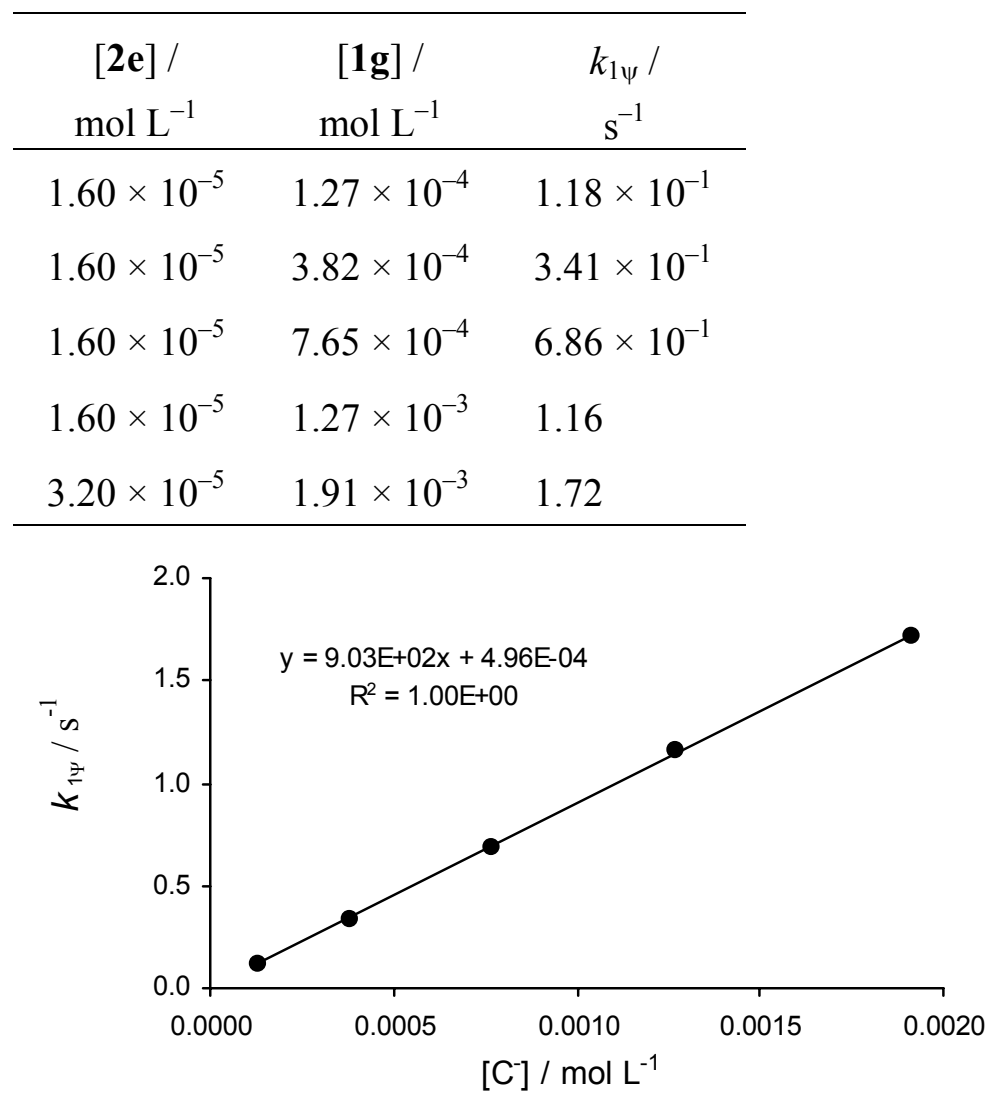

$k_{2, \mathrm{C}^{-}}=9.03 \times 10^{2} \mathrm{~L} \mathrm{~mol}^{-1} \mathrm{~s}^{-1}$ 
Table S46. (DBU)- $\mathrm{H}^{+}$salt of 3-nitrophenylnitromethanide (1g) and $2 \mathbf{i}$ in DMSO at $\lambda=521 \mathrm{~nm}$ at $20.0^{\circ} \mathrm{C}(\mathrm{J} \& \mathrm{M})$.

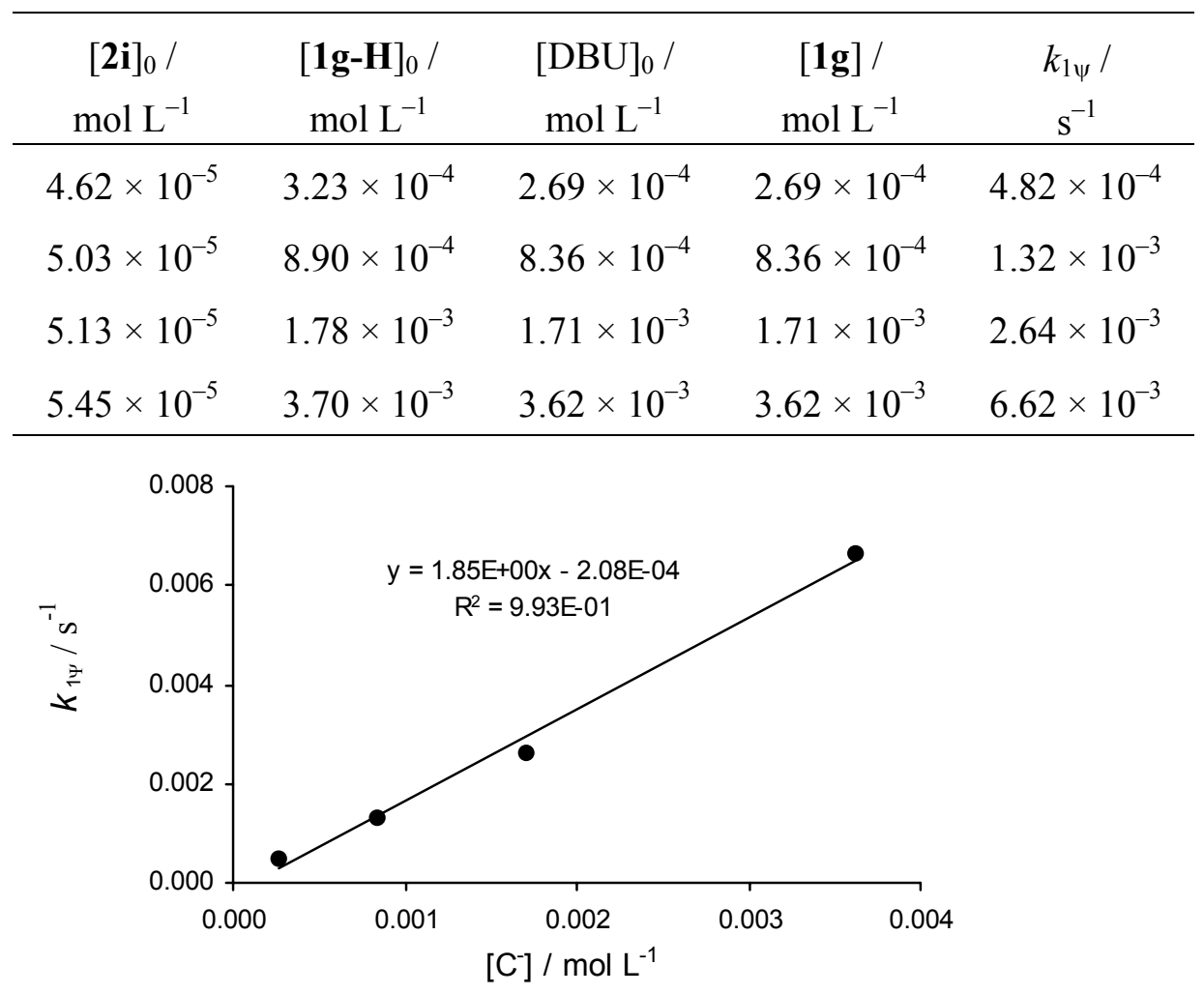

$k_{2, \mathrm{C}^{-}}=1.85 \mathrm{~L} \mathrm{~mol}^{-1} \mathrm{~s}^{-1}$ 
Table S47. (DBU)- $\mathrm{H}^{+}$salt of 4-cyanophenylnitromethanide (1h) and $\mathbf{2 c}$ in DMSO at $\lambda=640 \mathrm{~nm}$ at $20.0{ }^{\circ} \mathrm{C}$ (stopped-flow).

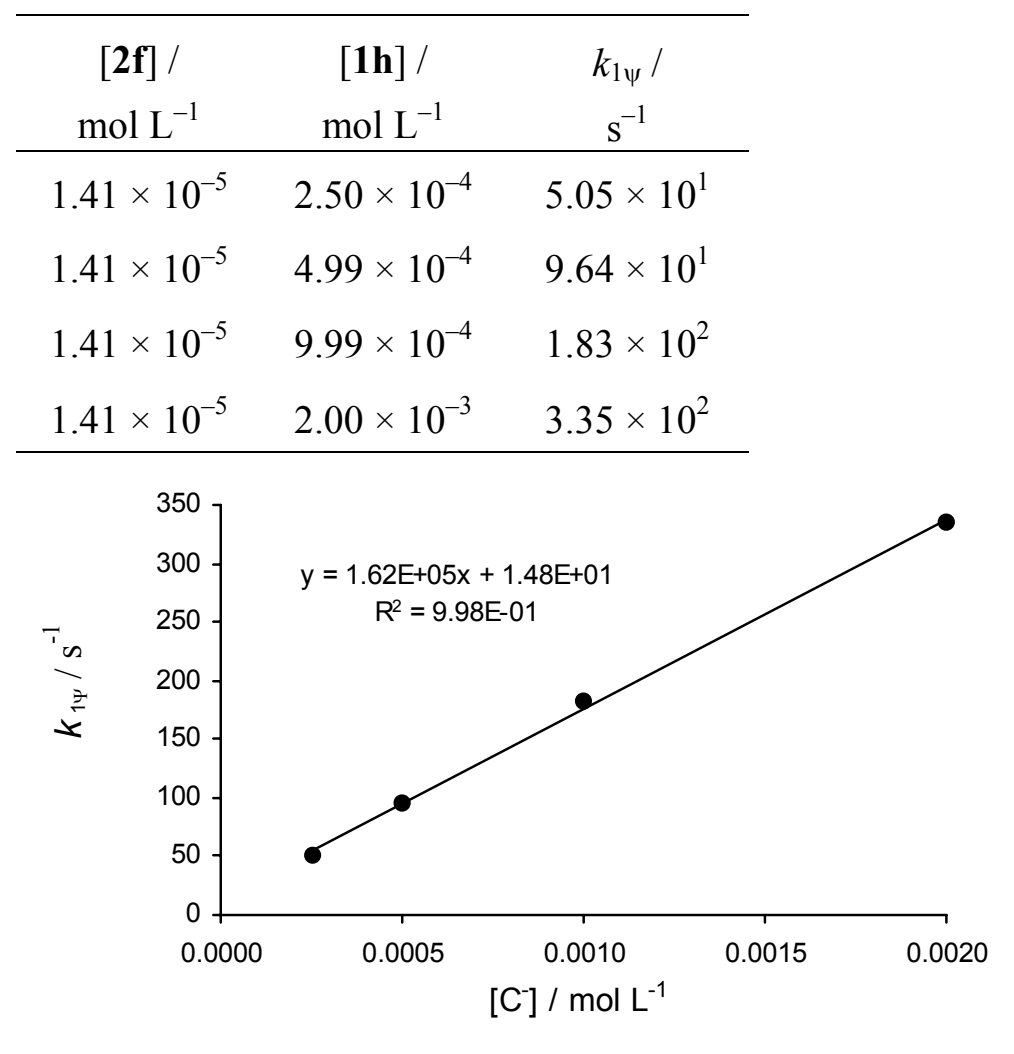

$k_{2, \mathrm{C}^{-}}=1.62 \times 10^{5} \mathrm{~L} \mathrm{~mol}^{-1} \mathrm{~s}^{-1}$ 
Table S48. (DBU)- $\mathrm{H}^{+}$salt of 4-cyanophenylnitromethanide (1h) and 2e in DMSO at $\lambda=533 \mathrm{~nm}$ at $20.0{ }^{\circ} \mathrm{C}$ (stopped-flow).

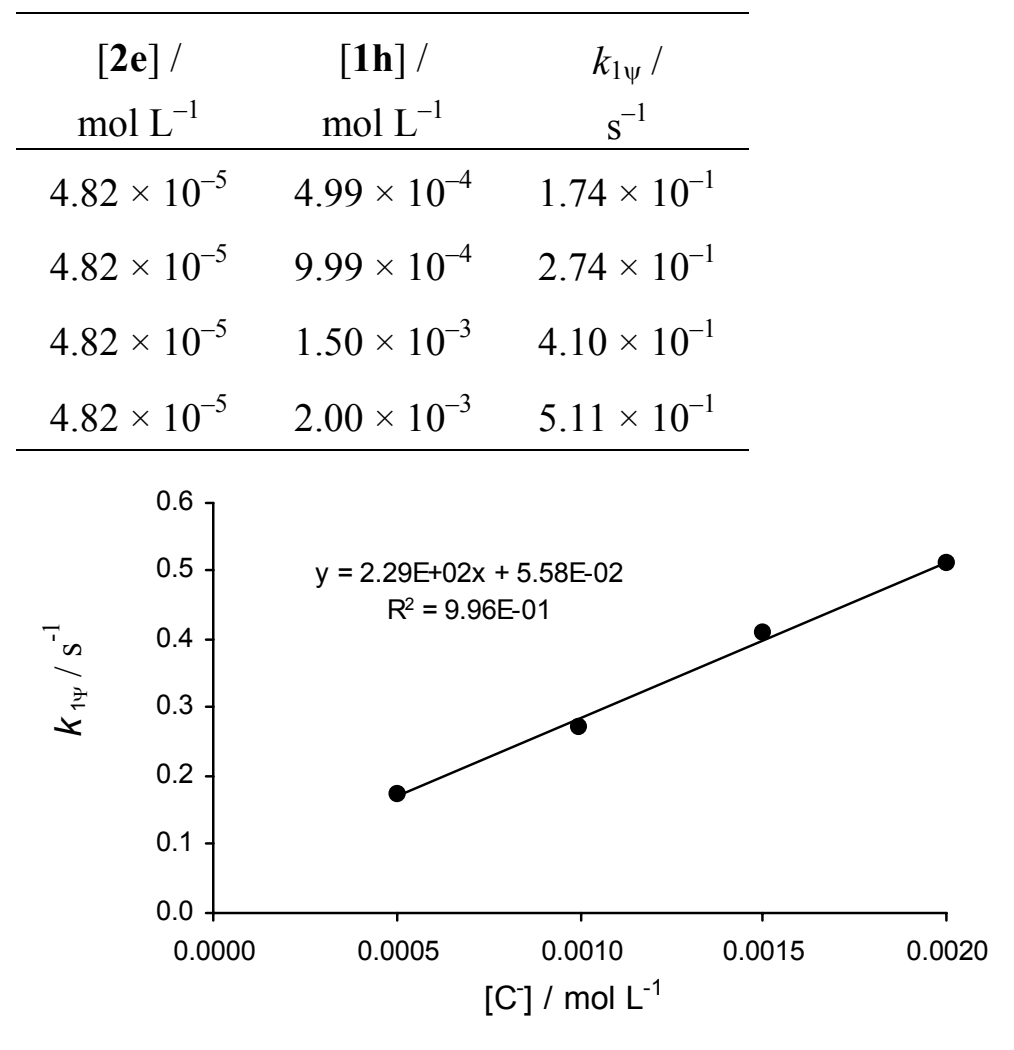

$k_{2, \mathrm{C}^{-}}=2.29 \times 10^{2} \mathrm{~L} \mathrm{~mol}^{-1} \mathrm{~s}^{-1}$ 
Table S49. (DBU)- $\mathrm{H}^{+}$salt of 4-cyanophenylnitromethanide (1h) and $\mathbf{2 h}$ in DMSO at $\lambda=488 \mathrm{~nm}$ at $20.0{ }^{\circ} \mathrm{C}(\mathrm{J} \& \mathrm{M})$.

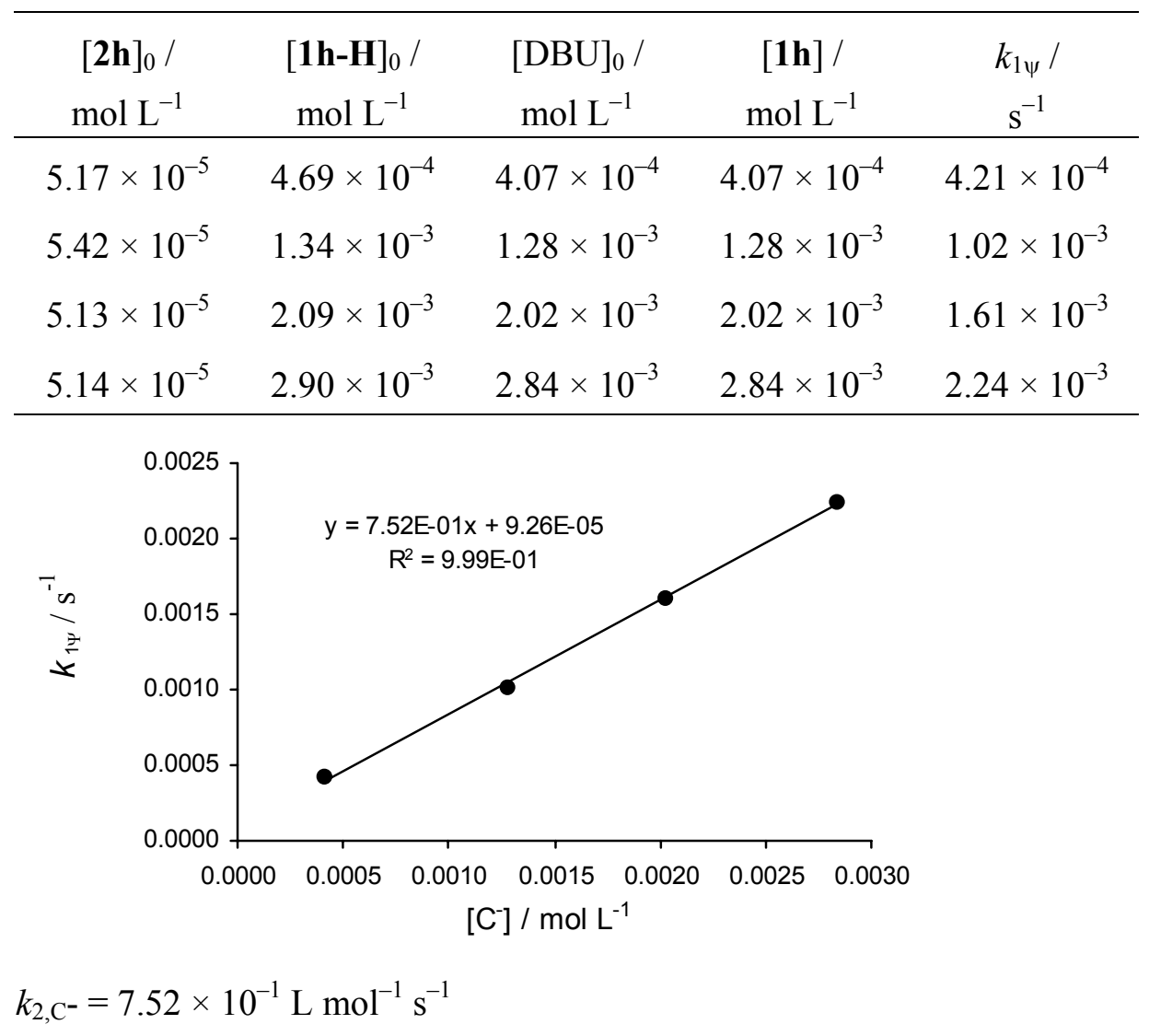




\section{References}

(S1) Kornblum, N.; Smiley, R. A.; Blackwood, R. K.; Iffland, D. C. J. Am. Chem. Soc. 1955, 77, $6269-6280$.

(S2) Hauser, F. M.; Baghdanov, V. M. J. Org. Chem. 1988, 53, 2872-2873.

(S3) Abell, A. D.; Hartshorn, M. P.; Maclennan, M. E.; Robinson, W. T.; Wright, G. J. Aust. J. Chem. 1991, 44, 43-51.

(S4) Suzuki, H.; Murashima, T.; Kozai, I.; Mori, T. J. Chem. Soc. Perkin Trans. 1 1993, 14, $1591-1597$. 